\title{
Functional Renormalization Group for Non-Equilibrium Quantum Many-Body Problems
}

\author{
Dissertation \\ zur Erlangung des Doktorgrades der Matematisch-Naturwissenschafftlichen \\ Fakultäten der Georg-August-Universität zu Göttingen
}

\author{
vorgelegt von \\ Riccardo Gezzi \\ aus Rom
}

angefertigt am

Institut für Theoretische Physik der

Georg-August-Universität zu Göttingen 2007 
Referent: Prof. Dr. Thomas Pruschke

Korreferent: Prof. Dr. Reiner Kree

Tag der mündlichen Prüfung: 


\section{Contents}

1 Introduction 5



1.2 Experimental Aspects . . . . . . . . . . . . . . . 5

1.2.1 Introduction and Fabrication Technique . . . . . . . . . 5

1.3 Two Parameter Regimes . . . . . . . . . . . . . . . . . 8

1.3.1 Single Electron Tunneling and Coulomb Blockade . . . . . . . 8

1.3.2 Kondo Regime . . . . . . . . . . . . . . . . . 10

1.4 Theoretical Interpretation . . . . . . . . . . . . . . . . . . . . . 12

1.4.1 Quantum Dots and Magnetic Impurities in Metals . . . . . . . 12

1.4.2 Transport due to the Kondo Effect . . . . . . . . . . . . . 12

1.5 Guide to this Work . . . . . . . . . . . . . . . . . . 15

2 The Keldysh Method 17

2.1 Introduction . . . . . . . . . . . . . . . . . . . . . . 17

2.2 Greens Functions in Non-Equilibrium . . . . . . . . . . . . . . . 17

2.3 Greens Functions for the Ideal Fermi Gas . . . . . . . . . . . . . . . . 19

2.4 Perturbative Expansion . . . . . . . . . . . . . . . . . . . 21

2.5 A useful Transformation . . . . . . . . . . . . . . . 25

3 Functional Renormalization Group $\quad 29$

3.1 Introduction . . . . . . . . . . . . . . . . . . . . . . . . . 29

3.2 Extension to Non-Equilibrium _. . . . . . . . . . . . . 30

3.3 The Flow Equations . . . . . . . . . . . . . . . . . . 33

3.4 Flow Equation for the Two-Particles Vertex . . . . . . . . . . . . 38

4 Stationary Transport $\quad 41$

4.1 Introduction . . . . . . . . . . . . . . . . . . . . 41

4.2 The Non-Interacting Case . . . . . . . . . . . . . . . . . . . . . . . . . . . . 41

4.3 Flow equations . . . . . . . . . . . . . . . . . . . 44

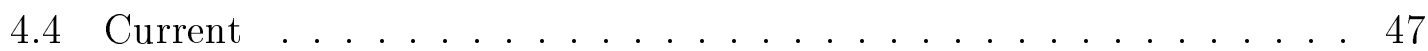

4.5 Lowest order approximation . . . . . . . . . . . . . . . . . . . . . . . . . . . . . . . . . 59

4.5.1 Non-equilibrium . . . . . . . . . . . . . . . . 51

4.6 Flowing vertex . . . . . . . . . . . . . . . . 54 
5 Transport Properties $\quad 63$

5.1 Introduction . . . . . . . . . . . . . . . . . 63

5.2 Flow Equations in a Magnetic Field . . . . . . . . . . . . . . . 63

5.3 Results . . . . . . . . . . . . . . . . . . 68

5.3.1 Equilibrium Case . . . . . . . . . . . . . . . . 68

5.3 .2 Non-Equilibrium . . . . . . . . . . . . . . . . . . . 70

5.3.3 Current and Conductance as Function of the Applied Bias . . 70

6 Temperature Dependence $\quad \mathbf{7 5}$

6.1 Introduction . . . . . . . . . . . . . . . . . . . . . . 75

6.2 Temperature Dependence of the Selfenergies . . . . . . . . . . . . 75

6.3 Results . . . . . . . . . . . . . . . . . . 77

6.3.1 Equilibrium . . . . . . . . . . . . . . . . 77

6.3.2 Current and Conductance as Function of the Applied Voltage 78

6.3 .3 Conductance as Function of $B \ldots \ldots 81$

7 Summary and Outlook $\quad 85$

8 Appendices $\quad 89$

8.1 The Morris Lemma . . . . . . . . . . . . . . . . . . . . . 89

8.2 Expressions Involving the Single Scale Propagator . . . . . . . . . . . 90

8.2.1 More Complicated Products . . . . . . . . . . . . . . . . . 91

8.3 Derivation of Eq. (3.11) . . . . . . . . . . . . . . . . . . 91

$\begin{array}{ll}\text { Bibliography } & 95\end{array}$

9 Lebenslauf 101 


\section{Introduction}

\subsection{An Overview}

The aim of this thesis is the extension of the functional renormalization group (FRG) formalism $[64,75,76,87]$ to treat non-equilibrium situations or, in other words, we reformulate the FRG-equations in terms of the Keldysh method [45] which is the standard technique to treat systems out of equilibrium. As simplest non-trivial application to test the potential and weakness of the non-equilibrium FRG we choose the single impurity Anderson model (SIAM) [4]. This model represents the paradigm for correlation effects in condensed matter physics and it is at the heart of a large range of experimental and theoretical investigations. In particular, the SIAM can be considered as the standard model for describing the physical properties of certain nanostructures and mesoscopic systems, such as quantum dots. A quantum dot can be viewd as a small region (in the next section we will precise what "small" means) consisting of semiconductor material, where electrons are confined by e.g. electrostatic potentials, by means of electron beam lithography and molecular beam epitaxy. The next paragraphs are dedicated to a short introduction regarding the experimental scenario and the theoretical interpretation of the characteristics of quantum dots. At the end of the present chapter we give the reader a "roadmap" which will guide him through this thesis.

\subsection{Experimental Aspects}

\subsubsection{Introduction and Fabrication Technique}

A quantum dot is an artificially structured system where the motion of particles is confined in all three spatial dimension, that can be filled with electrons (or holes). The dot can be coupled via tunnel barriers to reservoirs, with which electrons can be exchanged (see Fig. 1.1). By attaching current and voltage probes to the reservoirs, we can measure the electronic properties. The dot is also coupled capacitively to one or several gate electrodes, which can be used to tune the electrostatic potential of the dot with respect to the reservoirs. The previous description shows that a quantum dot is a rather general device, there consequently exist many different realizations: For instance single molecules trapped between electrodes [67], normal metal [68], superconducting [85], semiconductor lateral [49] or vertical dots 

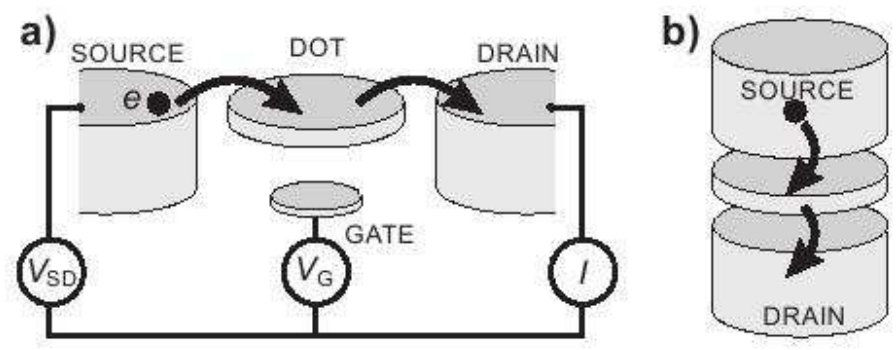

Figure 1.1: Schematic picture of a quantum dot in (a) a lateral geometry and (b) in a vertical geometry. The quantum dot is connected to source and drain reservoirs via tunnel barriers, allowing the current through the device, to be measured in response to a bias voltage, $V_{S D}$ and a gate voltage, $V_{G}$.

[51]. The electronic properties of quantum dots are dominated by two effects. First, the Coulomb repulsion between the electrons on the dot leads to an energy cost for adding an extra electron to the dot. Due to this charging energy, tunneling of electrons to or from the reservoirs can be dramatically suppressed at low temperatures; this phenomena is called Coulomb blockade [5]. Second, the confinement in all three dimensions leads to quantum effects that strongly influence the electron dynamics. One particularly frequent and well reproduced realization starts from heterostructures of GaAs and AlGaAs grown by molecular beam epitaxy (see Fig. 1.1). By doping the AlGaAs layer with $\mathrm{Si}$, free electrons are introduced. These accumulate at the $\mathrm{GaAs} / \mathrm{AlGaAs}$ interface, typically $50-100 \mathrm{~nm}$ below the surface, forming a two-dimensional electron gas (2DEG), a thin (approx. 10nm) sheet of electrons that can only move along the interface. The 2DEG can have a high mobility and relatively low electron density (typically $10^{5}-10^{7} \mathrm{~cm}^{2} / \mathrm{Vs}$ and $1-5 * 10^{15} / \mathrm{m}^{2}$, respectively. The low electron density results in a large Fermi wavelength (approx. 40 $\mathrm{nm}$ ) and a large screening length, which allows to locally deplete the 2DEG with an electric field. This electric field is created by applying a negative to metal gate electrodes on top of the heterostructure (see Fig. 1.1 (a)). Electron-beam lithography enables fabrication of gate structures with dimensions down to a few tens of nanometers (Fig. 1.1), yielding local control over the depletion of the 2DEG with roughly the same spatial resolution. Small islands of electrons can be isolated from the rest of the 2DEG by choosing a suitable design of the gate structure, thus creating quantum dots. Finally, low-resistance (Ohmic) contacts are made to the 2DEG reservoirs. To access the quantum phenomena in GaAs gated quantum dots, they have to be cooled down to $10-100 \mathrm{mK}$. 


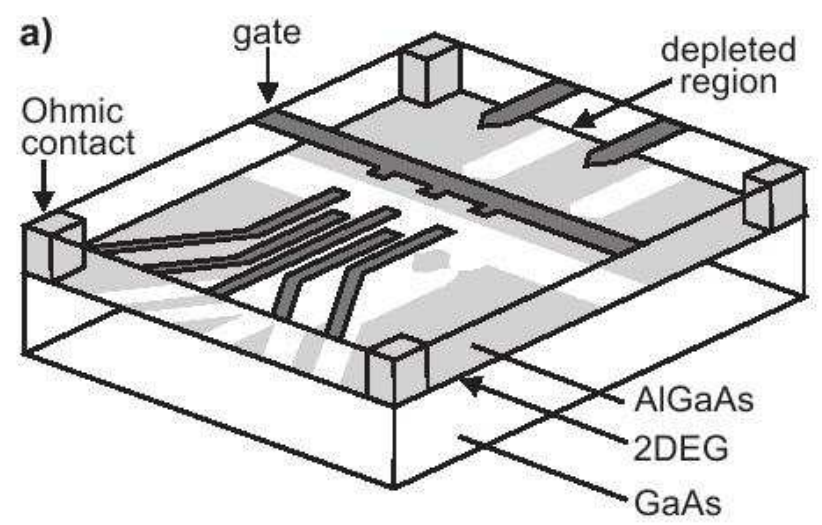

b)

c)
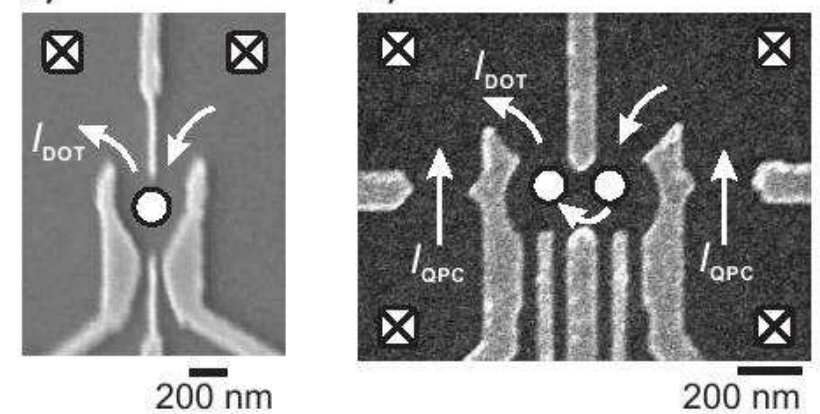

Figure 1.2: Lateral quantum dot device defined by metal surface electrodes. (a) Schematic view. Negative voltages applied to metal gate electrodes (dark gray) lead to depleted regions (white) in the 2DEG (light gray). Ohmic contacts (light gray columns) enable bonding wires (not shown) to make electrical contact to the 2DEG reservoirs. (b)-(c) Scanning electron micrographs of a few-electron single-dot device (b) and a double-dot device (c), showing the gate electrodes (light gray) on top of the surface (dark gray). The white dots indicate the location of the quantum dots. Ohmic contacts are shown in the corners. White arrows outline the path of current $J_{D O T}$ from one reservoir through the $\operatorname{dot}(\mathrm{s})$ to the other reservoir. For the device in (c), the two gates on the side can be used to create two quantum point contacts, which can serve as elec trometers by passing a current $J_{Q P C}$. Note that this device can also be used to define a single dot. 


\subsection{Two Parameter Regimes: Coulomb Blockade and Kondo}

\subsubsection{Single Electron Tunneling and Coulomb Blockade}

As we have already mentioned in the previous paragraph, experimental techniques like electron beam lithography and molecular beam epitaxy, permitted the realization of mesoscopic structure where is possible to confine a small amount of electrons in a spatial region of a few nanometers. One of the most interesting discovery, in these mesoscopic systems, was the single electron tunneling (SET) $([17,23])$, namely the possibility to let flow, in a controlled way, through the quantum dot one electron at a time. The scope of this section is to explain in which parameter range one can observe single electron tunneling and from which experimental quantity can be deduced that SET is indeed happening. In the linear regime the transport properties of mesoscopic systems can be represented by the conductance $G$ defined as

$$
G=\lim _{\left(V_{L}-V_{R}\right) \rightarrow 0}\left[\frac{J}{V_{L}-V_{R}}\right],
$$

where $J$ is the current flowing from the left to the right electrode and $V_{L, R}$ are the Fermi electrochemical potentials $\mu_{L, R}$ divided by the electronic charge. Before proceeding further we have to distinguish two parameter regimes. First we look at the limit in which the dot-leads coupling energy $\Gamma$ is small compared to the Coulomb energy $U$ on the island (Coulomb-blockade regime, Fig. 1.3 and Fig. 1.3.2 (c)) and then the case where $\Gamma$ cannot be neglected any more (see the next paragraph and Fig. $1.5(\mathrm{a})-(\mathrm{b}))$.

The experimental evidence of the single electron tunneling $[43,80]$ has been revealed for the first time by the periodic behaviour of the linear conductance $G$ as function of the gate potential $V_{G}$ (see Fig. 1.3). The peaks in Fig. 1.3 correspond to the flow of an electron from the left lead to the right one through the dot. The model explaining such a behaviour is usually called Coulomb blockade [43, 90]. It is based on simple electrostatic consideration we are going to elucidate. If we have electronic charge $Q$ on the island its energy is

$$
E=\frac{Q^{2}}{2 C_{T}}
$$

where $C_{T}$ represents the total capacitance between of dot. Now, if the potential drop between the gate and the island is $V_{G}$, the total electrostatic energy of the dot is given by

$$
E=-Q V_{G}+\frac{Q^{2}}{2 C_{T}}
$$

The first terms indicates the potential energy between the gate terminal (positively charged) and the central region, while the second takes into account the repulsive 

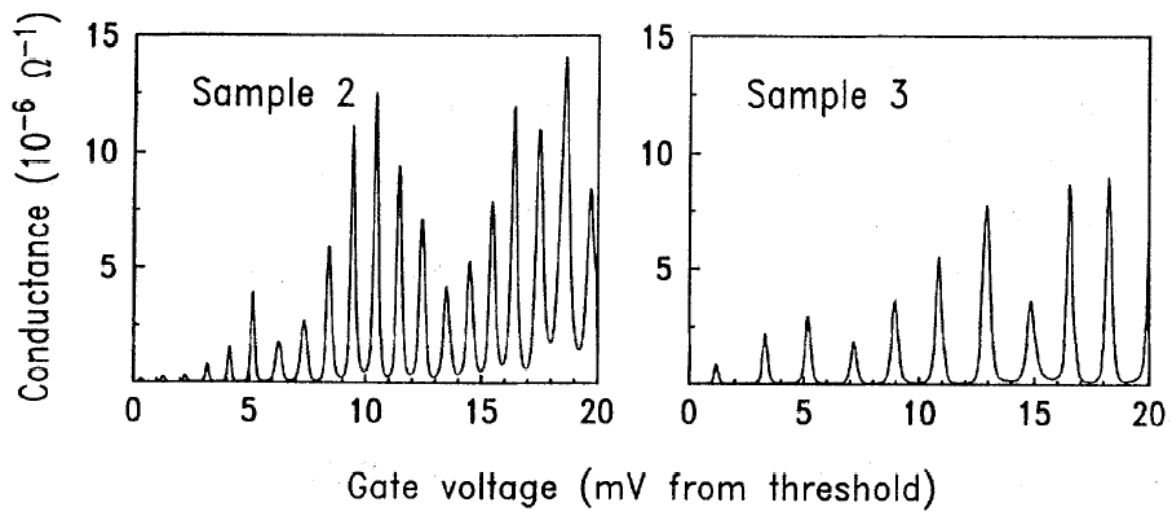

Figure 1.3: Conductance $G$ as function of the gate voltage $V_{G}$ in the parameter regime (Coulomb blockade) where the leads-dot coupling energy $\Gamma$ is negligible compared to the Coulomb repulsion $U$.

interaction among the charges on the dot. Equation (1.1) can be reexpressed (apart from a constant term) as

$$
E=\frac{\left(Q-Q_{0}\right)^{2}}{2 C_{T}}
$$

with $Q_{0}=C_{T} V_{G}$. For any given value of $V_{G}$ (and thus of $Q_{0}$ ), the charge on the dot adjustes itself to minimize the energy. Neverthless, because of the charge quantization $Q=N e$, for a given $Q_{0}$, we can only have discrete energy levels (see Fig. 1.4). As
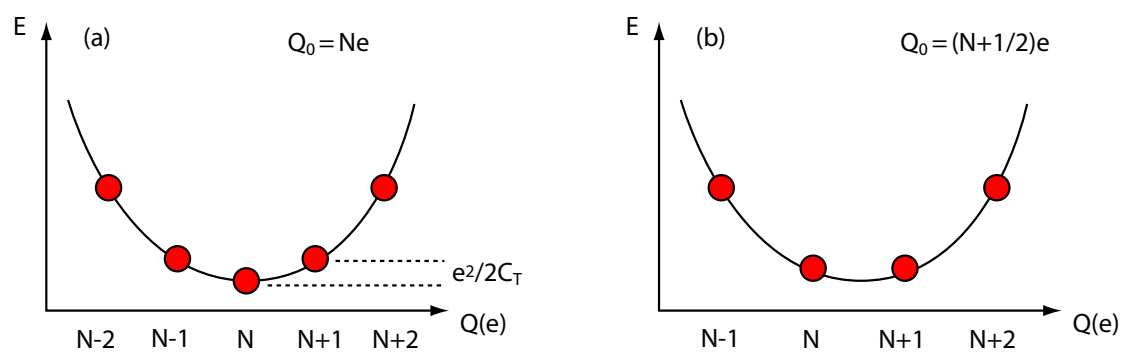

Figure 1.4: Total energy of a quantum dot plotted as function of the elementar electronic charge. (a) Shows the case with a non-degenerate energetic minimum: $Q_{0}=N e$, (b) degenerate: $Q_{0}=(N+1 / 2) e$.

soon as $Q_{0}=N e$, the energy is minimized by an integer number of electrons and the increase or the decrease of the charge $\pm e$ costs an activation energy corresponding to (see Fig. 1.4 (a))

$$
E_{A}=\frac{e^{2}}{2 C_{T}}
$$


On the other hand, if $Q_{0}=\left(N+\frac{1}{2}\right) e$, then the states characterized by $Q=N e$ and $Q=(N+1) e$ are degenerate (see Fig. $1.4(\mathrm{~b}))$ so that the charge fluctuates between these two values, even at zero temperature, without any energetic expense. From the experimental point of view this means that the conductance is different from zero for all value of $V_{G}$ with

$$
V_{G}=Q_{0} / C_{T}=\left(N+\frac{1}{2}\right) e / C_{T}
$$

For all other values of $V_{G}$ the conductance is very small or zero. This is the reason why, at low temperatures, we observe periodically spaced conductance peaks. The period is determined by $V_{G}=\frac{e}{C_{T}}$ and corresponds to the variation of $V_{G}$ which is necessary to let $Q_{0}$ pass from $\left(N+\frac{1}{2}\right) e$ to $\left(N+\frac{3}{2}\right) e$.

\subsubsection{Kondo Regime}

Consider now the situation in which $\Gamma$ the coupling parameter between dot and leads is not negligible any longer (Kondo regime) [11, 22]). In this limit $G$ as function of the gate voltage presents different features (see Fig. 1.3.2 (a) (b)) compared to the Coulomb blockade regime shown in Fig. 1.3 and in Fig. 1.3.2 (c). The most characteristic aspect is that the peaks now form pairs. Peaks belonging to the same pair show a comparable width (see Fig.1.3.2 (a) (b)) and their spacing is determined by the Coulomb repulsion $U=\frac{e^{2}}{2 C_{T}}$. On the other hand, between different pairs we observe a spacing greater then $U$ and different peak widths.

This behaviour can be explained assuming that in an electronic state we cannot insert more then two electrons with opposite spin. In fact, if we wish to add a third electron, we are forced to insert it in a state which is different from the one occupied by the previous pair. As direct consequence the spacing between peak pairs takes into account both $U$ and the energy difference between the two successive energetic levels $\Delta \varepsilon[11,22])$, resulting in a total spacing given by $U+\Delta \varepsilon$. If we look at the valleys in Fig. 1.3.2 (a) (b), we find that the intra-pair ones show a higher conductance compared to the inter-pair valleys. The reason for this behaviour lies in the number of electrons present on the island. The valley between two paired peaks corresponds to a dot occupied with an odd number of electrons, therefore the unpaired electron can interact with the charges at the Fermi level in the leads, giving rise to a Kondo singlet bound state between them (see next paragraph). The singlet increases the dot density of state (DOS) at the Fermi level (see next paragraphs and Fig. 1.8 ), which increases the conductance too. If we lower the temperature $T$ the peaks tend to become narrower and higher (even in the limit in which $\Gamma$ is not small), neverthless the intra-pair conductance valley increases while the interpair one decreases, letting the conductance become small. We observe exactly the opposite phenomenon increasing the temperature, because an increase of $T$ tends 




Figure 1.5: Temperature dependence of zero-bias conductance $G$ through two different spatial states on the dot. (a), Paired peaks corresponding to the two spin states for each spatial state become better resolved with increasing temperature from $90 \mathrm{mK}$ (full line) to $400 \mathrm{mK}$ (dashed). The intra-pair valleys become deeper and the peaks become narrower. (b), From $400 \mathrm{mK}$ (dashed line) to $800 \mathrm{mK}$ (dotted) the paired peaks near $V_{G}=-70 \mathrm{mV}$ broaden. The peaks near $V_{G}=-25 \mathrm{mV}$ are still becoming better resolved even at 800 $\mathrm{mK}$, as they have larger $\Gamma$. (c), When $\Gamma$ is reduced (as illustrated by shorter and narrower peaks), $U$ increases relative to $\Delta \varepsilon$, so peak pairing is no longer evident. Because the Kondo phenomenon is suppressed, peaks become narrower as temperature is decreased at all $T$ down to our base temperature of $90 \mathrm{mK}$. Full line is for $90 \mathrm{mK}$, dotted line for $800 \mathrm{mK}$. 
to destroy the spin correlations between the electrons of the central region and the leads, letting the conductance relative to the intra-pair valley collapse.

\subsection{Theoretical Interpretation}

\subsubsection{Quantum Dots and Magnetic Impurities in Metals}

Glazman and Raikh [21] noted that quantum dots can be related to metals doped with magnetic impurities in such a way that the dot, occupied by just one electron, plays the role of the impurity and the electrodes represent the metal matrix in which the impurity is embedded.

They understood that, even if electronic transport is in principle forbidden by the Coulomb blockade, it can still occur by means of the Kondo effect [47]. This idea has been verified experimentally later by Ralph and Buhrman [71] and then by Goldhaber-Gordon [22] and Cronenwett et al. [11], [34]. A detailed discussion of the Kondo effect is far beyond the scope of this work (the interested reader is referred to the book by A.Hewson [34]), here we just give some basic introduction on the Kondo Physics.

The original problem stems from the anomalous behaviour of the resistivity $\rho$ as function of temperature $T$ in metal doped with magnetic impurities. Experimentally it was observed that the the resistivity of such metals does not simply decrease, when the temperature is lowered, but it shows a minimum and then it begins to grow again before it saturates [24, 34, 59]. The explanation of this behaviour can be found in the spin-flip scattering between the spins of the conduction electrons and the magnetic impurity. The latter can be seen as a scattering center whose interaction with the conduction electrons causes the spin flip scattering. This kind of interaction appears when the temperature is low enough to suppress thermal fluctuations, a direct consequence is the anomalous resistivity behaviour.

Below a characteristic temperature, also called Kondo temperature $T_{K}$, the spin of the impurity forms a many-body state with the band electrons. This means that if we want to create a spin triplet between the host and the electrons we must break up the many-body state or in other words we should give to the system an energy which is larger then the binding energy $T_{K}$ of this many-body state. Therefore the spin flip scattering, at $T<T_{K}$, is frozen out and the Kondo effect saturates.

\subsubsection{Transport due to the Kondo Effect}

The analogies between quantum dots and metals doped with magnetic impurities $[21,22,49,71]$ permit us to explain the charge transport through quantum dots in the Coulomb blockade regime by means of the Kondo effect. 
The standard approach to describe the phenomenology of quantum dots is based on the single impurity Anderson model SIAM [4], given by the Hamiltonian

$$
\begin{aligned}
H= & \sum_{\vec{k} \sigma \alpha} \varepsilon_{\vec{k} \sigma \alpha} c_{\vec{k} \sigma \alpha}^{\dagger} c_{\vec{k} \sigma \alpha} \\
& +\sum_{\sigma} \varepsilon_{\sigma} d_{\sigma}^{\dagger} d_{\sigma}+U\left(n_{\uparrow}-\frac{1}{2}\right)\left(n_{\downarrow}-\frac{1}{2}\right) \\
& +\sum_{\vec{k} \sigma \alpha}\left[V_{\vec{k} \sigma \alpha} c_{\vec{k} \sigma \alpha}^{\dagger} d_{\sigma}+\text { h.c. }\right]
\end{aligned}
$$

where $c, d$ denote the operators for the conduction electrons and the impurity degrees of freedom and $n_{\sigma}=d_{\sigma}^{\dagger} d_{\sigma}$. $U$ represents the Coulomb repulsion on the impurity site, $V_{\vec{k} \sigma \alpha}$ is the hopping term which permits an electron of the leads to jump onto the dot and vice versa. Finally $\alpha=L, R$ distinguishes the left and right reservoir. The spin indices $\sigma$ can take the values up or down $(\uparrow, \downarrow)$ and the band wavevector $\vec{k}$ runs through all values of the first Brillouin zone of the leads.

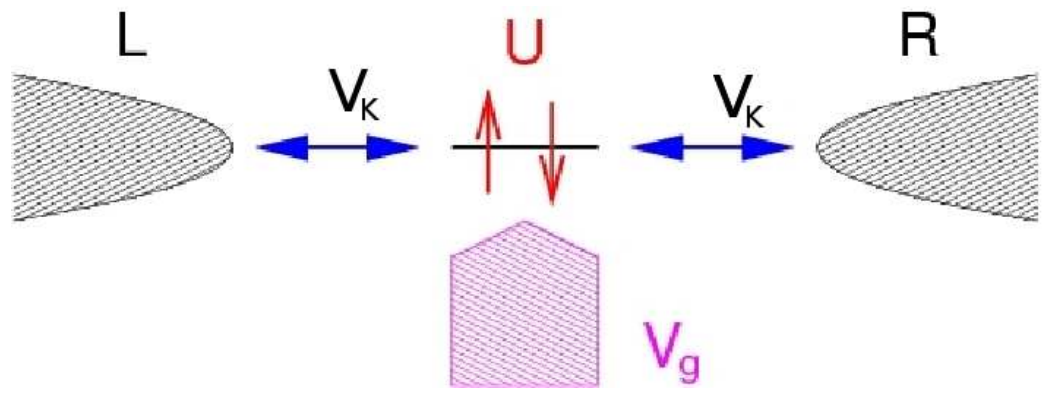

Figure 1.6: Pictorial representation of the impurity Anderson model: The dot in the central region, subject to the Coulomb repulsion $U$, is coupled via $V_{K}$ to the left and right leads which are here at the same electrochemical potential. An additional electrode $V_{g}$ controls capacitively the charge on the dot.

Looking at Fig. 1.7 (a) (b) charge transport from the left electrode to the right one (which are kept at a constant bias $V_{L}-V_{R}=\frac{\mu_{L}-\mu_{R}}{e}$ ) is permitted if the the dot level is located in the following energetic interval (see Fig. 1.6)

$$
\mu_{L}<\varepsilon_{d}<\mu_{R}
$$

Now assume that the temperature is much lower than the energetic spacing between two levels $|\Delta \varepsilon|$ of the central region, therefore it can be treated as if it consisted of just a single level. If we now let flow another electron on the dot then the level is pushed up by an energy

$$
\Delta \varepsilon=\varepsilon_{d}+U
$$


(a)
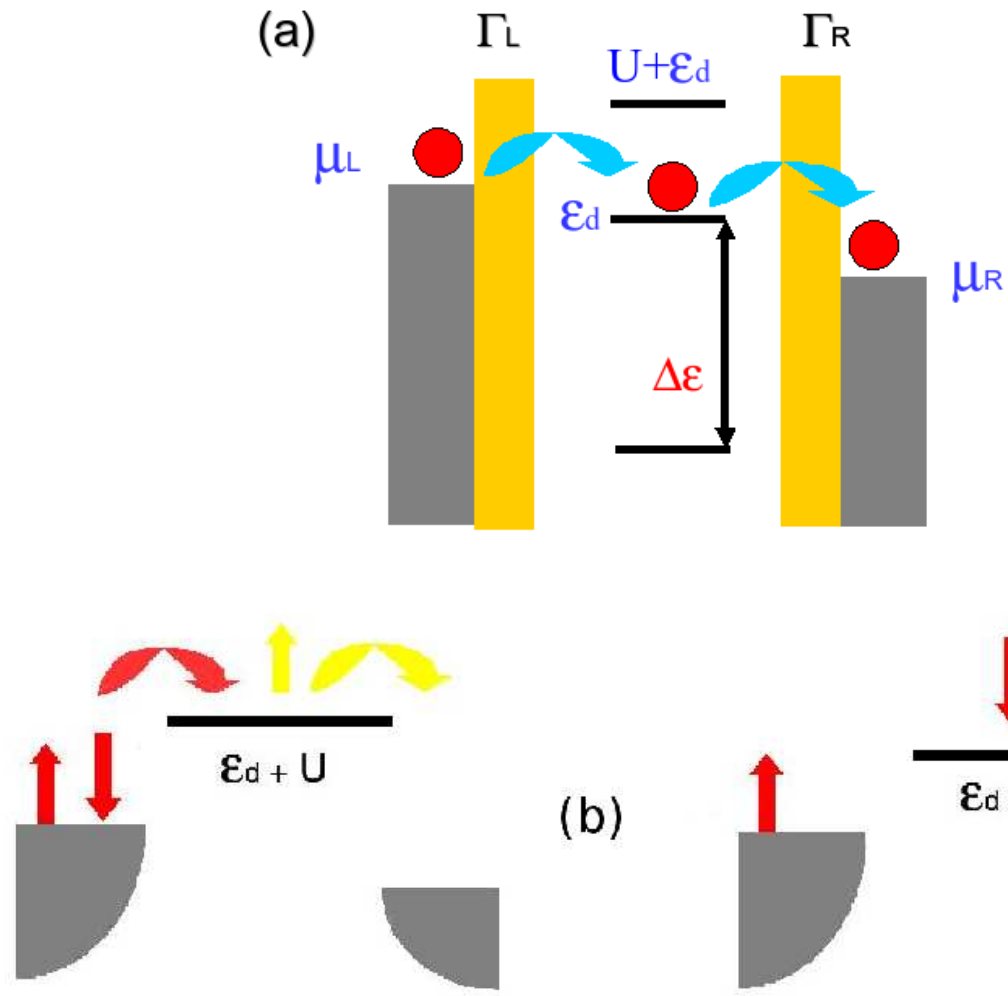

(b)

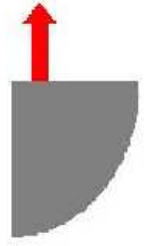

$\varepsilon_{\mathrm{d}+U}$

Figure 1.7: (a) Tunnel process through the quantum dot. Transport can take place if $\mu_{R}<\varepsilon_{d}<\mu_{L}$. If the temperature $T$ is low enough then the energetic spacing $\Delta \varepsilon$ is too large to permit an electron to jump from an energetic level to the next one $\rightarrow$ single-level quantum dot. (b) Pictorial description of the transport in the Coulomb blockade regime through the Kondo effect: virtual tunneling transition whose end effect is always a dot occupied by a single electron, but with opposite spin. 
Therefore the level is shifted above the energy interval previously defined, thus making transport impossible at first sight. In reality we can have virtual tunneling transitions due to the dot-leads coupling which can induce a spin flip between the central region (see Fig. 1.7 (b)) and the leads. Actually, these spin flip processes give rise to the formation of a singlet state between the electron on the island end the ones in the leads (Kondo effect $[11,22,34]$ ). The presence of such a singlet is connected to a scattering resonance at the Fermi level (see Fig. 1.8). Experimentally this additional spectral weight, due to manybody effects, manifests itself in an increase of the conductance between the Coulomb peaks, i.e. in the intra-pair valley region shown in Fig. 1.3.2 (a) (b).



Figure 1.8: Schematic energy diagram of the SET, showing an electron droplet separated by tunnel barriers from conducting leads. Since the number of electrons in the droplet is odd, the local density of states exhibits a sharp Kondo resonance at the Fermi level. The broad resonance at energy $\varepsilon_{0}$ represents a transition from $n_{d}=0 \rightarrow n_{d}=1$, while the one at $\varepsilon_{0}+U$ corresponds to a transition from $n_{d}=1 \rightarrow n_{d}=2$.

\subsection{Guide to this Work}

The present thesis is divided into six chapters and two appendices. In the next chapter we discuss in detail the Keldysh method, which is the standard technique for the description of non-equilibrium quantum manybody problems. The third chapter is dedicated to the renormalization group. It will focus on the extension of the functional renormalization group (FRG) to non-equilibrium situations. First of 
all we will show the differences between equilibrium and non-equilibrium and then that the FRG can be extended to treat, at least formally, also time dependent nonequilibrium situations and not only the stationary case (constant external field). In the last three chapters we will accompany the reader through our results, for the transport parameters, the current $J$ and conductance $G$, calculated with the non-equilibrium FRG.

We will concentrate on the simplest case, namely a situation where we neglect the energy dependence of the quantities entering into the FRG equations. We also discuss the influence of an applied magnetic field $B$, in order to distinguish the contributions of different spin channels to the transport parameters, and the temperature $T$. Summary and conclusion will finish this work. 


\section{The Keldysh Method}

\subsection{Introduction}

The physical properties of a system in equilibrium can be extracted from the Green function defined as $^{1}$

$$
G(1,2)=-i\left\langle\hat{T} \psi_{H}(1) \psi_{H}^{\dagger}(2)\right\rangle
$$

where $\psi_{H}(1)$ represents a field operator in the Heisenberg picture which annihilates an ingoing particle at point $x_{1}$ and at the time $t_{1}$, while $\psi_{H}^{\dagger}(2)$ creates an outgoing particle at $x_{2}, t_{2} . \hat{T}$ stands for the time order operator, which places the operators according to the time argument with the latest time to the left $[29,52,59]$. The mean value is calculated with respect to the Gibbs distribution function. From the statistical point of view it makes no no difference if we calculate the mean value of a system in equilibrium with respect to the exact wave function of the stationary state of the closed system or by means of the Gibbs distribution of the system in a thermal bath. In the first case the result will be a function of the energy and the number of particles and in the second of the temperature and the chemical potential. The situation changes as soon as we treat systems out of equilibrium. We have to calculate averages with respect to states which are not necessarely the ground state or even eigenstates of the system, but resulting from initial conditions determined by external fields, preparation, etc [40, 45, 54, 82]. As direct consequence (see Sections 2.2-2.4) we are obliged to introduce four Green functions, taking into account the excitation spectrum and the distribution function separately. Moreover, the correlation functions we are going to analyze contain only real variables. This permits us to avoid the analytic continuation (as necessary in the Matsubara technique), which can be a cumbersome task, in particular if results are known only numerically.

\subsection{Greens Functions in Non-Equilibrium}

Before explaining the mathematical details of the non-equilibrium perturbation theory, we first prefer to present the basic idea and functions on which it bases. In the next section we will then apply it to the easiest case, namely to the non interacting Fermi gas and finally in the Paragraph 2.4 we will explain the perturbative

\footnotetext{
${ }^{1}$ For brevity we restrict ourselves to fermions. The formula for bosons is identical except for a different sign.
} 
expansion. Let us start defining ${ }^{2}$

$$
G(1,2)=-i\left\langle T_{K} \psi_{H}(1) \psi_{H}^{\dagger}(2)\right\rangle
$$

The quantity $G(1,2)$ depends separately on the pair of variables $(1,2)$ and not only on their difference, as in equilibrium. Compared to the "usual" time ordering operator the one in the Eq. (2.2) carries an index $K$, which describes a path starting at $t=-\infty$ up to $t=+\infty$ and then back again in the opposite direction. Such a path is also called Keldysh contour (see Fig. 2.1) [45]. Starting from the definition



Figure 2.1: The Keldysh contour: Start the time evolution at time $t=-\infty$ up to $t=+\infty$ (blue line) and then back from $t=+\infty$ up to $t=-\infty$ (red line).

Eq. (2.2) we now displace the times $t_{1}, t_{2}$, on the Keldysh contour in all possible ways $[29,52]$. Since we have only two times to play with, there are just four possible combinations and to each one corresponds a certain Green function, which will be labeled by means of two upper indices, each of them representing the upper $(-)$ or the lower branch $(+)$ of the Keldysh contour. We obtain

$$
\begin{aligned}
G^{--}(1,2) & =-i\left\langle T \psi_{H}(1) \psi_{H}(2)^{\dagger}\right\rangle= \\
& =-i \theta\left(t_{1}-t_{2}\right)\left\langle\psi_{H}(1) \psi_{H}(2)^{\dagger}\right\rangle+i \theta\left(t_{2}-t_{1}\right)\left\langle\psi_{H}(2)^{\dagger} \psi_{H}^{\dagger}(1)\right\rangle
\end{aligned}
$$

where $t_{1}, t_{2} \in C_{K_{-}}$, (G time-ordered) and $C_{K_{-}}$is the upper branch of the contour;

$$
\begin{aligned}
G^{++}(1,2) & =-i\left\langle\tilde{T} \psi_{H}(1) \psi_{H}(2)^{\dagger}\right\rangle= \\
& =-i \theta\left(t_{2}-t_{1}\right)\left\langle\psi_{H}(1) \psi_{H}(2)^{\dagger}\right\rangle+i \theta\left(t_{1}-t_{2}\right)\left\langle\psi_{H}(2)^{\dagger} \psi_{H}(1)\right\rangle
\end{aligned}
$$

with both times on the lower branch of the contour $t_{1}, t_{2} \in C_{K_{+}}$(G antitimeordered);

$$
G^{+-}(1,2)=-i\left\langle\psi_{H}(1) \psi_{H}(2)^{\dagger}\right\rangle
$$

(G greater) with $t_{1} \in C_{K_{+}}, t_{2} \in C_{K_{-}}$, and finally

$$
G^{-+}(1,2)=i\left\langle\psi_{H}(2)^{\dagger} \psi_{H}(1)\right\rangle
$$

(G lesser) where we have $t_{1} \in C_{K_{-}}, t_{2} \in C_{K_{+}}$. The last two Green functions are not time-ordered and moreover couple the two branches of the contour. From the

\footnotetext{
${ }^{2}$ Here and in the following we set $\hbar=1$
} 
previous definitions it follows that Eqs. (2.3)-(2.6) are not independent, but have to obey the constraint

$$
G^{--}+G^{++}=G^{-+}+G^{+-} .
$$

One can introduce two more Green functions

$$
\begin{aligned}
G^{A}(1,2) & =i \theta\left(t_{2}-t_{1}\right)\left\langle\left\{\psi_{H}(1), \psi_{H}^{\dagger}(2)\right\}\right\rangle= \\
& =\theta\left(t_{2}-t_{1}\right)\left[G^{-+}(1,2)-G^{+-}(1,2)\right], \\
G^{R}(1,2) & =-i \theta\left(t_{1}-t_{2}\right)\left\langle\left\{\psi_{H}(1), \psi_{H}^{\dagger}(2)\right\}\right\rangle= \\
& =\theta\left(t_{1}-t_{2}\right)\left[G^{+-}(1,2)-G^{-+}(1,2)\right],
\end{aligned}
$$

the retarded and advanced Green functions defined exactly as in equilibrium. They can be written in terms of $G^{+-}, G^{-+}$according to

$$
\begin{aligned}
& G^{R}=G^{--}-G^{-+}=G^{+-}-G^{++}, \\
& G^{A}=G^{--}-G^{+-}=G^{-+}-G^{++} .
\end{aligned}
$$

From the definitions (2.3)-(2.4) follows, because of the antihermicity of their arguments, that

$$
G^{--}(1,2)=-G^{++}(2,1)
$$

The lesser and greater functions, are antihermitic

$$
G^{-+}(1,2)=-\left[G^{-+}(2,1)\right]^{*}, G^{+-}(1,2)=-\left[G^{+-}(2,1)\right]^{*} .
$$

This means that their Fourier components are purely imaginary. Consider now the non-equilibrium stationary and space-homogeneous case. Thanks to the space and time transational invariance, all correlation functions, now again, depend only on the differences of their arguments $t=t_{1}-t_{2}, \mathbf{x}=\mathbf{x}_{1}-\mathbf{x}_{2}$. It is then possible to take the Fourier transform with respect to such differences, which directly leads to the relations

$$
\begin{gathered}
G^{--}(\omega, \mathbf{p})=-\left[G^{++}(\omega, \mathbf{p})\right]^{*}, \\
G^{A}(\omega, \mathbf{p})=\left[G^{R}(\omega, \mathbf{p})\right]^{*} .
\end{gathered}
$$

\subsection{Greens Functions for the Ideal Fermi Gas}

The physical meaning of the previously introduced Green functions can be illustrated through a simple example, namely the non interacting Fermi gas in its homogeneous and stationary state [52]. Start from the Eq. (2.5) in which we substitute for the $\psi$-operators the free-field expansion explicitely written in the variables $\mathbf{x}, t$ as

$$
\hat{\psi}_{H}(t, \mathbf{x})=\frac{1}{V^{\frac{1}{2}}} \sum_{\mathbf{p}} \hat{a}_{\mathbf{p}} e^{(i[\mathbf{p} \mathbf{x}-\varepsilon(\mathbf{p}) t+\mu t])},
$$




$$
\hat{\psi}_{H}^{\dagger}(t, \mathbf{x})=\frac{1}{V^{\frac{1}{2}}} \sum_{\mathbf{p}} \hat{a}_{\mathbf{p}}^{\dagger} e^{(-i[\mathbf{p x}-\varepsilon(\mathbf{p}) t+\mu t])},
$$

where $a_{\mathbf{p}}, a_{\mathbf{p}}^{\dagger}$ are the usual annihilation and creation operators, $\varepsilon(\mathbf{p})=\frac{\mathbf{p}^{2}}{2 m}$ and $\mu$ is the chemical potential. With this substitution we obtain

$$
\left\langle\psi(\mathbf{x}, t) \psi^{\dagger}\left(\mathbf{x}^{\prime}, t^{\prime}\right)\right\rangle=\frac{1}{V} \sum_{\mathbf{p}, \mathbf{p}^{\prime}} e^{\left\{i\left[\mathbf{p} \mathbf{x}-\mathbf{p}^{\prime} \mathbf{x}^{\prime}\right]\right\} e^{\left\{-i t[\varepsilon(\mathbf{p})+\mu]-i t^{\prime}\left[\varepsilon\left(\mathbf{p}^{\prime}\right)+\mu\right]\right\}}}\left\langle\hat{a}_{\mathbf{p}} \hat{a}_{\mathbf{p}^{\prime}}^{\dagger}\right\rangle .
$$

For free fields the anticommutation relation

$$
\left\{\hat{a}_{\mathbf{p}}, \hat{a}_{\mathbf{p}^{\prime}}^{\dagger}\right\}=\delta_{\mathbf{p}, \mathbf{p}^{\prime}}
$$

holds, so that

$$
\begin{aligned}
\left\langle\hat{a}_{\mathbf{p}} \hat{a}_{\mathbf{p}^{\prime}}^{\dagger}\right\rangle & =\delta_{\mathbf{p}, \mathbf{p}^{\prime}}\left\{1-\left\langle\hat{a}_{\mathbf{p}^{\prime}}^{\dagger} \hat{a}_{\mathbf{p}^{\prime}}\right\rangle\right\}= \\
& =\delta_{\mathbf{p}, \mathbf{p}^{\prime}}\left\{1-f_{\mathbf{p}}\right\},
\end{aligned}
$$

where $f_{\mathbf{p}}$ is the Fermi distribution function only for the case in which we treat a gas in equilibrium, otherwise it represents a-priori unkown fermionic non-equilibrium distribution function. Inserting the last relation into Eq. (2.15) results in the expression

$$
\left\langle\psi(\mathbf{x}, t) \psi^{\dagger}\left(\mathbf{x}^{\prime}, t^{\prime}\right)\right\rangle=\frac{1}{V} \sum_{\mathbf{p}} e^{\left\{i\left[\mathbf{p}\left(\mathbf{x}-\mathbf{x}^{\prime}\right)\right]\right\}} e^{\left\{-i[\varepsilon(\mathbf{p})+\mu]\left(t-t^{\prime}\right)\right\}}\left\{1-f_{\mathbf{p}}\right\} .
$$

By taking the Fourier transform with respect to $\mathbf{x}-\mathbf{x}^{\prime}$ and $t-t^{\prime}$ of Eq. (2.16) we finally obtain

$$
G_{0}^{-+}(\omega, \mathbf{p})=-2 \pi i\left\{1-f_{\mathbf{p}}\right\} \delta\left(\omega-\varepsilon_{\mathbf{p}}+\mu\right) .
$$

We can now apply the same procedure to the other correlation functions with the result

$$
\begin{gathered}
G_{0}^{+-}(\omega, \mathbf{p})=2 \pi i f_{\mathbf{p}} \delta\left(\omega-\varepsilon_{\mathbf{p}}+\mu\right) \\
G_{0}^{--}(\omega, \mathbf{p})=[\omega-\varepsilon(\mathbf{p})+\mu+i \eta]^{-1} \pm 2 \pi i f_{\mathbf{p}} \delta\left(\omega-\varepsilon_{\mathbf{p}}+\mu\right) \\
G_{0}^{++}(\omega, \mathbf{p})=-\left[G_{0}^{--}(\omega, \mathbf{p})\right]^{*} \\
G_{0}^{R}(\omega, \mathbf{p})=[\omega-\varepsilon(\mathbf{p})+\mu+i \eta]^{-1}=\left[G_{0}^{A}(\omega, \mathbf{p})\right]^{*} .
\end{gathered}
$$

From the physical point of view Eqs. (2.17)-(2.18) contain informations about the state of the system (through the distribution function) and the excitation spectrum 
(through the delta function). The special form of Eq. (2.21) decouples the information about the excitation spectrum and the state of the system described by the non-equilibrium distribution function $f_{\mathbf{p}}[10,12]$. Before finishing this section, it is worth to explain how the physical picture changes when we return back to equilibrium. The non-equilibrium distribution function $f_{\mathbf{p}}$ then becomes the usual Fermi distribution and consequently the correlation functions become functions of the temperature and the chemical potential. This means that this formalism can also be applied to describe systems in equilibrium at $T \neq 0$.

\subsection{Perturbative Expansion}

The construction of the perturbation theory for systems in non-equilibrium follows the same steps as the equilibrium case at $T=0$ [52]. In order to illustrate how the technique works we choose, as example, Eq. (2.2) for the time-ordered Green function $G^{--}$. When we change the representation from the Heisenberg to the interaction picture, we obtain [53]

$$
G^{--}(1,2)=-i\left\langle\hat{S^{-1}} T_{C_{K}}\left[\hat{\psi}(1) \hat{\psi}^{\dagger}(2) \hat{S}\right]\right\rangle,
$$

where $\hat{\psi}, \hat{\psi}^{\dagger}$ are free fields and

$$
\begin{gathered}
\hat{S}=\hat{S}(-\infty,+\infty)=T_{K} \exp \left\{-i \int_{-\infty}^{+\infty} \hat{V}(t) d t\right\} \\
\hat{S}^{-1}=\hat{S}(+\infty,-\infty)=\tilde{T}_{K} \exp \left\{-i \int_{+\infty}^{-\infty} \hat{V}(t) d t\right\} .
\end{gathered}
$$

$\hat{V}(t)$ is the interaction operator in the interaction picture. In the following we will consider, just for simplicity reasons, a one-particle interaction

$$
\hat{V}(t)=\hat{\psi}(t)^{\dagger} \hat{U}(t) \hat{\psi}(t)
$$

The quantities $\hat{S}, \hat{S}^{-1}$ represent the scattering matrices on the two branches of the Keldysh contour and they contain both the interaction term and the external field which drives the system away from equilibrium. In other words the Keldysh path can be seen as a representation of the scattering processes induced by $\hat{S}, \hat{S}^{-1}$. In Eq. (2.22) the average is calculated with respect to a state consisting of non interacting particles and such a state can be an any state, and not only the ground state. If we were in equilibrium and at $T=0$, then we should calculate the average with respect to the ground state, therefore the action of $\hat{S}^{-1}$ could be neglected, because it would correspond just to a multiplication by a phase factor. On the other hand, any state away from equilibrium does not transform into itself under the action of $\hat{S}^{-1}$, but the resulting state can be thought as a superposition of excited states coming from 
all possible diffusion processes induced by the interaction term and by the external field. This is the reason why $\hat{S}^{-1}$ cannot be neglected any longer when we perform the perturbative expansion. As direct consequence we have a duplication of the degrees of freedom because we have now to perform a double expansion (one for both scattering matrices), whose contractions will involve and mix both sides of the contour. This gives rise to the four different Green functions we have previously introduced (Section 2.2). At first glance it seems to be an expensive price to pay, but it avoids us to handle the a priori unknown states at $t=\infty$ when propagating the system from $t=+\infty$ to $t=-\infty$, where the system's state is known [29, 41, 59]. As already anticipated, the Feynmann diagrams of the non-equilibrium perturbation theory are the result of the double expansion of the operators $\hat{S}, S^{-1}$ appearing in Eq. (2.22). Such diagrams consitute a sum of terms whose basic elements are the Wick contractions of operators pairs. Let us begin by expanding the Eq. (2.22) up to first order in the one-particle interaction. We see that the resulting expression can be divided in two parts

$$
\begin{gathered}
\left\langle T_{K} \hat{\psi}(1) \hat{\psi}^{\dagger}(2)\left[-i \int \psi(\hat{3})^{\dagger} V(3) \hat{\psi}(3) d^{4} x_{3}\right]\right\rangle+ \\
+\left\langle\left[T_{\tilde{K}} i \int \hat{\psi}^{\dagger}(3) V(3) \hat{\psi}(3) d^{4} x_{3}\right] T_{K} \hat{\psi}(1) \hat{\psi}^{\dagger}(2)\right\rangle .
\end{gathered}
$$

The first one takes into account the product of the zero-order term of $\hat{S}^{-1}$ multiplied by the first-order one of $\hat{S}$, the second term is built the other way round. In equilibrium we would have only the first term of Eq. (2.23). The two terms must then be contracted, resulting in the following products

$$
\begin{aligned}
\left\langle T_{K} \hat{\psi}(1) \hat{\psi}^{\dagger}(2)\left[-i \hat{\psi}^{\dagger}(3) U(3) \hat{\psi}(3)\right]\right\rangle & =G_{0}^{--}(3,2) G_{0}^{--}(1,3), \\
\left\langle T_{\tilde{K}}\left[i \hat{\psi}^{\dagger}(3) U \hat{\psi}(3)\right] T_{K} \hat{\psi}(1) \hat{\psi}^{\dagger}(2)\right\rangle & =G_{0}^{-+}(1,3) G_{0}^{+-}(3,2)
\end{aligned}
$$

of bare Greens functions. In Eq. (2.25) appear the lesser and greater free Green functions since we have taken two contractions involving operators belonging to two different parts of the Keldysh contour. Note that none of them is time-ordered because they mix the two sides of the contour. On the other hand, in Eq. (2.24) all operators live on the upper side of the contour, therefore we can have only time ordered functions. Equations (2.24) and (2.25) are represented graphically in Fig. 2.2. $V$ denotes the interaction potential, the arrows are the bare correlation functions $G_{0}$, and the signs $(-,+)$, stand for the upper and lower branch of the contour. Now we have all the elements to write explicitely the time ordered Green function up to the first order in $V$

$$
G_{(1)}^{(--)}(1,2)=-i^{4} \int_{-\infty}^{+\infty} d^{3} x_{3} d t_{3}\left[G_{0}^{--}(1,3) G_{0}^{--}(3,2)(-V(3))+\right.
$$






Figure 2.2: The dashed lines represent the bare interaction vertex, the continous lines the propagators connecting two points on the Keldysh contour.

$$
\left.+G_{0}^{-+}(1,3) G_{0}^{+-}(3,2) V(3)\right] .
$$

The last equation induces a dependence in $G_{(1)}^{--}$not only on $G_{0}^{--}$, but also on $G_{0}^{(-+)}$. As we will see in the following, the higher the order of the expansion and the more complicated will be such a dependence. If we proceed further and take a look at the next order $\left(O\left(V^{2}\right)\right)$, we find the graphs depicted in Fig. 2.3 whose corresponding


Figure 2.3: Second order scattering process, for the one-particle, interaction taking care of all possible sign combination of the internal indeces.

expression reads

$$
\begin{gathered}
G_{(2)}^{--}(1,2)=-i^{6} \int_{-\infty}^{+\infty} d^{3} x_{4} d^{3} x_{3} d t_{3} d t_{4} \\
{\left[G_{0}^{--}(1,4) G_{0}^{--}(4,3) G_{0}^{--}(3,2) V(3) V(4)+\right.}
\end{gathered}
$$




$$
\begin{gathered}
G_{0}^{-+}(1,4) G_{0}^{+-}(4,3) G_{0}^{--}(3,2) V(3)(-V(4))+ \\
G_{0}^{--}(1,4) G_{0}^{-+}(4,3) G_{0}^{+-}(3,2) V(3)(-V(4))+ \\
\left.G_{0}^{-+}(1,4) G_{0}^{++}(4,3) G_{0}^{+-}(3,2) V(3) V(4)\right] .
\end{gathered}
$$

The external legs are characterized by two fixed minus signs, while the two internal ones must be contracted in all possible ways. Out of all possible contractions $G_{(2)}^{--}$ acquires a dependence on all bare correlation functions, resulting in a lenghty and complicated analytical structure ${ }^{3}$. From the contour point of view this corresponds to place the internal degrees of freedom in all possible ways on both sides of the contour.

Up to now we have analyzed, just for simplicity reasons, only one-particle interaction, but the same arguments also hold for any kind of interaction. From now we will focus on two-particle interaction

$$
V(t)=\psi^{\dagger}(t) \psi^{\dagger}(t) U(t) \psi(t) \psi(t)
$$

Until now we have considered contributions up to second order, but it is possible to represent the exact $G^{--}$in a compact graphical way as shown by Fig. $2.4^{4}$.



Figure 2.4: Compact diagrammatic form of the Dyson equation for the exact $G^{--}$written in the Keldysh language.

The bold arrows stand for the exact Green functions, the thin ones denote the bare correlation functions and the circles represent the selfenergy contributions. Once again the external legs have fixed signs, while the internal ones take into account the four possible combinations of,+- signs, resulting in the selfenergies in Fig. 2.4 depicted. The full $G^{--}$can then be written as

$$
G^{--}(1,2)=G_{0}^{--}(1,2)+\int_{-\infty}^{+\infty} d^{3} x_{3} d^{3} x_{4} d t_{3} d t_{4}
$$

\footnotetext{
${ }^{3}$ The same holds also for the other three correlation functions ( Eqs. (2.4)-(2.6))

${ }^{4}$ The other exact correlation functions (Eq.(2.4)-(2.6)) have the same internal structure too, what changes are just the signs of the external legs which define the Green function we are considering.
} 


$$
\begin{gathered}
{\left[G_{0}^{--}(1,4) \Sigma^{--}(4,3) G^{--}(3,2)+\right.} \\
G_{0}^{-+}(1,4) \Sigma^{++}(4,3) G^{+-}(3,2)+ \\
G_{0}^{-+}(1,4) \Sigma^{+-}(4,3) G^{--}(3,2)+ \\
\left.G_{0}^{--}(1,4) \Sigma^{-+}(4,3) G^{+-}(3,2)\right] .
\end{gathered}
$$

The selfenergy functions or selfenergies are represented by graphs which cannot be separeted in two parts by cutting just one continous line (one particle irreducible). They can be summed as blocks depending on the order of the perturbation theory we are considering. In Fig. 2.5 we show as an example the diagrams up to the second order in $U$ for $\Sigma^{--}$and $\Sigma^{+-}$.

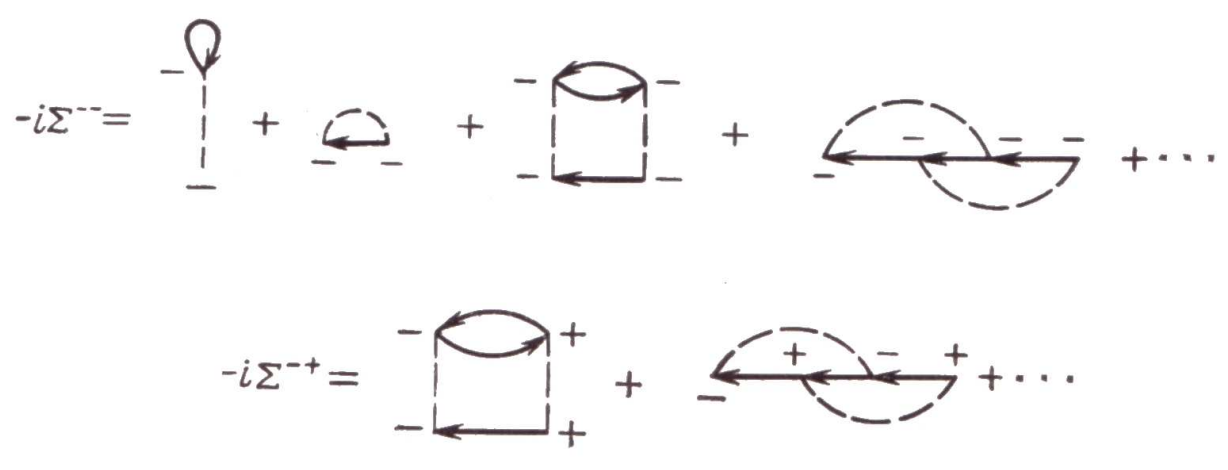

Figure 2.5: Selfenergies: $\Sigma^{--}$and $\Sigma^{-+}$up to second order.

\subsection{A useful Transformation}

In order to render the previously presented formalism easier to handle it is convenient to introduce a matrix notation, which permits to treat all correlation functions at once. To this end we define

$$
\begin{aligned}
& \hat{\Sigma}=\left(\begin{array}{cc}
\Sigma^{--} & \Sigma^{-+} \\
\Sigma^{+-} & \Sigma^{++}
\end{array}\right), \\
& \hat{G}=\left(\begin{array}{ll}
G^{--} & G^{-+} \\
G^{+-} & G^{++}
\end{array}\right) .
\end{aligned}
$$

With this matrices it is possible to rewrite the set of Dyson equations for the various Green functions in a compact matrix form as

$$
\hat{G}(1,2)=\hat{G}_{0}(1,2)+\int_{-\infty}^{+\infty} d^{3} x_{3} d^{3} x_{4} d t_{3} d t_{4}\left[\hat{G}_{0}(1,4) \hat{\Sigma}(4,3) \hat{G}(3,2)\right]
$$


We can now introduce the differential operator ${ }^{5}$

$$
\hat{G}_{0,1}^{-1}=i \frac{\partial}{\partial t_{1}}+\frac{\Delta_{1}}{2 m}+\mu
$$

which has the property $[41,53]$

$$
\hat{G_{0,1}^{-1}} \hat{G}_{0}(1,2)=\hat{\sigma}_{z} \delta(1-2)
$$

where

$$
\hat{\sigma}_{z}=\left(\begin{array}{cc}
1 & 0 \\
0 & -1
\end{array}\right)
$$

With this definition we can rewrite the Eq. (2.27) as

$$
\hat{G}_{0,1}^{-1} \hat{G}(1,2)=\hat{\sigma}_{z} \delta(1-2)+\int_{-\infty}^{+\infty} d^{3} x_{3} d t_{3}\left[\hat{\sigma}_{z} \hat{\Sigma}(4,3) \hat{G}(3,2)\right]
$$

This is a system of four integro-differential equations which are formally independent of the non-interacting state (since $G_{0}$ does not appear in Eq. (2.29)), which now enters as initial condition. In passing we emphasize that Eq. (2.29) is completely general, it for example holds in the non-stationary case and also for spatially inhomogeneous situations.

Equation (2.27) does not reflect explicitely the relation (2.29) among the Green functions. It can be made manifestly by a linear transformation

$$
\hat{R}=\frac{1}{\sqrt{2}}\left(\begin{array}{cc}
1 & 1 \\
-1 & 1
\end{array}\right)
$$

with which we obtain

$$
\begin{aligned}
& \hat{G}^{\prime}=\hat{R}^{-1} \hat{G} \hat{R}=\left(\begin{array}{cc}
0 & G^{A} \\
G^{R} & G^{K}
\end{array}\right), \\
& \hat{\Sigma^{\prime}}=\hat{R^{-1}} \hat{\Sigma} \hat{R}=\left(\begin{array}{cc}
\Sigma^{K} & \Sigma^{R} \\
\Sigma^{A} & 0
\end{array}\right),
\end{aligned}
$$

where

$$
\Sigma^{K}=\Sigma^{--}+\Sigma^{++}
$$

\footnotetext{
${ }^{5}$ If we differentiate with respect to the second variable $t_{2}$ then we must change the sign of the time derivative obtaining

$$
\begin{gathered}
\hat{G}_{0,2}^{-1}=-i \frac{\partial}{\partial t_{2}}+\frac{\Delta_{2}}{2 m}+\mu, \\
G_{0,2}^{-1} G_{0}^{--}(1,2)=\delta(1-2) .
\end{gathered}
$$
}


and

$$
G^{K}:=G^{-+}+G^{+-}=G^{--}+G^{++},
$$

For $\Sigma^{R}$ and $\Sigma^{A}$ one finally obtains, by means of $\Sigma^{--}+\Sigma^{++}=-\left(\Sigma^{+-}+\Sigma^{-+}\right)$[52], the relations

$$
\begin{aligned}
& \Sigma^{R}=\Sigma^{--}+\Sigma^{-+}, \\
& \Sigma^{A}=\Sigma^{--}+\Sigma^{+-} .
\end{aligned}
$$

We should remark that the transformation used here is not unique, in fact conventionally a different transformation is adopted, which leads to the Green function [72]

$$
\hat{G}^{\prime \prime}=\left(\begin{array}{cc}
G^{R} & G^{K} \\
0 & G^{A}
\end{array}\right)
$$

Let us write explicitely Eq. (2.27) for the transformed matrix $G^{\prime}$

$$
\begin{aligned}
& \left(\begin{array}{cc}
0 & G^{A} \\
G^{R} & G^{K}
\end{array}\right)=\left(\begin{array}{cc}
0 & G_{0}^{A} \\
G_{0}^{R} & G_{0}^{K}
\end{array}\right)+ \\
& \int_{-\infty}^{+\infty} d^{3} x_{3} d^{3} x_{4} d t_{3} d t_{4}\left[\begin{array}{cc}
0 & G_{0}^{A} \Sigma^{a} G^{a} \\
G_{0}^{R} \Sigma^{R} G^{R} & \left(G_{0}^{R} \Sigma^{K}+G_{0}^{K} \Sigma^{A}\right) G^{A}+G_{0}^{R} \Sigma^{R} G^{K}
\end{array}\right] .
\end{aligned}
$$

From the off diagonal elements we obtain the expressions for the retarded and advanced Green functions as

$$
\begin{gathered}
G^{R, A}(1,2)=G_{0}^{R, A}(1,2)+\int_{-\infty}^{+\infty} d^{3} x_{3} d^{3} x_{4} d t_{3} d t_{4} \\
{\left[G_{0}^{R, A}(1,4) \Sigma^{R, A}(4,3) G^{R, A}(3,2)\right] .}
\end{gathered}
$$

The solution to (2.31) describes the excitation spectrum of the problem we are studying. The lower right matrix element of Eq. (2.30) contains the information about the thermodynamic state of the system. Applying the differential operator $\hat{G}_{(0), 1}^{-1}$ to it, the complicated expression simplifies to

$$
\hat{G}_{(0), 1}^{-1} G^{K}(1,2)=\int_{-\infty}^{+\infty} d^{3} x_{3} d t_{3}\left[\Sigma^{K}(1,3) G^{A}(3,2)+\Sigma^{R}(1,3) G^{K}(3,2)\right],
$$

where we made use of the relation $([41])$

$$
\hat{G}_{(0), 1}^{-1} G_{(0)}^{K}=\hat{G}_{(0), 1}^{-1}\left[G_{0}^{-+}+G_{0}^{+-}\right]=0
$$

As before, the system (2.31) and (2.32) does not explicitely contain the thermodynamic state of the non-interacting system. It enters only through the initial condition to (2.32), which can be seen as a quantum mechanic generalization of the Boltzmann equation. 


\section{Functional Renormalization Group}

\subsection{Introduction}

The reliable calculation of physical properties of interacting quantum mechanical systems presents a formidable task. Typically, one has to cope with the interplay of different energy-scales possibly covering several orders of magnitude even for simple situations. Approximate tools like perturbation theory, but even numerically exact techniques can usually handle only a restricted window of energy scales and are furthermore limited in their applicability by the approximations involved or the computational resources available. In addition due to the divergence of certain classes of Feynman diagrams some of the interesting many-particle problems cannot be tackled by straight forward perturbation theory. The situation becomes even more involved if one is interested in properties off equilibrium, in particular time-dependend situations. As discussed in Chapter 2, the standard approach for such cases is based on the Keldysh formalism for the time evolution of Green functions. In order to study interacting systems, in parameter regimes that cannot be accessed by perturbation theory, novel theoretical methods have been devised recently. These approaches are based on the general concept of the renormalization group [88] by means of which one starts from high energy scales, in order to avoid infrared divergencies, and works ones way down to the desired low energy region in a systematic way, which depends on the particular problem to analyze. In particular, for interacting quantum many-particles systems in equilibrium two different schemes attempting a problem independent prescription have been developed during the nineties. One is the flow-equations technique $[20,86]$, the other is a field theoretical approach which will be explained in the course of the present chapter.

This method starts from a functional representation of the partition function of the system, serving as generating functional for Green functions. It has become known as functional renormalization group (FRG) [64, 69, 75, 87]. The aim of this chapter is to derive an extension of the FRG formalism to non-equilibrium. The general idea is to set up a generating functional, using an appropriate action on the Keldysh contour (see section 3.2), through which we obtain our correlation functions as functional derivatives. We will see that the extension to non-equilibrium does not change the formal structure of the FRG. However, there is a price to pay, viz one 
has to care for the Keldysh indices. Each of them takes into account a branch of the Keldysh contour. As direct consequence we obtain a system of differential equations, which shows a tensor structure with respect to the Keldysh indeces resulting in more complicated form compared to the equilibrium case. It is also important to point out that the formalism we are going to present is completely general and therefore can be applied to bosonic or fermionic systems in stationary state or to time-dependent problems.

Before finishing this section, it is important to mention that the Keldysh technique contains the equilibrium theory (as soon as we turn off the external field which drives the system out of equilibrium) both at $T=0$ and at finite temperatures. Besides, since the Keldysh method relies on real variables, we do not need, in contrast to the Matsubara technique, any analytical prolongation from the imaginary axis to the real one. This will permit to the non-equilibrium FRG to treat equilibrium situations too (see Sections 5.3 and 6.3.1), avoiding the analytical prolongation which may be, for some problems, a cumbersome step ${ }^{1}$.

\subsection{Extension to Non-Equilibrium}

The derivation of the non-equilibrium FRG scheme closely follows the general lines given in $[30,31]$. To this end, we will first develop a formulation that allows to express all interesting quantities via functional derivatives of a generating functional whose choice has been inspired by Kameneev's approach [41]. To set up a functional integral representation of the generating functional respecting the Keldysh time ordering, we define the matrix

$$
\hat{G}\left(\xi, \xi^{\prime}\right):=\left(\begin{array}{cc}
G^{--}\left(\xi, \xi^{\prime}\right) & G^{-+}\left(\xi, \xi^{\prime}\right) \\
G^{+-}\left(\xi, \xi^{\prime}\right) & G^{++}\left(\xi, \xi^{\prime}\right)
\end{array}\right)
$$

where the matrix elements are given by Eq. (2.3)-(2.6). The arguments $\xi, \xi^{\prime}$ are a combination of all relevant quantum numbers, position and time. For all quantities living on the Keldysh contour we introduce the short hand notation

$$
(\bar{\psi}, \mathcal{O} \psi)=i \int_{-\infty}^{+\infty} d \xi d \xi^{\prime} \bar{\psi}(\xi) \mathcal{O}\left(\xi, \xi^{\prime}\right) \psi\left(\xi^{\prime}\right)
$$

\footnotetext{
${ }^{1}$ In contrast to the Matsubara technique, where the frequency and the temperature are artificially linked by the relation (fermionic case)

$$
\omega_{n}=\frac{\pi(2 n+1)}{\beta},
$$

within the non-equilibrium FRG the dynamical degrees of freedom and the temperature are decoupled.
} 
where $\mathcal{O}$ is a matrix in the Keldysh indices and

$$
\psi\left(\xi, \xi^{\prime}\right)=\left(\begin{array}{l}
\psi_{-}\left(\xi, \xi^{\prime}\right) \\
\psi_{+}\left(\xi, \xi^{\prime}\right)
\end{array}\right)
$$

is a vector of fields (Grassmann for fermions or complex for bosons) with the time argument of $\psi_{-}$on the upper branch of the Keldysh contour and $\psi_{+}$a time argument on the lower. Later it will also prove useful to Fourier transform from time $t$ to frequency $\omega$. One then has to replace $t$ in $\xi$ by $\omega$. The integrals over $\xi$ and $\xi^{\prime}$ stand for summations over the quantum numbers and integrations over space and time or frequency. The following steps can be performed with $\xi$ either containing time or frequency. The generalization of the functional integral representation of the partition function to non-equilibrium is [41]

$$
\Xi=\frac{1}{\Xi_{0}} \int \mathcal{D} \bar{\psi} \psi \exp \left\{\left(\bar{\psi},\left[\hat{G}_{0}\right]^{-1} \psi\right)-i S_{\mathrm{int}}(\{\bar{\psi}\},\{\psi\})\right\}
$$

$\Xi_{0}$ being a normalization factor given by

$$
\Xi_{0}=\int \mathcal{D} \bar{\psi} \psi \exp \left\{\left(\bar{\psi},\left[\hat{G}_{0}\right]^{-1} \psi\right)\right\}
$$

The matrix $\hat{G}_{0}$ denotes the propagator of a suitably chosen reference system and $S_{\text {int }}$ represents an arbitrary interaction term. In order to build the generating functional for the $m$-particle Green functions we have to insert in Eq. (3.2) external source fields $\eta, \bar{\eta}$ according to (for the standard procedure in equilibrium see for example [65]

$$
\begin{aligned}
\mathcal{W}(\{\bar{\eta}\},\{\eta\})= & \frac{1}{\Xi_{0}} \int \mathcal{D} \bar{\psi} \psi \exp \left\{\left(\bar{\psi},\left[\hat{G}_{0}\right]^{-1} \psi\right)-i S_{\text {int }}(\{\bar{\psi}\},\{\psi\})\right. \\
& -(\bar{\psi}, \eta)-(\bar{\eta}, \psi)\} .
\end{aligned}
$$

Taking the logarithm of the latter equation we finally get the generator of the (connected) $m$-particle Greens functions

$$
\mathcal{W}^{c}(\{\bar{\eta}\},\{\eta\})=\ln [\mathcal{W}(\{\bar{\eta}\},\{\eta\})] .
$$

The (connected) $m$-particle Green function $G_{m}^{(c)}$ can be then obtained by taking the functional derivatives with respect to the vectors $\eta$

$$
G_{m}^{(c)}\left(\xi_{1}^{\prime}, \ldots, \xi_{m}^{\prime} ; \xi_{1}, \ldots, \xi_{m}\right)=\left.(\zeta i)^{m} \frac{\delta^{m}}{\delta \bar{\eta}_{\xi_{1}^{\prime}} \ldots \delta \bar{\eta}_{\xi_{m}^{\prime}}} \frac{\delta^{m}}{\delta \eta_{\xi_{m}} \ldots \delta \eta_{\xi_{1}}} \mathcal{W}^{(c)}(\{\bar{\eta}\},\{\eta\})\right|_{\eta=0=\bar{\eta}}
$$

At this point it's important to underline that, since the fields $\eta$ have two components (depending on the branch of the Keldysh contour), the (connected) $m$-particle Green 
functions, resulting from Eq. (3.5), must be read as tensors with respect to the Keldysh indices. Before giving a practical example of how such a tensor form arises, let us perform one further step which will bring us to the definition of the generating functional for the one-particle irreducible vertex functions $\gamma_{m}$. Introducing the fields

$$
\begin{aligned}
\phi_{\xi} & =i \frac{\delta}{\delta \bar{\eta}_{\xi}} \mathcal{W}^{c}(\{\bar{\eta}\},\{\eta\}), \\
\bar{\phi}_{\xi} & =\zeta i \frac{\delta}{\delta \eta_{\xi}} \mathcal{W}^{c}(\{\bar{\eta}\},\{\eta\}),
\end{aligned}
$$

where $\zeta= \pm 1$ and the upper sign applies to boson fields. We can perform a Legendre transformation

$$
\Gamma(\{\bar{\phi}\},\{\phi\})=-\mathcal{W}^{c}(\{\bar{\eta}\},\{\eta\})-i(\bar{\phi}, \eta)-i(\bar{\eta}, \phi)+i\left(\bar{\phi},\left[\hat{G}_{0}\right]^{-1} \phi\right) .
$$

With the help of Eq. (3.6), we define $\gamma_{m}$ as

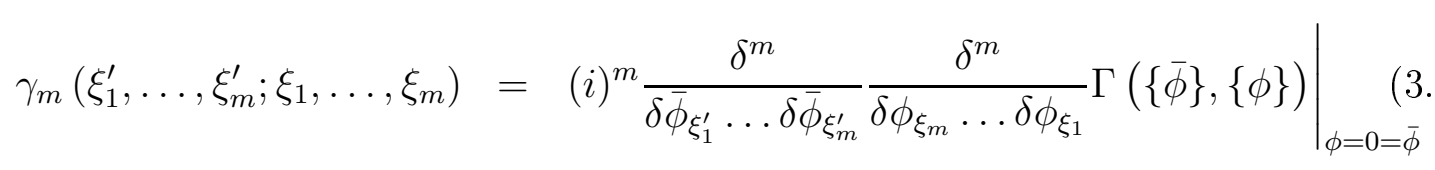

Note that in contrast to the usual definition of $\Gamma$, which consists of the first three terms in Eq. (3.6) only [65], we have added a term $\left(\bar{\phi},\left[\hat{G}_{0}\right]^{-1} \phi\right)$. The reason for introducing this term will become clear in the next section. The general relation between the $G_{m}^{(c)}$ and $\gamma_{m}$ can be found in text books [65]. For the 1-particle Green function we obtain

$$
\begin{aligned}
G_{1}\left(\xi^{\prime} ; \xi\right) & =G_{1}^{c}\left(\xi^{\prime} ; \xi\right) \\
& =i \frac{\delta}{\delta \bar{\eta}_{\xi_{1}^{\prime}}} \frac{\delta}{\delta \eta_{\xi_{1}}} \mathcal{W}^{c}=-\zeta \hat{G}_{\xi^{\prime}, \xi} \\
& =\left[\gamma_{1}-\zeta\left[\hat{G}_{0}\right]^{-1}\right]_{\xi^{\prime}, \xi}^{-1}
\end{aligned}
$$

where

$$
\hat{G}_{\xi^{\prime}, \xi}=\left[\left[\hat{G}_{0}\right]^{-1}-\hat{\Sigma}\right]_{\xi^{\prime}, \xi}^{-1},
$$

with the proper one particle selfenergy $\hat{\Sigma}$. This implies the relation $\hat{\Sigma}=\zeta \gamma_{1}$. Note that in the last equation the matrix structure appears not only with respect to $\xi$ and $\xi^{\prime}$ but also with respect to the Keldysh indices. We now intend to show how the tensor strucure of the vertices arises starting from the general expression

$$
G_{m}^{c}\left(\xi_{1}^{\prime} \ldots, \xi_{m}^{\prime} ; \xi_{1}, \ldots, \xi_{m}\right)=
$$




$$
=\left.(\zeta i)^{m} \frac{\delta^{m}}{\delta \bar{\eta}_{\xi_{1}^{\prime}} \ldots \delta \bar{\eta}_{\xi_{m}^{\prime}}} \otimes \frac{\delta^{m}}{\delta \eta_{\xi_{m}} \ldots \delta \eta_{\xi_{1}}} \mathcal{W}^{c}(\{\bar{\eta}\},\{\eta\})\right|_{\eta=0=\bar{\eta}} .
$$

We look at the easiest case $m=1$. We have two functional derivatives with respect to the vectors $\eta$, which can be made explicit by using a tensor product notation

$$
G_{1}^{c}\left(\xi^{\prime}, \xi\right)=\left.(\zeta i) \frac{\delta}{\delta \bar{\eta}_{\xi^{\prime}}} \otimes \frac{\delta}{\delta \eta_{\xi}} \mathcal{W}^{c}(\{\bar{\eta}\},\{\eta\})\right|_{\eta=0=\bar{\eta}},
$$

leading to the matrix

$$
\begin{aligned}
G_{1}^{c}\left(\xi_{1}^{\prime}, \xi_{1}\right) & =(\zeta i)\left(\begin{array}{cc}
\frac{\delta^{2} \mathcal{W}^{c}}{\left.\delta \bar{\eta}_{-}\left(\xi^{\prime}\right)\right) \delta \eta_{-}(\xi)} & \frac{\delta^{2} \mathcal{W}^{c}}{\delta \bar{\eta}_{-}\left(\xi^{\prime}\right) \delta \eta_{+}(\xi)} \\
\frac{\delta^{2} \mathcal{W}^{c}}{\delta \bar{\eta}_{+}\left(\xi^{\prime}\right) \delta \eta_{-}(\xi)} & \frac{\delta^{2} \mathcal{W}^{c}}{\delta \bar{\eta}_{+}\left(\xi^{\prime}\right) \delta \eta_{+}(\xi)}
\end{array}\right)= \\
& =\left(\begin{array}{cc}
G_{1}^{--}\left(\xi^{\prime}, \xi\right) & G_{1}^{-+}\left(\xi^{\prime}, \xi\right) \\
G_{1}^{+-}\left(\xi^{\prime}, \xi\right) & G_{1}^{++}\left(\xi^{\prime}, \xi\right)
\end{array}\right) .
\end{aligned}
$$

The extension to higher order is evident. For $m=2$ we get a four index tensor and so on for higher values of $m$. In practice we have to add, for each $m$, two indeces coming from all the possible combinations of the Keldysh components of the fields in the functional derivative. Once more we want to emphasize that our formalism has been formulated without assuming translational invariance up to now.

\subsection{The Flow Equations}

Now that we have pointed out how the Keldysh concept modifies the structure of the Green functions, we can start to derivate the FRG flow equations, following the steps in [30,31]. In Eq. (3.2) and (3.3) we replace the noninteracting propagator by a propagator $\hat{G}_{0}^{\Lambda}$ depending on a parameter $\Lambda \in\left[\Lambda_{0}, 0\right]$ and require

$$
\hat{G}_{0}^{\Lambda_{0}}=0 \quad, \quad \hat{G}_{0}^{\Lambda=0}=\hat{G}_{0},
$$

i.e. at the starting point $\Lambda=\Lambda_{0}$ no degrees of freedom are "turned on" while at $\Lambda=0$ full system is recovered. In models with infrared divergencies $\Lambda$ can be used to regularize the problem. In equilibrium this is often be achieved by implementing $\Lambda$ as an infrared cutoff in momentum or energy. One of the advantages of the FRG approach over other RG schemes is that one is not restricted to these choices and other ways of introducing the parameter $\Lambda$ have turned out to be useful for equilibrium problems [35, 61]. All that is required to derive the fundamental flow equations are the conditions Eq. (3.10). In our application of the non-equilibrium FRG to the steady state transport through an interacting quantum dot it is natural to implement $\Lambda$ as an energy cutoff. However, such a choice must not be the natural one in cases where one is interested in studying time-dependent phenomena. In 
this situation the propagator and the vertex functions in general depend on the various spatial and time variables individually and there is no obvious momentum or energy cutoff scheme. Within the FRG several ways of introducing $\Lambda$ can be worked out, compared and the one best suited for the problem under investigation can be identified.

Through $\hat{G}_{0}^{\Lambda}$ the quantities defined in Eqs. (3.2) to (3.7) acquire a $\Lambda$-dependence. Taking the derivative with respect to $\Lambda$ results in a functional differential equation for $\Gamma^{\Lambda}$. From this, by expanding in powers of the external sources, an infinite hierarchy of coupled differential equations for the $\gamma_{m}^{\Lambda}$ is obtained. Although the steps in the derivation are formally equivalent to Ref. [30,31], because of the realtime formulation additional factors $i$ and signs appear in several places. We thus believe that it is helpful to present the details of the derivation.

As a first step we differentiate $\mathcal{W}^{c, \Lambda}$ with respect to $\Lambda$, which after straightforward but lenghty algebra (see Appendix 3) leads to

$$
\frac{d}{d \Lambda} \mathcal{W}^{c, \Lambda}=\zeta \operatorname{Tr}\left(\hat{\mathcal{Q}}^{\Lambda} \hat{G}^{0, \Lambda}\right)+i \zeta \operatorname{Tr}\left(\hat{\mathcal{Q}}^{\Lambda} \frac{\delta^{2} \mathcal{W}^{c, \Lambda}}{\delta \bar{\eta} \delta \eta}\right)+\left(\frac{\delta \mathcal{W}^{c, \Lambda}}{\delta \eta}, \hat{\mathcal{Q}}^{\Lambda} \frac{\delta \mathcal{W}^{c, \Lambda}}{\delta \bar{\eta}}\right)
$$

Considering $\phi$ and $\bar{\phi}$ as the fundamental variables we obtain from Eq. (3.6)

$$
\begin{gathered}
\frac{d}{d \Lambda} \Gamma^{\Lambda}(\{\bar{\phi}\},\{\phi\})=-\frac{d}{d \Lambda} \mathcal{W}^{c, \Lambda}\left(\left\{\bar{\eta}^{\Lambda}\right\},\left\{\eta^{\Lambda}\right\}\right)- \\
-\left(\bar{\phi}, \frac{d}{d \Lambda} \eta^{\Lambda}\right)-\left(\frac{d}{d \Lambda} \bar{\eta}^{\Lambda}, \phi\right)+\left(\bar{\phi}, \hat{\mathcal{Q}}^{\Lambda} \phi\right) .
\end{gathered}
$$

Applying the chain rule and using Eq. (3.11) this leads to

$$
\frac{d}{d \Lambda} \Gamma^{\Lambda}=-\zeta \operatorname{Tr}\left(\hat{\mathcal{Q}}^{\Lambda} \hat{G}^{0, \Lambda}\right)-i \zeta \operatorname{Tr}\left(\hat{\mathcal{Q}}^{\Lambda} \frac{\delta^{2} \mathcal{W}^{c, \Lambda}}{\delta \bar{\eta}^{\Lambda} \delta \eta^{\Lambda}}\right)
$$

where the last term in Eq. (3.6) cancels a corresponding contribution arising in Eq. (3.11), thus a posterior justifying the inclusion of this term. Extending the well known relation [65] between the second functional derivatives of $\Gamma$ and $\mathcal{W}^{c}$ to non-equilibrium we obtain the functional differential equation

$$
\frac{d}{d \Lambda} \Gamma^{\Lambda}=-\zeta \operatorname{Tr}\left(\hat{\mathcal{Q}}^{\Lambda} \hat{G}^{0, \Lambda}\right)-\operatorname{Tr}\left(\hat{\mathcal{Q}}^{\Lambda} \mathcal{V}_{\bar{\phi}, \phi}^{1,1}\left(\Gamma^{\Lambda}, \hat{G}^{0, \Lambda}\right)\right)
$$

where $\mathcal{V}_{\bar{\phi}, \phi}^{1,1}$ stands for the upper left block of the matrix

$$
\mathcal{V}_{\bar{\phi}, \phi}\left(\Gamma^{\Lambda}, \hat{G}^{0, \Lambda}\right)=\left(\begin{array}{cc}
i \frac{\delta^{2} \Gamma^{\Lambda}}{\delta \phi \delta \phi}-\zeta\left[\hat{G}^{0, \Lambda}\right]^{-1} & i \frac{\delta^{2} \Gamma^{\Lambda}}{\delta \phi \delta \phi} \\
i \zeta \frac{\delta^{2} \Gamma^{\Lambda}}{\delta \phi \delta \phi} & -\left\{i \frac{\delta^{2} \Gamma^{\Lambda}}{\delta \phi \delta \phi}+\left(\left[\hat{G}^{0, \Lambda}\right]^{-1}\right)^{T}\right\}
\end{array}\right)^{-1}
$$


and the upper index $T$ denotes the transposed matrix. To obtain differential equations for the $\gamma_{m}^{\Lambda}$ which include selfenergy corrections we express $\mathcal{V}_{\bar{\phi}, \phi}$ in terms of $\hat{G}^{\Lambda}$ instead of $\hat{G}^{0, \Lambda}$. This is achieved by defining

$$
\mathcal{U}_{\bar{\phi}, \phi}=i \frac{\delta^{2} \Gamma^{\Lambda}}{\delta \bar{\phi} \delta \phi}-\gamma_{1}^{\Lambda}
$$

and using

$$
\hat{G}^{\Lambda}=\left[\left[\hat{G}^{0, \Lambda}\right]^{-1}-\zeta \gamma_{1}^{\Lambda}\right]^{-1}
$$

which leads to

$$
\frac{d}{d \Lambda} \Gamma^{\Lambda}=-\zeta \operatorname{Tr}\left(\hat{\mathcal{Q}}^{\Lambda} \hat{G}^{0, \Lambda}\right)+\zeta \operatorname{Tr}\left[\hat{G}^{\Lambda} \hat{\mathcal{Q}}^{\Lambda} \tilde{\mathcal{V}}_{\bar{\phi}, \phi}^{1,1}\left(\Gamma^{\Lambda}, \hat{G}^{\Lambda}\right)\right]
$$

with

$$
\begin{gathered}
\tilde{\mathcal{V}}_{\bar{\phi}, \phi}\left(\Gamma^{\Lambda}, \hat{G}^{\Lambda}\right)=\left[\mathbf{1}-\left(\begin{array}{cc}
\zeta \hat{G}^{\Lambda} & 0 \\
0 & {\left[\hat{G}^{\Lambda}\right]^{T}}
\end{array}\right)\left(\begin{array}{cc}
\mathcal{U}_{\bar{\phi}, \phi} & i \frac{\delta^{2} \Gamma^{\Lambda}}{\delta \bar{\phi} \delta \bar{\phi}} \\
(i \zeta) \frac{\delta^{2} \Gamma^{\Lambda}}{\delta \phi \delta \phi} & \zeta \mathcal{U}_{\bar{\phi}, \phi}^{t}
\end{array}\right)\right]^{-1} \\
\hat{\mathcal{Q}}^{\Lambda}=\frac{d}{d \Lambda}\left[\hat{G}_{0}^{\Lambda}\right]^{-1}
\end{gathered}
$$

It is important to note that $\mathcal{U}_{\bar{\phi}, \phi}$ as well as $\frac{\delta^{2} \Gamma^{\Lambda}}{\delta \phi \delta \phi}$ and $\frac{\delta^{2} \Gamma^{\Lambda}}{\delta \phi \delta \phi}$ are at least quadratic in the external sources. The initial condition for the exact functional differential equation (3.15) can either be obtained by lengthy but straightforward algebra, which we are not going to present here, or by the following simple argument: At $\Lambda=\Lambda_{0}, \hat{G}^{0, \Lambda_{0}}=0$ (no degrees of freedom are "turned on") and in a perturbative expansion of the $\gamma_{m}^{\Lambda_{0}}$ the only term which does not vanish is the bare two-particle vertex. We thus find

$$
\Gamma^{\Lambda_{0}}(\{\bar{\phi}\},\{\phi\})=S_{\text {int }}(\{\bar{\phi}\},\{\phi\}) .
$$

By expanding $\tilde{\mathcal{V}}$ in a geometric series

$$
\begin{gathered}
\tilde{\mathcal{V}}_{\bar{\phi}, \phi}=\mathbf{1}+\zeta \hat{G}^{\Lambda} \mathcal{U}_{\bar{\phi}, \phi}+\hat{G}^{\Lambda} \mathcal{U}_{\bar{\phi}, \phi} \hat{G}^{\Lambda} \mathcal{U}_{\bar{\phi}, \phi}+ \\
+\zeta^{3} i^{2} \hat{G}^{\Lambda} \frac{\delta^{2} \Gamma}{\delta \bar{\phi} \delta \bar{\phi}}\left[\hat{G}^{\Lambda}\right]^{T} \frac{\delta^{2} \Gamma}{\delta \phi \delta \phi}+\ldots
\end{gathered}
$$

and $\Gamma^{\Lambda}$ with respect to the external sources

$$
\begin{aligned}
& \Gamma^{\Lambda}(\{\bar{\phi}\},\{\phi\})=\sum_{m=0}^{\infty} \frac{(i \zeta)^{m}}{(m !)^{2}} \sum_{\xi_{1}^{\prime}, \ldots, \xi_{m}^{\prime}} \sum_{\xi_{1}, \ldots, \xi_{m}} \gamma_{m}^{\Lambda}\left(\xi_{1}^{\prime}, \ldots, \xi_{m}^{\prime} ; \xi_{1}, \ldots, \xi_{m}\right) \\
& \times \bar{\phi}_{\xi_{1}^{\prime}} \ldots \bar{\phi}_{\xi_{m}^{\prime}} \phi_{\xi_{m}} \ldots \phi_{\xi_{1}} .
\end{aligned}
$$


an exact infinite hierachy of flow equations for the $\gamma_{m}^{\Lambda}$ can be obtained. Consider as simplest example the flow equation for the single-particle vertex $\gamma_{1}$ (selfenergy). It can be derived by taking the expansion Eq. (3.19) up to first order in $\mathcal{U}$, insert it into Eq. (3.15), replace $\Gamma^{\Lambda}$ on both sides with the expansion (3.20) and compare expressions with the same powers in the fields, i.e. for $\gamma_{1}$ up to order $m=1$. This procedure leads to the expression

$$
\begin{aligned}
\frac{d}{d \Lambda} \gamma_{1}^{\Lambda}\left(\xi^{\prime} ; \xi\right) & =\zeta \frac{d}{d \Lambda} \hat{\Sigma}^{\Lambda}\left(\xi^{\prime}, \xi\right) \\
& =\operatorname{Tr}\left[\hat{\mathcal{S}}^{\Lambda} \gamma_{2}^{\Lambda}\left(\xi^{\prime}, \cdot ; \xi, \cdot\right)\right]
\end{aligned}
$$

which can be visualized by the diagram in Fig. 3.1. In order to avoid any possible

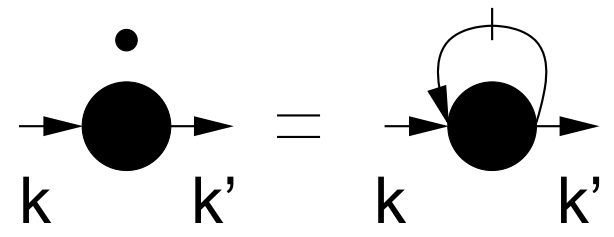

Figure 3.1: Diagrammatic form of the flow equation for $\gamma_{1}^{\Lambda}$. The slashed line stands for the single scale propagator $\hat{\mathcal{S}}^{\Lambda}$.

misunderstanding it is worth to explain that the trace in (3.20) represents more than the usual matrix trace. It contains all possible sums (integrals, series, trace, contractions) over the internal variables (quantum numbers, time, frequency, etc), or in other words, over all variables not explicitely written. In Eq. (3.20) appears the so-called single scale propagator (the slashed line in Fig. 3.1)

$$
\hat{\mathcal{S}}^{\Lambda}=\hat{G}^{\Lambda} \hat{\mathcal{Q}}^{\Lambda} \hat{G}^{\Lambda},
$$

and the quantity $\gamma_{2}^{\Lambda}\left(\xi^{\prime}, \cdot ; \xi, \cdot\right)$ denotes the matrix obtained by keeping the indices $\xi$ and $\xi^{\prime}$ fixed. We thus arrive at an expression that is formally identical to Eq. (19) in $[30,31]$. The difference appears in the matrix structure, which now also contains the index components for the branches of the Keldysh contour. To make this explicit, we write out Eq. (3.20) with respect to the Keldysh indices.

$$
\zeta \frac{d}{d \Lambda} \Sigma^{\alpha \beta, \Lambda}\left(\xi^{\prime}, \xi\right)=\operatorname{Tr} \sum_{\mu \nu} \mathcal{S}^{\mu \nu, \Lambda} \gamma_{2}^{\alpha \nu ; \beta \mu, \Lambda}\left(\xi^{\prime}, \cdot ; \xi, \cdot\right) .
$$

Apparently, the derivative of $\gamma_{1}^{\Lambda}$ is determined by $\gamma_{1}^{\Lambda}$ (implicitely through $\hat{\mathcal{S}}^{\Lambda}$ ) and the two-particle vertex $\gamma_{2}^{\Lambda}$. Thus an equation for $\gamma_{2}^{\Lambda}$ is required. Here we only show the diagrammatic form of $\gamma_{2}^{\Lambda}$ in Fig. 3.2, its derivation will be given in the next section. 

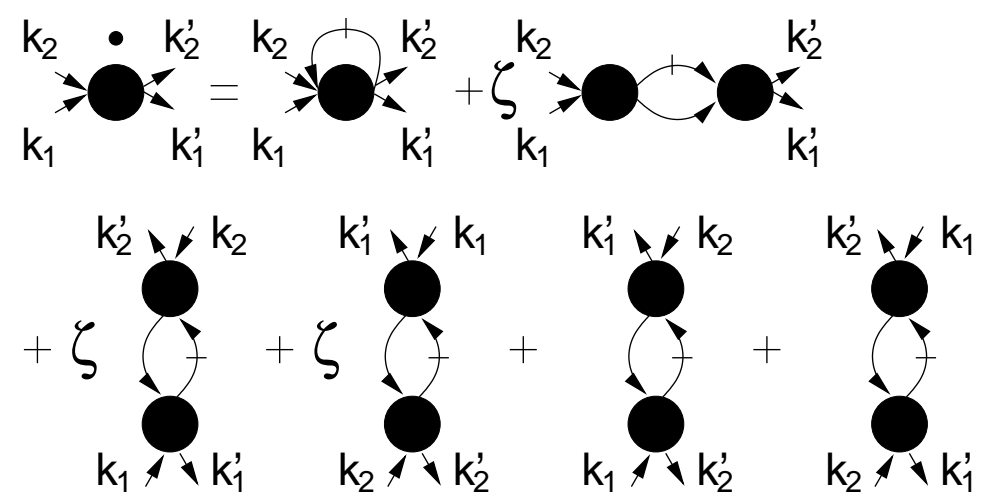

Figure 3.2: Diagrammatic form of the flow equation for $\gamma_{2}^{\Lambda}$. The slashed line stands for the single scale propagator $\hat{\mathcal{S}}^{\Lambda}$, the unslashed line for $\hat{G}^{\Lambda}$.

Since the only difference between the Eq. (3.20) and the analogous equation in $[30,31]$ are the two contour indeces, it can be interesting to have a more explicit expression of Eq. (3.20) in order to see how these two indeces change the structure of the flow equation in non-equilibrium. We have to start again from Eq. (3.20) and write explicitely the right hand side in its tensor form

$$
\begin{aligned}
& \zeta \frac{d}{d \Lambda}\left(\begin{array}{cc}
\Sigma\left(\xi^{\prime}, \xi\right)^{--, \Lambda} & \Sigma\left(\xi^{\prime}, \xi\right)^{-+, \Lambda} \\
\Sigma\left(\xi^{\prime}, \xi\right)^{+-, \Lambda} & \Sigma\left(\xi^{\prime}, \xi\right)^{++, \Lambda}
\end{array}\right)=\operatorname{Tr}\left(\mathcal{S}^{\Lambda} \gamma_{2}^{\Lambda}\left(\xi^{\prime}, \cdots ; \xi, \cdots\right)\right)=
\end{aligned}
$$



where the matrix elements $\gamma_{2}^{\Lambda}$ are tensors in the variables not explicitely written, i.e. $\left[\gamma_{2}^{\Lambda}\left(\xi^{\prime}, \cdots ; \xi, \cdots\right)\right]_{q^{\prime}, q}=\gamma_{2}^{\Lambda}\left(\xi^{\prime}, q^{\prime} ; \xi, q\right)$.

In Eq. (3.23) the product between $\hat{\mathcal{S}}^{\Lambda}$ and $\gamma_{2}^{\Lambda}\left(\xi^{\prime}, \cdots ; \xi, \cdots\right)$ must be read as a contraction among the Keldysh indeces, resulting in a sum of products among blocks having the same index pair. It is also important to point out that because of the hierarchical structure of our equations we get always a link between $2 m$-order vertex function and $2(m+1)$-order one. This means that ( case $m=1$ ) on the left hand side of Eq. (3.23) we have a two index tensor and on the right hand side a contraction between the Keldysh indeces of a two index tensor $\hat{\mathcal{S}}^{\Lambda}$ and a four index one $\gamma_{2}^{\Lambda}$. The same contraction structure remains at higher orders but it is obviously much more complicated. This point will become clear in the next section, where we study the flow equation of $\gamma_{2}$. 


\subsection{Flow Equation for the Two-Particles Vertex}

To obtain the flow equation for $\hat{\dot{\Sigma}}$ we have expanded Eq. (3.16) in a geometric series up to first order and $\Gamma^{\Lambda}$ Eq. (3.20) in the external sources up to the order $m=2$. In order to find the flow equation for the vertex function, $\dot{\gamma}_{2}$, we just have to proceed one step further, namely expand Eq. (3.16) up to the second order and $\Gamma^{\Lambda}$ up to third $(m=3)$. After comparison of the terms with the same power in the fields we obtain a differential equation for $\gamma_{2}^{\Lambda}$, which does not only contain $\gamma_{1}^{\Lambda}$ - implicitly via the propagators - and $\gamma_{2}^{\Lambda}$, but also the threeparticle vertex $\gamma_{3}^{\Lambda}$. This three-particle vertex depends on the four-particle vertex etc. It is generically impossible to solve the full set of infinitely many coupled differential equations. In applications one has to truncate it, and this is usually done at $m=2$, i.e. one replaces all vertices with $m>2$ by their initial values, which for typical problems encountered means $\gamma_{m}=0$ for $m>2$. The truncated equation for $\gamma_{2}^{\Lambda}$ then reads (see Eq. (15) by Karrasch at al. [42] and Fig. 3.2):

$$
\begin{aligned}
& \frac{d}{d \Lambda} \gamma^{\alpha \beta \gamma \delta, \Lambda}\left(\xi_{1}^{\prime}, \xi_{2}^{\prime} ; \xi_{1}, \xi_{2}\right)=\sum_{\mu, \nu, \rho, \eta} \int d \xi_{3} d \xi_{3}^{\prime} d \xi_{4} d \xi_{4}^{\prime}( \\
& G^{\rho \eta, \Lambda}\left(\xi_{3}^{\prime}, \xi_{3}\right) \mathcal{S}^{\nu \mu, \Lambda}\left(\xi_{4}, \xi_{4}^{\prime}\right)\left[\gamma^{\alpha \beta \rho \nu, \Lambda}\left(\xi_{1}^{\prime}, \xi_{2}^{\prime} ; \xi_{3}, \xi_{4}\right) \gamma^{\eta \mu \gamma \delta, \Lambda}\left(\xi_{3}^{\prime}, \xi_{4}^{\prime} ; \xi_{1}, \xi_{2}\right)\right] \\
- & G^{\eta \rho, \Lambda}\left(\xi_{3}, \xi_{3}^{\prime}\right) \mathcal{S}^{\nu \mu, \Lambda}\left(\xi_{4}, \xi_{4}^{\prime}\right)\left[\gamma^{\alpha \mu \gamma \eta, \Lambda}\left(\xi_{1}^{\prime}, \xi_{4}^{\prime} ; \xi_{1}, \xi_{3}\right) \gamma^{\rho \beta \nu \delta, \Lambda}\left(\xi_{3}^{\prime}, \xi_{2}^{\prime} ; \xi_{4}, \xi_{2}\right)\right] \\
+ & \gamma^{\alpha \rho \gamma \nu, \Lambda}\left(\xi_{1}^{\prime}, \xi_{3}^{\prime} ; \xi_{1}, \xi_{4}\right) \gamma^{\mu \beta \eta \delta, \Lambda}\left(\xi_{4}^{\prime}, \xi_{2}^{\prime} ; \xi_{3}, \xi_{2}\right) \\
- & \gamma^{\beta \mu \gamma \eta, \Lambda}\left(\xi_{2}^{\prime}, \xi_{4}^{\prime} ; \xi_{1}, \xi_{3}\right) \gamma^{\rho \alpha \nu \delta, \Lambda}\left(\xi_{3}^{\prime}, \xi_{1}^{\prime} ; \xi_{4}, \xi_{2}\right) \\
- & \left.\left.\gamma^{\beta \rho \gamma \nu, \Lambda}\left(\xi_{2}^{\prime}, \xi_{3}^{\prime} ; \xi_{1}, \xi_{4}\right) \gamma^{\mu \alpha \eta \delta, \Lambda}\left(\xi_{4}^{\prime}, \xi_{1}^{\prime} ; \xi_{3}, \xi_{2}\right)\right]\right) .
\end{aligned}
$$

Equation (3.24) has a complicated form consisting of sums and integrals over times or frequencies and quantum numbers including spins and contractions over the Keldysh indices. The main difference to equilibrium is the presence of mixed contractions regarding the Keldysh indices of the matrices $\hat{G}$ and $\hat{\mathcal{S}}$ and the fourindex tensors $\gamma_{2}$. At this point it is worth to give some more insight concerning the relation between the dynamical indeces and the Keldysh ones appearing in the Greens functions and in the tensor $\gamma_{2}$.

From the topological structure of the flow-equations (see Fig. 3.2) we see that each Keldysh index is strictly connected to a dynamical one. For instance, in $G^{\nu \mu}\left(\xi_{4}, \xi_{4}^{\prime}\right)$ the index $\nu$ is linked to $\xi_{4}$ and $\mu$ to $\xi_{4}^{\prime}$. This means that if we permute $\overbrace{\nu \mu} \rightarrow \overbrace{\mu \nu}$, then we have to rotate the corresponding dynamical indeces $\overbrace{\xi_{4}, \xi_{4}^{\prime}}^{\rightarrow} \rightarrow \overbrace{\xi_{4}^{\prime}, \xi_{4}}^{\text {too. It }}$ is also important to note that the permutation of each dynamical index induces a factor $\zeta$ in the vertex, i.e. a minus sign for fermions, which is not true for the permutation of two Keldysh indices, because they do not represent any physical variable such as impulse, spin etc. In particular we have to distinguish two cases 
when we permutate the first or the second pair of Keldysh indeces in $\gamma_{2}^{\Lambda}$. In the first case we have to transpose each block matrix (see Eq. (3.23)), in the second case the block matrices remain unchanged but we must transpose the entire tensor. 


\section{Stationary Transport through a Quantum Dot}

\subsection{Introduction}

In the previous chapter we developed the formal extension of the FRG in nonequilibrium in a form which was as general as possible. We obtained a hierarchy of differential equations, which we had to truncate at a certain level to obtain a manageable set of equations. Note that even within this truncated system the remaining set of differential equations must typically still be further approximated to allow a numerical solution $[2,36]$.

The goal of the present chapter is to apply our formalism to the single impurity Anderson model SIAM describing the transport properties of a single-level quantum dot under a constant bias at $T=0$. As discussed in the introduction, the SIAM is the simplest model displaying non trivial many-body effects. Furthermore, the FRG equations are simple enough to in principle allow for a full treatment of the truncated system including all time or frequency dependence. However, as first step, we will concentrate on the stationary case and will introduce further approximations to reduce the complexity of the FRG equations.

Let us start by deriving the analytical expressions of the non-interacting dot Green functions of the SIAM, as next step we will approximate the vertex by a energyindipendent one and discuss how the mathematical form of the non-equilibrium flow equations will change. The limit $V_{B} \rightarrow 0$, will serve as test case where we will show that our non-equilibrium FRG is able to reproduce the results in equilibrium [2]. We will show in particular that, even with such a drastic approximation, we are able to reproduce, at least qualitatively, the results regarding the current $J$ and the differential conductance $G$, which have been obtained by several authors with perturbation theory $[16,33]$.

\subsection{The Non-Interacting Case}

The SIAM has already been introduced in detail in the first chapter (see Section 1.4.2). A particularly simple case, where we can calculate all Keldysh functions exactly, is the non-interacting limit $U=0$. Besides, serving as an exercise to obtain a feeling for the structure of these correlation functions, the results of the following 


\section{$\hat{\Sigma}_{(0)}=\longleftrightarrow$}

Figure 4.1: Graphical representation of $\hat{\Sigma}_{(0)}$

calculations are also necessary to initialize the FRG equations set up in the next section. The starting point to derive the correlation functions is the Dyson equation written in a matrix form

$$
\hat{G}_{d,(0)}(\omega)=\left[\left(\hat{g}_{d,(0)}\right)^{-1}-\hat{\Sigma}_{(0)}\right]^{-1},
$$

where $\hat{G}_{d,(0)}, \hat{g}_{d,(0)}, \hat{\Sigma}_{(0)}$ are the matrices

$$
\begin{gathered}
\hat{G}_{d,(0)}=\left(\begin{array}{cc}
G_{d,(0)}^{--} & G_{d,(0)}^{-+} \\
G_{d,(0)}^{+-} & G_{d,(0)}^{++}
\end{array}\right), \\
\hat{g}_{d,(0)}=\left(\begin{array}{cc}
g_{d,(0)}^{--} & g_{d,(0)}^{-+} \\
g_{d,(0)}^{+-} & g_{d,(0)}^{++}
\end{array}\right), \\
\hat{\Sigma}_{(0)}=\frac{V^{2}}{2 N} \sum_{\vec{k} \alpha}\left(\begin{array}{cc}
G_{\vec{k} \alpha(0)}^{--} & G_{\vec{k} \alpha(0)}^{-+} \\
G_{\vec{k} \alpha(0)}^{+-} & G_{\vec{k} \alpha(0)}^{++}
\end{array}\right),
\end{gathered}
$$

(see Fig. 4.1 ) where we assume that $\varepsilon_{k, L}=\varepsilon_{k, R}$ and $V_{L}=V_{R}=V / \sqrt{2}$. The meaning of the factor $\sqrt{2}$ will become clear later. We need the explicit expressions of the free Green functions for the dot and the electrodes. They read [52]

$$
\begin{aligned}
G_{\vec{k} \alpha(0)}^{--}(\omega)= & \frac{1}{\omega-\varepsilon_{\vec{k}}+\mu_{\alpha}+i \delta} \\
& +2 i \pi f\left(\varepsilon_{\vec{k}}\right) \delta\left(\omega-\varepsilon_{\vec{k}}+\mu_{\alpha}\right), \\
G_{\vec{k} \alpha(0)}^{++}(\omega)= & -\left[G_{\vec{k} \alpha}^{--}(\omega)\right]^{*}, \\
G_{\vec{k} \alpha(0)}^{-+}(\omega)= & 2 i \pi f\left(\varepsilon_{\vec{k}}\right) \delta\left(\omega-\varepsilon_{\vec{k}}+\mu_{\alpha}\right), \\
G_{\vec{k} \alpha(0)}^{+-}(\omega)= & -2 i \pi f\left(-\varepsilon_{\vec{k}}\right) \delta\left(\omega-\varepsilon_{\vec{k}}+\mu_{\alpha}\right)
\end{aligned}
$$


for the leads and

$$
\begin{aligned}
g_{d(0)}^{--}(\omega)= & \frac{1}{\omega-\varepsilon+\mu+i \delta} \\
& +2 i \pi f(\varepsilon) \delta(\omega-\varepsilon+\mu), \\
g_{d(0)}^{++}(\omega)= & -\left[g_{d(0)}^{--}(\omega)\right]^{*}, \\
g_{d(0)}^{-+}(\omega)= & 2 i \pi f(\varepsilon) \delta(\omega-\varepsilon+\mu), \\
g_{d(0)}^{+-}(\omega)= & -2 i \pi f(-\varepsilon) \delta(\omega-\varepsilon+\mu)
\end{aligned}
$$

for the dot. Inserting Eq. (4.5)-(4.8) into Eq. (4.4) and then into Eq. (4.1) together with Eq. (4.9)-(4.12), we obtain the dot free Green function in a matrix form

$$
\begin{gathered}
\hat{G}_{d, 0}(\omega)=\frac{1}{\left(\omega-V_{G}\right)^{2}+\Gamma^{2}} \\
\left(\begin{array}{cc}
\omega-V_{G}-i \Gamma[1-F(\omega)] & i \Gamma F(\omega) \\
-i \Gamma F(-\omega) & -\left(\omega-V_{G}\right)-i \Gamma[1-F(\omega)]
\end{array}\right),
\end{gathered}
$$

where $F( \pm \omega):=f_{L}( \pm \omega)+f_{R}( \pm \omega)$ and $f_{\alpha}( \pm \omega):=f\left( \pm\left(\omega-\mu_{\alpha}\right)\right)$ are the Fermi functions of the reservoirs and $\Gamma=\pi V^{2} N_{F}$, (with $N_{F}$ the density of states at the Fermi level in the reservoirs) is the tunnel rate between the leads and the dot.

We now explain the procedure to derive the matrix elements in Eq. (4.13). Let us, for instance, start by calculating the selfenergy component

$$
\Sigma_{(0)}^{--}=\frac{V^{2}}{2 N} \sum_{\vec{k} \alpha} G_{\vec{k} \alpha(0)}^{--} .
$$

First of all we transform the sum over $\vec{k}$ into an integral

$$
\frac{1}{N} \sum_{\vec{\kappa}} \rightarrow \int_{-\infty}^{\infty} d \omega N(\omega)
$$

over the density of states $N(\omega)$. At this point we introduce a second approximation (flat band) for the density of states in which $N(\omega)$ is

$$
N(\omega)= \begin{cases}N_{F}, & \text { if }|\omega|<D \\ 0, & \text { else }\end{cases}
$$

Therefore we may write Eq. (4.14) as

$$
\Sigma_{(0)}^{--}=\frac{V^{2}}{2} \sum_{\alpha} N_{F} \int_{-D}^{D} d \omega G_{\vec{k} \alpha(0)}^{--}(\omega) .
$$


Substitute Eq. (4.5) into the integral (4.16). The Dirac delta, in the second term of Eq. (4.5) simplifies the calculation of (4.16), leading to the definition

$$
\sum_{\alpha} \int_{-D}^{D} d \omega f\left(\varepsilon_{\vec{k} \alpha}\right) \delta\left(\omega-\varepsilon_{\vec{k}}+\mu_{\alpha}\right)=f_{L}\left(\varepsilon_{\vec{k}}\right)+f_{R}\left(\varepsilon_{\vec{k}}\right):=F(\omega) .
$$

The integral with respect to the first term of Eq. (4.5) can be easily calculated in the limit $D \gg \varepsilon$ (wideband limit)

$$
\int_{-D}^{D} d \omega \frac{1}{\omega+\mu_{\alpha}-\varepsilon}=\ln \left[\frac{D+\varepsilon}{D-\varepsilon}\right] \rightarrow 0
$$

leading to the result

$$
\Sigma_{(0)}^{--}=\frac{V^{2}}{2} \sum_{\alpha} N_{F} \int_{-D}^{D} d \omega G_{\vec{k} \alpha(0)}^{--}(\omega)=i \Gamma \sum_{\alpha} f_{\alpha}\left(\varepsilon_{\vec{k}}\right):=i \Gamma F(\omega) .
$$

The same procedure has to be repeated in order to calculate the other components of $\hat{\Sigma}_{(0)}$. By inserting the matrix elements of $\hat{\Sigma}_{(0)}$, together with equations (4.9)-(4.12) into Eq. (4.1), we obtain by a straightforward calculation the following matrix elements, which correspond to the free Green functions of the dot

$$
\begin{aligned}
G_{d, 0}^{--}(\omega) & =\frac{\omega-V_{G}-i \Gamma[1-F(\omega)]}{\left(\omega-V_{G}\right)^{2}+\Gamma^{2}} \\
G_{d, 0}^{++}(\omega) & =-\left[G_{d, 0}^{--}(\omega)\right]^{*} \\
G_{d, 0}^{-+}(\omega) & =i \frac{\Gamma F(\omega)}{\left(\omega-V_{G}\right)^{2}+\Gamma^{2}} \\
G_{d, 0}^{+-}(\omega) & =-i \frac{\Gamma F(-\omega)}{\left(\omega-V_{G}\right)^{2}+\Gamma^{2}}
\end{aligned}
$$

\subsection{Flow equations}

We start from the equation (3.23)

$$
\zeta \frac{d}{d \Lambda} \Sigma^{\alpha \beta, \Lambda}\left(\xi^{\prime}, \xi\right)=\operatorname{Tr} \sum_{\mu \nu} \mathcal{S}^{\mu \nu, \Lambda} \gamma_{2}^{\alpha \nu ; \beta \mu, \Lambda}\left(\xi^{\prime}, \cdot ; \xi, \cdot\right)
$$

for the selfenergy and neglect the energy dependence in the vertex

$$
\gamma_{2}^{\alpha \nu ; \beta \mu, \Lambda}\left(\xi^{\prime}, \xi_{1} ; \xi, \xi_{2}\right) \rightarrow \gamma_{2, \xi^{\prime}, \xi_{1}, \xi, \xi_{2}}^{\alpha \nu ; \beta \mu, \Lambda}
$$


The single particle quantum numbers $\xi$ in the right hand side of Eq. (4.23) represent the spin index only. As direct consequence $\hat{\Sigma}$ does not depend on energy any longer. The resulting flow equation for the selfenergy becomes

$$
\zeta \frac{d}{d \Lambda} \sum_{\xi^{\prime}, \xi}^{\alpha \beta, \Lambda}=\operatorname{Tr} \sum_{\mu, \nu} \mathcal{S}^{\mu \nu, \Lambda} \gamma_{2, \xi^{\prime}, ; \xi ;, \cdot}^{\alpha \nu ; \beta \mu, \Lambda} .
$$

Using the antisymmetry of $\gamma_{2}^{\Lambda}$ in the spin indices and the spin conservation imposed by the structure of the interaction we can further simplify Eq. (4.24) by introducing the flowing interaction $U^{\alpha \beta \gamma \delta, \Lambda}$ defined as

$$
\gamma_{\sigma_{1}^{\prime}, \sigma_{2}^{\prime} ; \sigma_{1}, \sigma_{2}}^{\alpha \beta ; \delta, \Lambda}=\delta_{\sigma_{1}^{\prime}, \sigma_{1}} \delta_{\sigma_{2}^{\prime}, \sigma_{2}} U^{\alpha \beta ; \gamma \delta, \Lambda}-\delta_{\sigma_{2}^{\prime}, \sigma_{1}} \delta_{\sigma_{1}^{\prime}, \sigma_{2}} U^{\beta \alpha ; \gamma \delta, \Lambda} .
$$

As last step we specify how the parameter $\Lambda$ is introduced. Since we are interested in a stationary situation, i.e. the propagators only depend on the time difference $t-t^{\prime}$, all equations can be transformed into frequency space and one natural choice is a frequency cutoff of the form

$$
\hat{G}_{d, 0}^{\Lambda}(\omega)=\Theta(|\omega|-\Lambda) \hat{G}_{d, 0}(\omega)
$$

with $\Lambda_{0} \rightarrow \infty$ [30]. Evaluating $\hat{\mathcal{S}}$ by means of the Morris lemma [30,64] results in (see Appendix 1 and 2)

$$
\hat{\mathcal{S}}^{\Lambda}(\omega) \rightarrow \delta(|\omega|-\Lambda) \hat{\mathcal{G}}^{\Lambda}(\omega)
$$

with

$$
\hat{\mathcal{G}}_{d}^{\Lambda}(\omega)=\frac{1}{\left[\hat{G}_{d, 0}(\omega)\right]^{-1}-\hat{\Sigma}^{\Lambda}},
$$

where we used that $\hat{\Sigma}^{\Lambda}$ does not depend on energy. Inserting Eq. (4.25)-(4.27) into Eq. (4.24), we arrive at the following expression

$$
\frac{d}{d \Lambda} \Sigma^{\alpha \beta, \Lambda}=-\frac{1}{2 \pi} \sum_{\gamma, \delta} \sum_{\omega= \pm \Lambda} \mathcal{G}_{d}^{\gamma \delta, \Lambda}(\omega)\left(2 U^{\alpha \delta \beta \gamma, \Lambda}-U^{\delta \alpha \beta \gamma, \Lambda}\right)
$$

for the flow equation of the selfenergy. Finally, the initial condition for the self-energy is $\lim _{\Lambda_{0} \rightarrow \infty} \hat{\Sigma}^{\Lambda_{0}}=0$, which means that we begin with an interaction-free problem.

Concerning the flow equation for $\gamma_{2}$ the starting point is Eq. (3.24) in which we substitute Eq. (4.23). The result can be further simplified by taking advantage of spin and energy conservation, which implies that $\hat{\mathcal{G}}$ and $\hat{\mathcal{S}}$ are diagonal with respect to $\xi_{3}=\xi_{3}^{\prime}$ and $\xi_{4}=\xi_{4}^{\prime}$. As far as Eq. (4.25) is concerned, spin conservation can be written as $\sigma_{1}=\sigma_{1}^{\prime}, \sigma_{2}=\sigma_{2}^{\prime}$ and $\sigma_{1}=\bar{\sigma}_{2}$. Note that such relations do not hold for the corresponding Keldysh indices (see Section 3.4). The integrals over $\omega_{3^{\prime}}$ and $\omega_{4^{\prime}}$ as well as the sums over $\sigma_{3}^{\prime}$ and $\sigma_{4}^{\prime}$ can then be performed straightforwardly. Using 
Morris lemma [64], we can rewrite the matrix product of $\hat{G}$ and $\hat{\mathcal{S}}$ as (see Appendix 1 and 2)

$$
\hat{G}^{\Lambda}(\omega) \hat{\mathcal{S}}^{\Lambda}\left(\omega^{\prime}\right) \rightarrow \frac{1}{2} \delta\left(\left|\omega^{\prime}\right|-\Lambda\right) \hat{\mathcal{G}}_{d}^{\Lambda}(\omega) \hat{\mathcal{G}}_{d}^{\Lambda}\left(\omega^{\prime}\right)
$$

where $\hat{\mathcal{G}}$ is defined in Eq. (4.28). The $\delta$-function can be used to perform another of the frequency integrals, and the remaining one can be evaluated because of energy conservation of the two-particle vertex. Using the spin independence of the Green function for zero magnetic field, this leads to

$$
\begin{gathered}
\frac{d}{d \Lambda} \gamma_{\sigma_{1}^{\prime}, \sigma_{2}^{\prime} ; \sigma_{1}, \sigma_{2}}^{\alpha \beta \gamma \delta, \Lambda}=\frac{1}{4 \pi} \sum_{\omega= \pm \Lambda} \sum_{\sigma_{3}, \sigma_{4}} \sum_{\mu, \nu, \rho, \eta}\left(\mathcal{G}_{d}^{\rho \eta, \Lambda}(\omega) \mathcal{G}_{d}^{\nu \mu, \Lambda}(\omega) \gamma_{\sigma_{1}^{\prime}, \sigma_{2}^{\prime} ; \sigma_{3}, \sigma_{4}}^{\alpha \rho \nu, \Lambda} \gamma_{\sigma_{3}, \sigma_{4} ; \sigma_{1}, \sigma_{2}}^{\eta \mu \gamma \delta, \Lambda}\right. \\
-\mathcal{G}_{d}^{\eta \rho, \Lambda}(\omega) \mathcal{G}_{d}^{\nu \mu, \Lambda}(\omega)\left[\gamma_{\sigma_{1}^{\prime}, \sigma_{4} ; \sigma_{1}, \sigma_{3}}^{\alpha \mu \gamma \eta,} \gamma_{\sigma_{3}, \sigma_{2}^{\prime} ; \sigma_{4}, \sigma_{2}}^{\rho \beta \nu \delta, \Lambda}+\gamma_{\sigma_{1}^{\prime}, \sigma_{3} ; \sigma_{1}, \sigma_{4}}^{\alpha \rho \gamma \nu, \Lambda} \gamma_{\sigma_{4}, \sigma_{2}^{\prime} ; \sigma_{3}, \sigma_{2}}^{\mu \beta \eta \delta, \Lambda}\right. \\
\left.\left.-\gamma_{\sigma_{2}^{\prime}, \sigma_{4} ; \sigma_{1}, \sigma_{3}}^{\beta \mu \gamma \eta, \Lambda} \gamma_{\sigma_{3}, \sigma_{1}^{\prime} ; \sigma_{4}, \sigma_{2}}^{\rho \alpha \nu \delta, \Lambda}-\gamma_{\sigma_{2}^{\prime}, \sigma_{3} ; \sigma_{1}, \sigma_{4}}^{\beta \rho \gamma \nu, \Lambda} \gamma_{\sigma_{4}, \sigma_{1}^{\prime} ; \sigma_{3}, \sigma_{2}}^{\mu \alpha \eta \delta, \Lambda}\right]\right)
\end{gathered}
$$

Comparing Eq. (4.30) to Eq. (20) in Karrasch et al. [42], where similar approximations were made in equilibrium, we see that we have two more terms because of the Keldysh indices. In the first term appears $\tilde{G}_{d}^{\rho \eta, \Lambda}$, while its transpose $\tilde{G}_{d}^{\eta \rho, \Lambda}$ enters everywhere else. As initial condition we have to set

$$
\gamma_{2}^{\alpha \alpha \alpha \alpha, \Lambda_{0}}\left(\xi_{1}^{\prime}, \xi_{2}^{\prime} ; \xi_{1}, \xi_{2}\right)=\alpha i U\left(\delta_{\sigma_{1}, \sigma_{1}^{\prime}} \delta_{\sigma_{2}, \sigma_{2}^{\prime}}-\delta_{\sigma_{1}, \sigma_{2}^{\prime}} \delta_{\sigma_{2}, \sigma_{1}^{\prime}}\right)
$$

Using the antisymmetry of $\gamma_{2}^{\Lambda}$ in the spin indices and spin conservation (Eq. (4.25)) we can further simplify Eq. (4.30) finally leading to the flow equation

$$
\begin{gathered}
\frac{d}{d \Lambda} U^{\alpha \beta \gamma \delta, \Lambda}=\frac{1}{4 \pi} \sum_{\omega= \pm \Lambda} \sum_{\mu, \nu \rho, \eta}\left(\mathcal{G}_{d}^{\rho \eta, \Lambda}(-\omega) \mathcal{G}_{d}^{\nu \mu, \Lambda}(\omega)\left[U^{\alpha \beta \rho \nu, \Lambda} U^{\eta \mu \gamma \delta, \Lambda}+U^{\beta \alpha \rho \nu, \Lambda} U^{\mu \eta \gamma \delta, \Lambda}\right]\right. \\
-\mathcal{G}_{d}^{\eta \rho, \Lambda}(\omega) \mathcal{G}_{d}^{\nu \mu, \Lambda}(\omega)\left[2 U^{\alpha \mu \gamma \eta, \Lambda} U^{\rho \beta \nu \delta, \Lambda}-U^{\alpha \mu \gamma \eta, \Lambda} U^{\beta \rho \nu \delta, \Lambda}-U^{\mu \alpha \gamma \eta, \Lambda} U^{\rho \beta \nu \delta, \Lambda}\right. \\
+2 U^{\alpha \rho \gamma \nu, \Lambda} U^{\mu \beta \eta \delta, \Lambda}-U^{\alpha \rho \gamma \nu, \Lambda} U^{\beta \mu \eta \delta, \Lambda}-U^{\rho \alpha \gamma \nu, \Lambda} U^{\mu \beta \eta \delta, \Lambda}-U^{\mu \beta \gamma \eta, \Lambda} U^{\alpha \rho \nu \delta, \Lambda} \\
\left.\left.-U^{\rho \beta \gamma \nu, \Lambda} U^{\alpha \mu \eta \delta, \Lambda}\right]\right) .
\end{gathered}
$$

From the initial value of $\gamma_{2}^{\Lambda}$ at $\Lambda=\Lambda_{0} \rightarrow \infty$ given in Eq. (4.31) we can read off as the initial value for $U^{\alpha \beta \gamma \delta, \Lambda_{0}}$

$$
U^{\alpha \alpha \alpha \alpha, \Lambda_{0}}=\alpha i U,
$$

while all other components are zero. In other words, we take as initial conditions the bare interaction.

The system of differential equations for $\hat{\Sigma}^{\Lambda}$ and $U^{\Lambda}$ Eq. (4.29)-(4.32) will be analyzed later this chapter. The selfenergy obtained from its numerical integration, must be inserted into the expressions for the current and the conductance we are going to discuss in the next section. 


\subsection{Current}

For the model (1.2) the current is given by the Meir-Wingreen formula [63, 89]

$$
J=\frac{1}{2}\left(J_{L}+J_{R}\right)=\frac{i e \Gamma}{2 \pi \hbar} \int_{-\infty}^{+\infty} d \omega\left[f_{L}(\omega)-f_{R}(\omega)\right]\left[G_{d}^{+-}(\omega)-G_{d}^{-+}(\omega)\right]
$$

with $f_{L, R}( \pm \omega):=\left[1+\exp \left\{ \pm \beta\left(\omega-\mu_{\alpha}\right)\right\}\right]$. The full interacting one-particle Green functions of the dot are denoted by $G_{d}^{+-}(\omega), G_{d}^{-+}(\omega)$ and $J_{L / R}$ are the currents across the left and right dot-lead contacts

$$
J_{L(R)}= \pm \frac{i e \Gamma}{\pi \hbar} \int_{-\infty}^{\infty} d \omega\left[f_{L(R)}(-\omega) \tilde{G}_{d}^{-+}(\omega)+f_{L(R)}(\omega) \tilde{G}_{d}^{+-}(\omega)\right]
$$

respectively. Substitute into Eq. (4.33) the expressions of the full Greens functions $G_{d}^{+-}(\omega), G_{d}^{-+}(\omega)$ leading to the following expression for $J$

$$
J=\frac{i e \Gamma}{2 \pi \hbar} \int_{-\infty}^{+\infty} d \omega\left[f_{L}(\omega)-f_{R}(\omega)\right] \frac{2 i\left[\Gamma-\Im m \Sigma^{\alpha, \beta}\right]}{\tilde{\Delta}}
$$

with

$$
\begin{gathered}
\tilde{\Delta}=\left|\omega-V_{G}+i \Gamma[1-F(\omega)]-\Sigma^{--}(\omega)\right|^{2}+\left[\Gamma F(\omega)-\Im m \Sigma^{-+}(\omega)\right], \\
F(\omega)=f_{L}(\omega)+f_{R}(\omega) .
\end{gathered}
$$

Equation (4.34) plays a central role in the course of this work, since it is the formula we will adopt to calculate the transport parameters.

Before going further it is necessary to explain in more detail how the energy independent selfenergies affect the original form of the Meir-Wingreen Formula. Let us come back now to Eq. (4.33). It has been written in a somewhat unusual form, not employing the original relation $[63,89]$

$$
G_{d}^{R}(\omega)-G_{d}^{A}(\omega)=-2 \pi i \rho_{d}(\omega)
$$

where $\rho_{d}(\omega)=\frac{-\Im m\left[G^{R}(\omega)\right]}{\pi}$ denotes the dot's one-particle spectral function. The reason is that, as soon as we neglect the energy dependence of the selfenergies $\Sigma^{\alpha \beta}$,

$$
G^{R,(A)}(\omega)=G^{--}(\omega)-G^{-+,(+-)}(\omega)
$$


does not hold any longer. This can be seen by inserting $G^{\alpha \beta}(\omega)$ in the previous equation to obtain

$$
\begin{gathered}
G_{d}^{R}(\omega)=-\frac{\omega-V_{g}-i\left[\Gamma+\Im m \Sigma^{--}-\Im m \Sigma^{-+}\right]}{\tilde{\Delta}(\omega)}, \\
G_{d}^{A}(\omega)=-\frac{\omega-V_{g}-i\left[\Gamma+\Im m \Sigma^{--}+\Im m \Sigma^{-+}-2 \Gamma(F(\omega)+F(-\omega))\right]}{\tilde{\Delta}(\omega)} .
\end{gathered}
$$

A simple comparison between the imaginary parts of these two relations directly shows that $G^{R}(\omega) \neq\left[G^{A}(\omega)\right]^{*}$, unless at least $\Sigma^{-+}$, is energy dependent and absorbs the last term in the brackets in the expression for $G_{d}^{A}(\omega)$. However, what still holds, even in the energy-independent case, is the relation $G^{+-}-G^{-+}=-2 \pi i \rho_{d}(\omega)$ and this is the reason why we have adopted $G_{d}^{+-}(\omega)-G_{d}^{-+}(\omega)$.

Before concluding this section we compare and discuss the expressions of $\Im m\left[G_{d}^{R}(\omega)\right]$ and $G_{d}^{+-}(\omega)-G_{d}^{-+}(\omega)$.

$$
\begin{gathered}
\Im m\left[G_{d}^{R}(\omega)\right]=\frac{\left[\Gamma+\Im m \Sigma^{--}-\Im m \Sigma^{-+}\right]}{\tilde{\Delta}(\omega)}, \\
G_{d}^{+-}(\omega)-G_{d}^{-+}(\omega)=\frac{\left[\Gamma-\Im m \Sigma^{-+}\right]}{\tilde{\Delta}(\omega)} .
\end{gathered}
$$

From the last two equations we observe that the difference between them lies in the term $\Im m \Sigma^{--}$. In non-equilibrium, for not too large Coulomb interactions $(U / \Gamma \approx 5)$ the imaginary part of $\Sigma^{--}$is negligible compared to $\Gamma$ and to $\Im m \Sigma^{-+}$, therefore we could still write the Meir Wingreen formula in its original form. In any case, for generality reasons we prefer to keep Eq. (4.33). Switching off the bias, but staying at $T=0$, we obtain $\Im m \Sigma^{-+}, \Im m \Sigma^{--} \rightarrow 0$ and thus recover exactly

$$
G_{d}^{A}(\omega)-G_{d}^{R}(\omega)=2 \pi i \rho_{d}(\omega)
$$

In passing we note that, even in an equilibrium situation (see Chapter 6 for a detailed illustration), but at finite temperature $T \neq 0$ the imaginary parts $\Im m \Sigma^{-+}, \Im m \Sigma^{--}$ are not zero, resulting again in the breaking of Eq. (4.35).

Another quantity of interest, which will be discussed more in detail in the following is $\Delta J=J_{L}-J_{R}$ [33]. Since no charge is produced on the quantum dot, $\Delta J=0$ in the exact solution. Using again the results of Meir-Wingreen [63] and Wingreen and Meir [89], the expression for $\Delta J$ becomes 


$$
\Delta J=-\frac{\Gamma}{\pi} \int_{-\infty}^{\infty} d \omega \frac{F(\omega)\left[\Im m \Sigma^{-+}(\omega)-\Im m \Sigma^{+-}(\omega)\right]-2 \Im m \Sigma^{-+}(\omega)}{\tilde{\Delta}(\omega)}
$$

Depending on the type of approximation used $\Delta J=0$ might either hold for all parameters [84, 89] or not [33]. We note that fulfilling $\Delta J=0$ is, however, not sufficient for an approximation to provide reliable results. E.g. the self-consistent Hartree-Fock approximation fulfills $\Delta J=0$, but nonetheless does not capture the correct physics even in equilibrium [34].

\subsection{Lowest order approximation}

Before studying the coupled system for $\hat{\Sigma}^{\Lambda}$ and $\gamma_{2}^{\Lambda}$ we begin with the simpler case where we replace $\gamma_{2}^{\Lambda}$ on the right hand side of Eq. (4.22) by the antisymmetrized bare interaction and consider only the flow of $\hat{\Sigma}$. With this replacement Eq. (4.22) reduces to

$$
\frac{d}{d \Lambda} \Sigma^{\mp \mp, \Lambda}= \pm i U \int \frac{d \omega}{2 \pi} \mathcal{S}^{\mp \mp, \Lambda}(\omega)
$$

Within this approximation the selfenergy is always time or frequency independent and no off-diagonal terms, in the Keldysh contour indices, are generated. It leads to, at least qualitatively, good results in equilibrium [2], where the flow equations can in addition be solved analytically. With $\hat{\Sigma}^{\Lambda}$ being diagonal in the Keldysh indices, a straightforward calculation permits us thus to rewrite Eq. (4.40) as

$$
\frac{d}{d \Lambda} \Sigma^{\mp \mp, \Lambda}= \pm \frac{i U}{2 \pi} \sum_{\omega= \pm \Lambda} \frac{\frac{G_{d, 0}^{\mp \mp}(\omega)}{\Delta(\omega)}-\Sigma^{ \pm \pm, \Lambda}}{\left(\frac{G_{d, 0}^{++}(\omega)}{\Delta(\omega)}-\Sigma^{--, \Lambda}\right)\left(\frac{G_{d, 0}^{--}(\omega)}{\Delta(\omega)}-\Sigma^{++, \Lambda}\right)-\left(\frac{G_{d, 0}^{-+}(\omega) G_{d, 0}^{+-}(\omega)}{\Delta(\omega)^{2}}\right)},
$$

where

$$
\begin{aligned}
\Delta(\omega) & =G_{d, 0}^{--}(\omega) G_{d, 0}^{++}(\omega)-G_{d, 0}^{-+}(\omega) G_{d, 0}^{+-}(\omega) \\
& =-\frac{1}{\left(\omega-V_{G}\right)^{2}+\Gamma^{2}} .
\end{aligned}
$$

\section{Equilibrium}

We now focus on $T=0$. In a first step we discuss the equilibrium situation, that is $V_{B}=\mu_{L}-\mu_{R} \rightarrow 0$. Then we obtain the decoupled system

$$
\frac{d}{d \Lambda} \Sigma^{\mp \mp, \Lambda}=i \frac{U}{\pi} \frac{V_{G} \pm \Sigma^{\mp \mp, \Lambda}}{\left[(\Lambda \pm i \Gamma)^{2}-\left(V_{G} \pm \Sigma^{\mp \mp, \Lambda}\right)^{2}\right]},
$$


which can be solved analytically. We first note that with $\Sigma^{++, \Lambda}=-\left[\Sigma^{--, \Lambda}\right]^{*}$ both equations are equivalent. For $\Sigma^{--, \Lambda}$ we obtain with the definition $\sigma^{\Lambda}=V_{G}+\Sigma^{--, \Lambda}$ the solution

$$
\frac{i \sigma^{\Lambda} J_{1}\left(\frac{\pi \sigma^{\Lambda}}{U}\right)-(\Lambda+i \Gamma) J_{0}\left(\frac{\pi \sigma^{\Lambda}}{U}\right)}{i \sigma^{\Lambda} Y_{1}\left(\frac{\pi \sigma^{\Lambda}}{U}\right)-(\Lambda+i \Gamma) Y_{0}\left(\frac{\pi \sigma^{\Lambda}}{U}\right)}=\frac{J_{0}\left(\frac{\pi V_{G}}{U}\right)}{Y_{0}\left(\frac{\pi V_{G}}{U}\right)},
$$

where $J_{n}$ and $Y_{n}$ are the Bessel functions of first and second kind. The desired solution of the cutoff free problem is obtained by setting $\Lambda=0$, i.e.

$$
\frac{\sigma J_{1}\left(\frac{\pi \sigma}{U}\right)-\Gamma J_{0}\left(\frac{\pi \sigma}{U}\right)}{\sigma Y_{1}\left(\frac{\pi \sigma}{U}\right)-\Gamma Y_{0}\left(\frac{\pi \sigma}{U}\right)}=\frac{J_{0}\left(\frac{\pi V_{G}}{U}\right)}{Y_{0}\left(\frac{\pi V_{G}}{U}\right)} .
$$

which is precisely the result Eq. (4) obtained by Karrasch et al. [2]. It is, however, important to note that in the imaginary-time formulation of the fRG, the differential equation has a different structure. It is real and has a positive definite denominator. Thus, while the solutions at $\Lambda=0$ are identical for the imaginary-time and real-time formulations, the flow towards $\Lambda=0$ will show differences. As we will see next, the complex nature of the differential equation (4.42) can lead to problems connected to its analytical structure when attempting a numerical solution. For small $U / \Gamma$ no particular problems arise. As an example the result for the flow of $\Sigma^{--, \Lambda}$ as function of $\Lambda$ for $U / \Gamma=1$ and $V_{G} / \Gamma=0.5$ obtained with a standard Runge-Kutta solver is shown in Fig. 4.2. Consistent with the analytical solution Eq. (4.44), the imaginary part (dashed line) goes to zero as $\Lambda \rightarrow 0$, while the real part (solid line) rapidly approaches the value given by formula Eq.(4.44).

However, for larger values of $U / \Gamma$ the numerical solution becomes unstable in a certain regime of $V_{G}$. A typical result in such a situation is shown in Fig. 4.3. The different curves were obtained as follows: The full and dashed ones from the numerical solution starting with $\Sigma^{--, \Lambda_{0}}=0$ at $\Lambda_{0} \rightarrow \infty$, the dash-dotted and dotted by integrating the differential equation (4.42) backwards from $\Lambda=0$ with the correct solution for $\Lambda=0$ as given by formula Eq. (4.44) as initial value. The crosses finally are the results from the analytical solution Eq. (4.43). Evidently, there exists a crossing of different branches of solutions to the differential equation for $\Lambda / \Gamma \approx 1$ and the numerical solution with starting point $\Lambda=\infty$ picks the wrong one as $\Lambda \rightarrow 0$. The reason for this behavior is that for large $U$ there exists a certain $V_{G}^{a}$ such that $V_{G}^{a}+\Sigma^{--, \Lambda_{p}}=\Lambda_{p}+i \Gamma$ with real $\Lambda_{p}$, resulting in a pole in the differential equation (4.42). For $V_{G} \neq V_{G}^{a}$ this pole does not appear for real $\Lambda_{p}$, but as shown in Fig. 4.4, $\Im m \Lambda_{p}$ changes sign at $V_{G}^{a}$, which in turn induces a sign change on the right hand side of the differential equation, leading to the behavior observed in Fig. 4.3. There also exists a second critical value $V_{G}^{b}$ such that for $V_{G}>V_{G}^{b}$ we find $\Im m \Lambda_{p}<0$ and the instability has vanished again. Obviously, this instability, which leads to problems in the numerical solution, limits the applicability of the present approximation to sufficiently small values of $U$. This is different from the imaginary-time approach by 


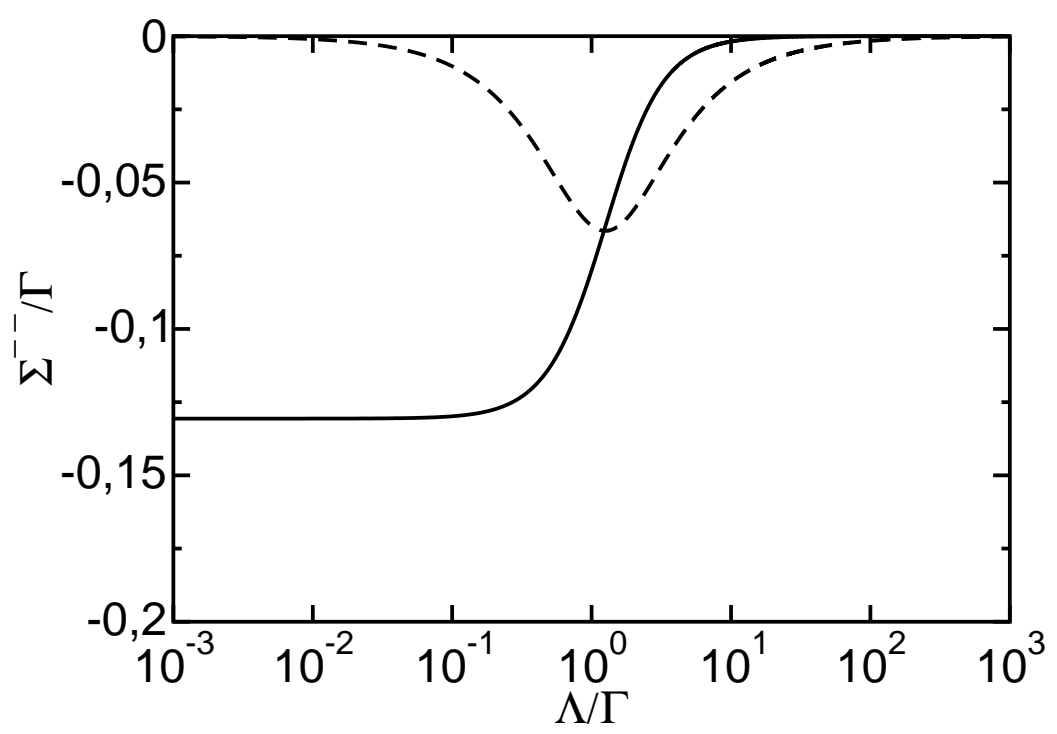

Figure 4.2: Flow of $\Sigma^{--, \Lambda} / \Gamma$ with $\Lambda / \Gamma$ for $U / \Gamma=1, V_{G} / \Gamma=0.5$, and $V_{B}=0$. The full curve shows the real part, the dashed the imaginary part of $\Sigma^{--, \Lambda}$.

Andergassen et al.,[2] where this simple approximation leads to qualitative correct results even for values of $U$ significantly larger than $\Gamma$.

\subsubsection{Non-equilibrium}

We now turn to the case of finite bias voltage $V_{B}$. As a typical example, the flow of $\Sigma^{--, \Lambda}$ for $U / \Gamma=1$ (full and dashed curves) and 5 (dashed-dotted and dotted curves) for $V_{G} / \Gamma=0.5$ at $V_{B} / \Gamma=0$ (equilibrium) and $V_{B} / \Gamma=1$ is shown in Fig. 4.5. Since the results for $\Sigma^{++, \Lambda}$ are related to those for $\Sigma^{--, \Lambda}$ by $\Sigma^{++, \Lambda}=-\left[\Sigma^{--, \Lambda}\right]^{*}$ we do not show them here. The $V_{B}$ dependence of the curves for $\Re e\left[\Sigma^{--, \Lambda}\right]$ (thick lines) looks sensible. For $V_{B} \neq 0$ an imaginary part of order $U^{2}$ is generated in the flow which does not vanish for $\Lambda \rightarrow 0$ (see the thin dotted line). Causality requires that the relation [52]

$$
\Sigma^{--}(\omega)+\Sigma^{++}(\omega)=-\left[\Sigma^{-+}(\omega)+\Sigma^{+-}(\omega)\right]
$$

must hold for the exact solution. Because of $\Sigma^{-+}(\omega)=\Sigma^{+-}(\omega)=0$, the finite imaginary part of $\Sigma^{\alpha \alpha}$ leads to a breaking of the condition Eq.(4.45) to order $U^{2}$ at the end of the FRG flow. This is consistent with the fact that by neglecting the flow of the vertex terms of order $U^{2}$ are only partially kept in the present FRG truncation scheme. The weak breaking of causality can also be understood as a consequence of our approximation leading to a complex, energy-independent self-energy: The offdiagonal components, being related to the distribution functions for electrons and 


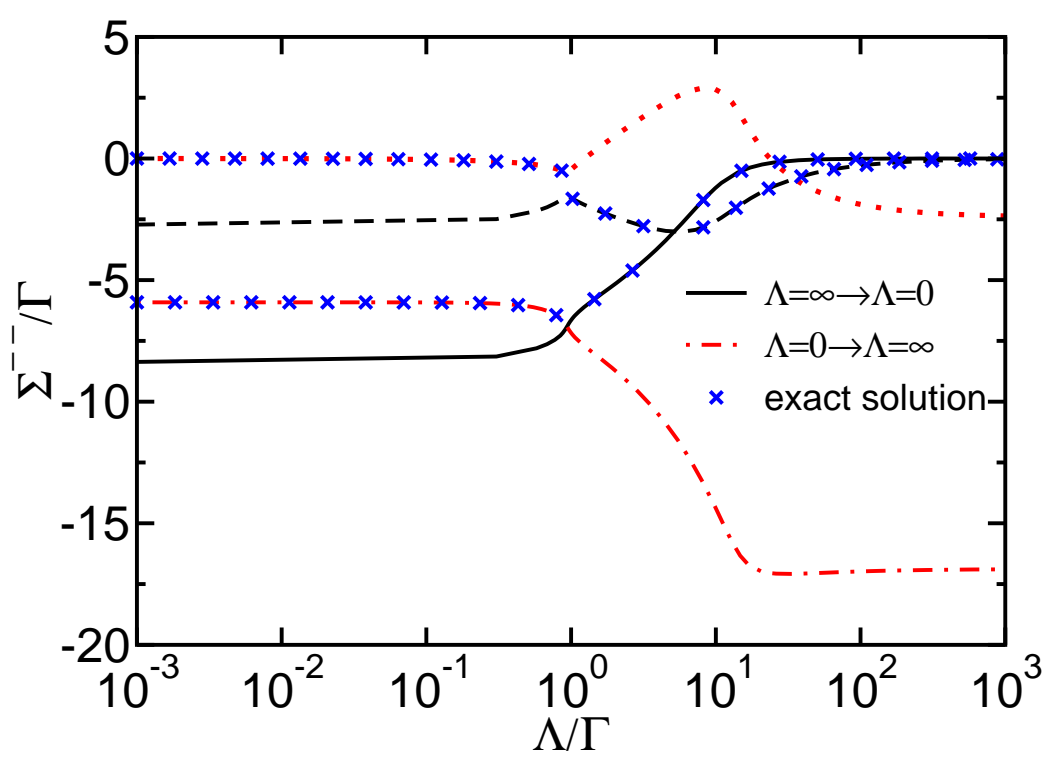

Figure 4.3: Flow of $\Sigma^{--, \Lambda} / \Gamma$ with $\Lambda / \Gamma$ for $U / \Gamma=15, V_{G} / \Gamma=6$, and $V_{B}=0$. The full and dashed curves show real and imaginary part obtained from the integration $\Lambda=\infty \rightarrow \Lambda=0$, the dashed-dotted and dotted curves real and imaginary part obtained from an integration $\Lambda=0 \rightarrow \Lambda=\infty$, using the solution from (4.44) as initial value for $\Sigma^{--, \Lambda}$. The crosses denote the analytical solution (4.43).

holes, respectively, in general have different support on the energy axis. The energy independence makes it impossible to respect this structure here.

For our further discussion the order $U^{2}$ violation of Eq.(4.45) means that we may not rely on relations like Eqs. (4.35) but have to work with $G^{\alpha \beta}$, thus the somewhat unusual formula (4.33). A naive application of $\Sigma^{R}=\Sigma^{--}-\Sigma^{-+}$and use of $G_{d}^{+-}-G_{d}^{-+}=2 i \Im m G_{d}^{R}$ would have led to unphysical results. That working with $G_{d}^{+-}-G_{d}^{-+}$is still sensible can be seen from a straightforward evaluation leading to

$$
\begin{aligned}
& G_{d}^{+-}(\omega)-G_{d}^{-+}(\omega)= \\
& -2 i \frac{\Gamma}{\left|\omega-V_{G}+i \Gamma[1-F(\omega)]-\Sigma^{--}\right|^{2}+\Gamma^{2} F(\omega) F(-\omega)} \\
& F(\omega)=f_{L}(\omega)+f_{R}(\omega)
\end{aligned}
$$

which is purely imaginary with a definite sign. Inserting the expression Eq. (4.46) into the formula Eq. (4.33), one can calculate the current and thus the conductance. Since we are at $T=0$, an explicit expression for the current of the cutoff free problem (at $\Lambda=0$ ) can be obtained by noting that with $\mu_{L}=V_{B} / 2, \mu_{R}=-V_{B} / 2$ one has $f_{L}(\omega)-f_{R}(\omega)=\Theta\left(V_{B} / 2-|\omega|\right)$ and $F( \pm \omega)=1$ for $\omega \in\left[-V_{B} / 2, V_{B} / 2\right]$, which leads 


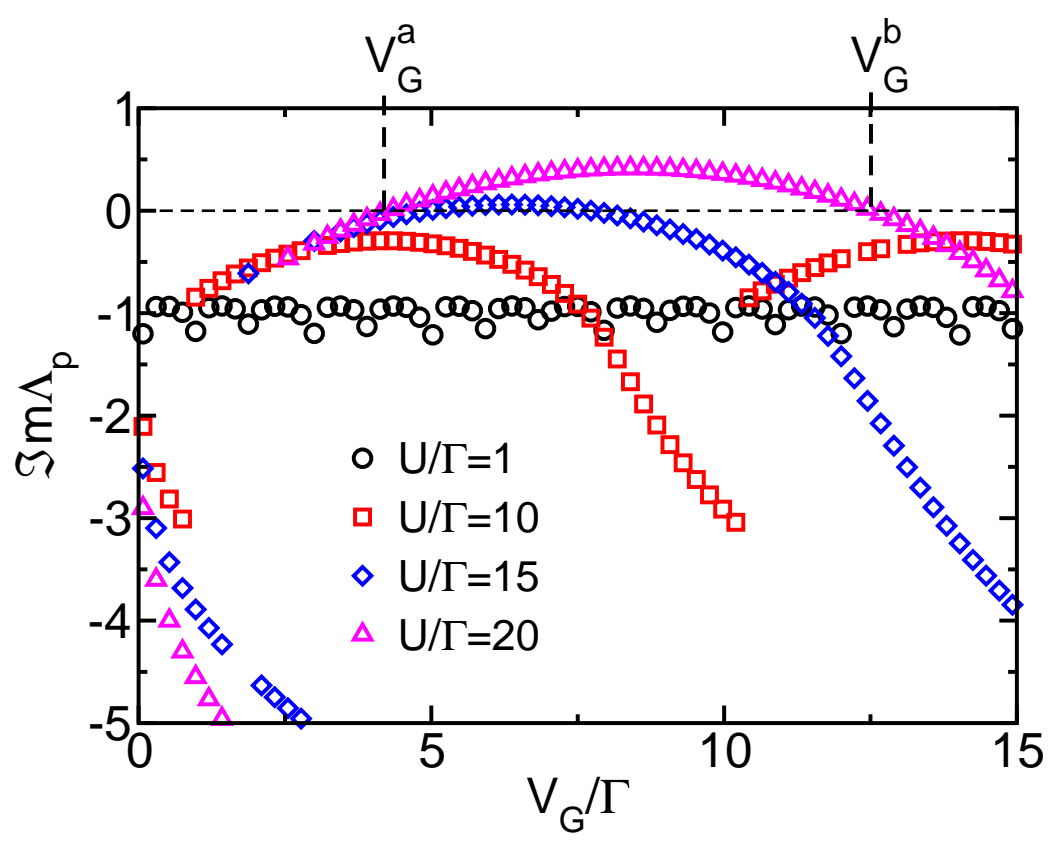

Figure 4.4: Imaginary part of $\Lambda_{p}$ determined from (4.43) and $V_{G}+\Sigma^{--, \Lambda_{p}}=\Lambda_{p}+i \Gamma$ for different values of $U$ and $V_{B}=0$ as function of $V_{G}$. For $U / \Gamma=15$ and 20 there exist an interval $\left[V_{G}^{a}, V_{G}^{b}\right]$ where $\Im m \Lambda_{p}>0$, while for small $U$ or $V_{G} \notin\left[V_{G}^{a}, V_{G}^{b}\right]$ we always have $\Im m \Lambda_{p}<0$.

to

$$
\begin{aligned}
J & =\frac{\Gamma^{2}}{\pi} \int_{-V_{B} / 2}^{V_{B} / 2} d \omega \frac{1}{\left|\omega-V_{G}-\Sigma^{--}\right|^{2}+\Gamma^{2}} \\
& =\frac{\Gamma}{\pi} \frac{\Gamma}{\Gamma^{*}} \sum_{s= \pm 1} s \arctan \left(\frac{V_{G}^{*}+s \frac{V_{B}}{2}}{\Gamma^{*}}\right)
\end{aligned}
$$

with the abbreviations

$$
\begin{aligned}
V_{G}^{*} & =V_{G}+\Re e \Sigma^{--}, \\
\Gamma^{*} & =\sqrt{\Gamma^{2}+\left(\Im m \Sigma^{--}\right)^{2}} .
\end{aligned}
$$

Equation (4.47) for the current is equivalent to the noninteracting expression but with renormalized parameters $V_{G}^{*}$ and $\Gamma^{*}$, which depend on the interaction as well as the bias and gate voltage.

An example for the differential conductance as function of $V_{G}$ obtained from Eq. (4.47) for $U / \Gamma=2$ and several values of $V_{B}$ is shown in Fig. 4.6, where $G_{0}=2 e^{2} / h$ 


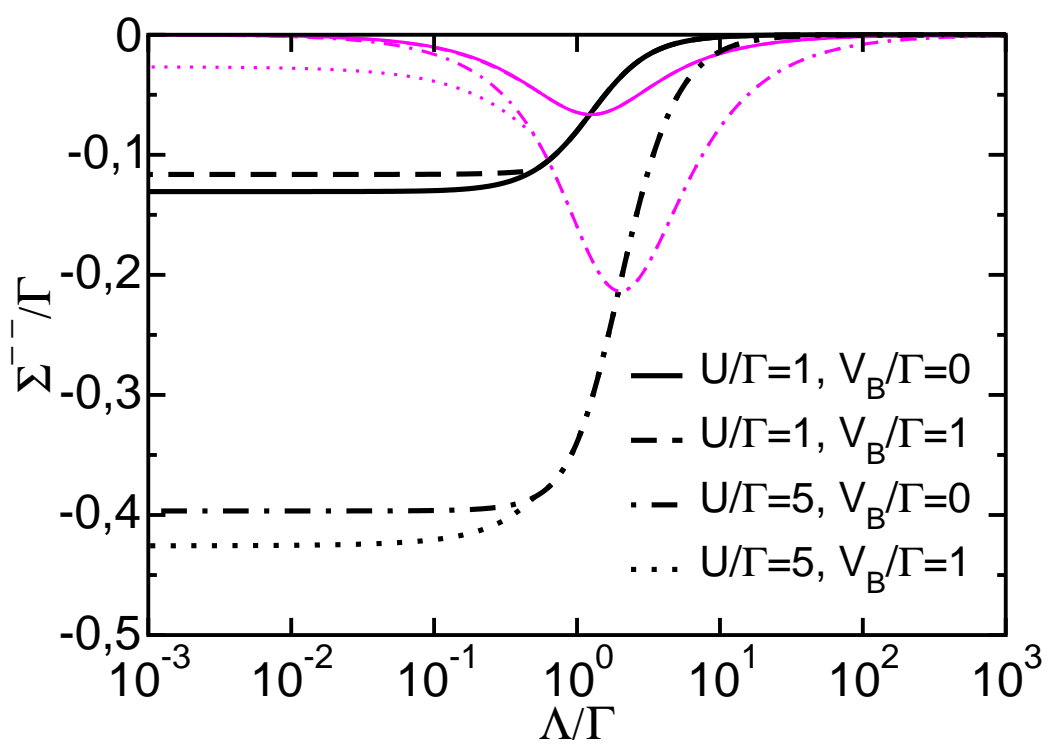

Figure 4.5: Flow of $\Sigma^{--, \Lambda} / \Gamma$ with $\Lambda$ for $U / \Gamma=1$ and 5 for $V_{G} / \Gamma=0.5$ at $V_{B} / \Gamma=0$ and 1 (thick curves: real part; thin curves: imaginary part). The curve for $\Im m \Sigma^{--, \Lambda} / \Gamma$ at $U / \Gamma=1$ and $V_{B} / \Gamma=1$ (thin dashed line) lies on top of the corresponding zero bias curve and is thus not visible.

(after reintroducing $e$ and $\hbar$ ). Increasing $V_{B}$ leads, as expected, first to a decrease of the conductance close to $V_{G}=0$ and later to a splitting of order $V_{B}$. Since we will discuss a more refined scheme including parts of the flow of the two-particle vertex next, we do not intend to dwell too much on the results of this simplest approximation. We note in passing that for $V_{G}=0$ due to particle-hole symmetry we obtain from the differential equation (4.41) that $\Sigma^{--}=0$ independent of $U$. Consequently the current $J$ calculated via Eq. (4.47) and the conductance are independent of $U$, too, and given by the corresponding expressions for the noninteracting system. As we will see in the next section, this deficiency will be cured by the approximate inclusion of the vertex flow. In the present approximation the current conservation $\Delta J=0$ holds for all parameters as $\Sigma^{-+}=\Sigma^{+-}=0$ [cf. Eq.(4.39)].

\subsection{Flowing vertex}

A more refined approximation is obtained when we insert the flowing two-particle vertex $\gamma_{2}^{\Lambda}$ as given by expression Eq. (4.30) in the calculation of the self-energy Eq. (5.1). By this we introduce an energy-dependence of the self-energy [30]. However, because the size of the resulting system of differential equations becomes extremely large if the full frequency dependence is kept (for a discussion on this in equilibrium see Hedden et al. [30]), we only keep the flow of the frequency independent part 


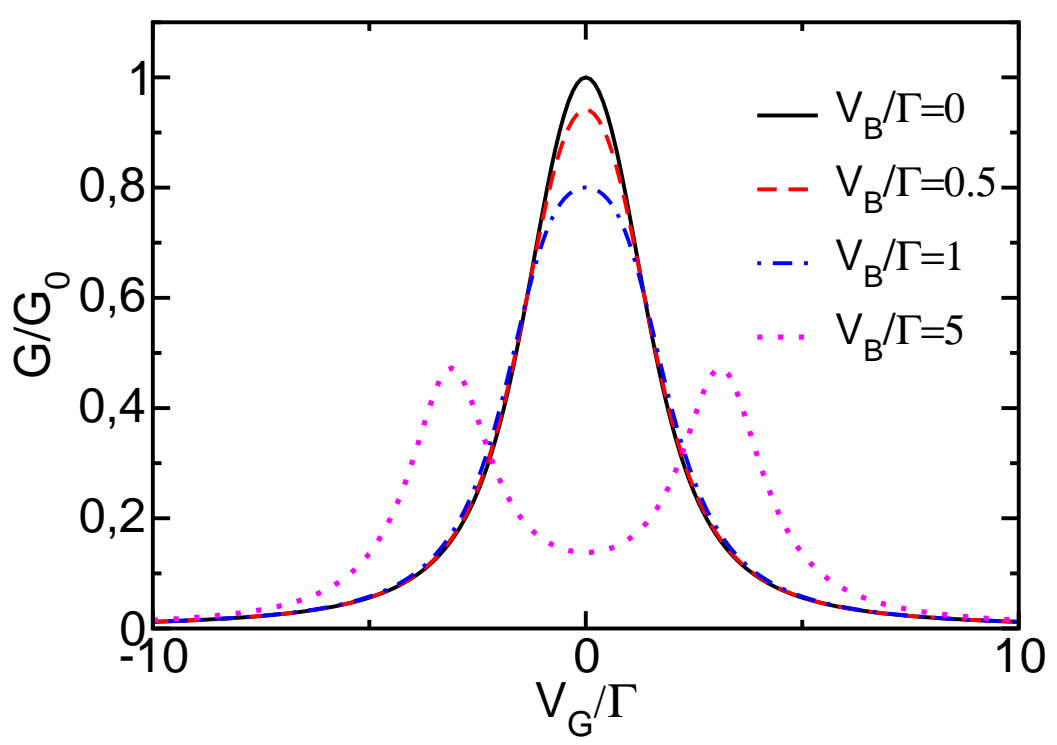

Figure 4.6: Conductance normalized to $G_{0}=2 e^{2} / h$ as function of $V_{G}$ for $U / \Gamma=2$ and several values of the bias voltage $V_{B}$.

of the vertex, an additional approximation which again has successfully been used in equilibrium [42]. As a consequence we again end up with a frequency independent $\hat{\Sigma}^{\Lambda}$. The resulting expression for the self-energy (see Section 4.3) is given by Eq. (4.29). This approximation leads to a surprisingly accurate description of the transport properties in equilibrium. In particular it is superior to the lowest order approximation including only the bare vertex.

\section{Equilibrium}

We again begin with the discussion of the solution to Eq. (4.29) in equilibrium. Results for the flow of $\Sigma^{--, \Lambda}$ are presented in Fig. 4.7 for $U / \Gamma=1$ (left panel) and $U / \Gamma=15$ (right panel) for $V_{G}=U / 2$. Since $\Sigma^{++, \Lambda}=-\left[\Sigma^{--, \Lambda}\right]^{*}$ only one component is shown. The stars in Fig. 4.7 denote the solutions of the imaginarytime equations taken from Karrasch et al. [42]. Note that for $U / \Gamma=15$ and $V_{G} / \Gamma>6$ the simple approximation Eq.(4.41) showed an instability, while with the flowing vertex the system is stable even for these large values of $U$ and reproduces the correct equilibrium solutions for $\Lambda \rightarrow 0$ [42]. The reason for this is that the flow of the vertex reduces the resulting effective interaction below the critical value in the instability region [42]. 

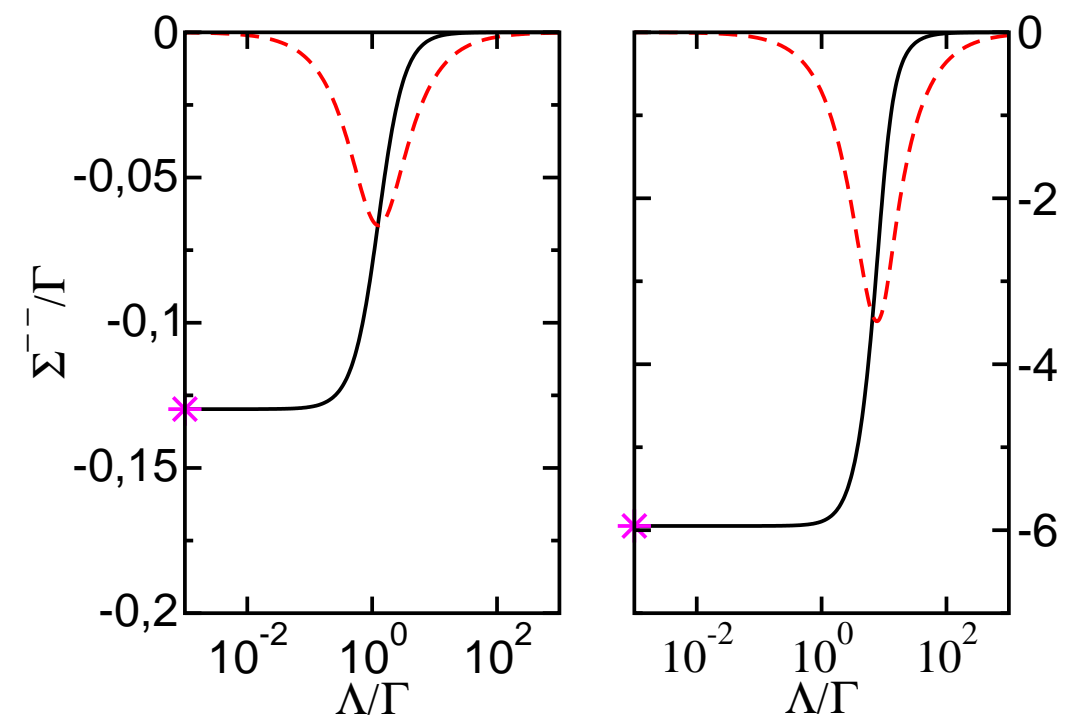

Figure 4.7: Flow of $\Sigma^{--, \Lambda} / \Gamma$ with $\Lambda$ for $U / \Gamma=1$ (left panel) and $U / \Gamma=15$ (right panel) at $V_{G}=U / 2$ and $V_{B}=0$. The full curves show the real part, the dashed the imaginary part of $\Sigma^{--, \Lambda}$. The stars at the vertical axis denote the values as obtained from the imaginary-time FRG [42]

\section{Nonequilibrium}

For the same parameters as in Fig. 4.7 we present the resulting flow with finite bias $V_{B} / \Gamma=1$ in Fig. 4.8. In addition to the curves for real (solid lines) and imaginary part (dashed lines) of $\Sigma^{--}$a third curve is displayed, the imaginary part of $\Sigma^{-+}$(dashed-dotted lines), which now is generated during the flow. Note that $\Re e \Sigma^{-+, \Lambda}=0$ and $\Sigma^{+-, \Lambda}=\left[\Sigma^{-+, \Lambda}\right]^{*}$. Furthermore, we always find $\Im m \Sigma^{-+, \Lambda}<0$. For $U / \Gamma=15$ (right panel in Fig. 4.8) we have rescaled $\Im m \Sigma^{-+, \Lambda}$ by a factor $10^{2}$ to make it visible on the scale of $\Sigma^{--, \Lambda}$. Since $\Sigma^{\alpha \beta, \Lambda}$ is a complex energy independent quantity Eq. (4.45) is again not fulfilled. We note that the error is still of order $U^{2}$, but for fixed $V_{G}$ and $V_{B}$ it is significantly smaller than in the simplest truncation scheme discussed above. The energy-independence of the self-energy allows to derive an analytical expression for the current at $T=0$ similar to Eq.(4.47), which due to the appearance of $\Sigma^{-+}$now becomes

$$
J=\frac{\Gamma}{\pi} \frac{\tilde{\Gamma}}{\Gamma^{*}} \sum_{s= \pm 1} s \arctan \left(\frac{V_{G}^{*}+s \frac{V_{B}}{2}}{\Gamma^{*}}\right)
$$



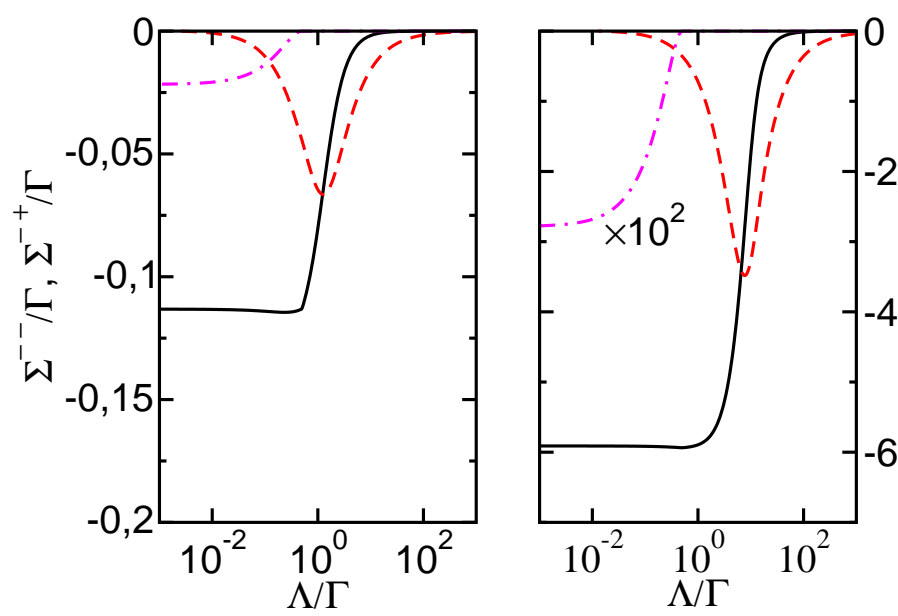

Figure 4.8: Flow of $\Sigma^{--, \Lambda} / \Gamma$ and $\Sigma^{-+, \Lambda} / \Gamma$ with $\Lambda$ for $U / \Gamma=1$ (left panel) and $U / \Gamma=15$ (right panel), $V_{G} / \Gamma=U / 2$ and $V_{B} / \Gamma=1$. The full curves show the real part, the dashed the imaginary part of $\Sigma^{--, \Lambda}$, the dot-dashed the imaginary part of $\Sigma^{-+, \Lambda}$. The real part for the latter is zero.

with $V_{G}^{*}$ as in Eq. (4.48) and ${ }^{1}$

$$
\begin{gathered}
\tilde{\Gamma}=\Gamma-\Im m \Sigma^{-+}>\Gamma \\
\Gamma^{*}=\sqrt{\tilde{\Gamma}^{2}+\left(\Im m \Sigma^{--}\right)^{2}} .
\end{gathered}
$$

where $\Sigma^{\alpha \beta}$ is taken at $\Lambda=0$. Thus, the only change to the expression (4.47) is a formal replacement $\Gamma \rightarrow \tilde{\Gamma}$ in $J / \Gamma$. Equation (4.50) is of the same structure as for the noninteracting case with $V_{G}$ and $\Gamma$ replaced by renormalized parameters. However, the two self-energy contributions $\Sigma^{--}$and $\Sigma^{-+}$enter distinctively different in the expression for the current. While $\Im m \Sigma^{--}$solely plays the role of an additional lifetime broadening, $\Im m \Sigma^{-+}$directly modifies the tunneling rate both in the prefactor of $J$ and in the expression for the life-time broadening. A problem occurs when using the results of the present approximation in Eq. (4.39), leading to

$$
\begin{gathered}
\Delta J=\frac{2 \Gamma}{\pi} \int_{-\infty}^{\infty} d \omega \Im m \Sigma^{-+}[1-F(\omega)] \\
\frac{1}{\left|\omega-V_{G}+i \Gamma[1-F(\omega)]-\Sigma^{--}\right|^{2}+\left[\Gamma F(-\omega)+\Im m \Sigma^{+-}\right]\left[\Gamma F(\omega)-\Im m \Sigma^{-+}\right]}
\end{gathered}
$$

\footnotetext{
${ }^{1}$ We remember the reader that, in the Eq 4.51, $\Im m \Sigma^{-+} \neq 0$ only when we take into account the vertex contributions.
} 




Figure 4.9: The current difference $\Delta J$ is plotted versus $U / \Gamma$ for $V_{B} / \Gamma=5$ and $V_{G} / \Gamma=2$. The plot shows a quadtratic behaviour of $\Delta J$ as function of $U / \Gamma$.

The requirement $\Delta J=0$ is only fulfilled for $V_{G}=0$, because then $\Sigma^{--}=0$ and the integrand is asymmetric with respect to $\omega$. Thus our approximation for the energy-independent flowing vertex violates current conservation for $V_{G} \neq 0$ in nonequilibrium. We verified that $\Delta J \sim U^{2}$ (see Fig. 4.9) which is consistent with the fact that not all terms of order $U^{2}$ are kept in our truncated FRG procedure. How does $\Delta J$ behave in the limit $V_{B} \rightarrow 0$ ? To see this we note that, because $\Im m \Sigma^{-+}$does not depend on the sign of $V_{B}$ and furthermore goes to zero as $V_{B} \rightarrow 0$, $\Im m \Sigma^{-+} \stackrel{V_{B} \rightarrow 0}{\sim} V_{B}^{2}$ (see Fig. 4.10). Consequently, $\Delta J \stackrel{V_{B} \rightarrow 0}{\sim} V_{B}^{2}$ and hence the violation of current conservation vanishes in the linear response regime $V_{B} \rightarrow 0$.

In Fig. 4.11 we show the current at $V_{G}=0$ as function of $V_{B}$ for $U / \Gamma=1,6$ and 15. With increasing $U$ the current for intermediate $V_{B}$ is strongly suppressed. In addition there occurs a structure at low $V_{B}$, which turns into a region of negative differential conductance with increasing $U$. The appearance of such a shoulder in the current was observed in other calculations as well [16, 32, 33]. However, whether the negative differential conductance we find for still larger values of $U$ (c.f. Fig. 4.12 ) is a true feature of the model or rather an artifact of the approximations used is presently not clear and should be clarified in further investigations. However, negative differential conductance has also been observed in a slave-boson treatment of the model [83].

Keeping $V_{G}=0$ fixed, we can calculate the conductance $G=d J / d V_{B}$ as function of $V_{B}$ for different values of $U$. The results are collected in Fig. 4.12. In contrast to the simple approximation without flow of the vertex, the conductance is now 


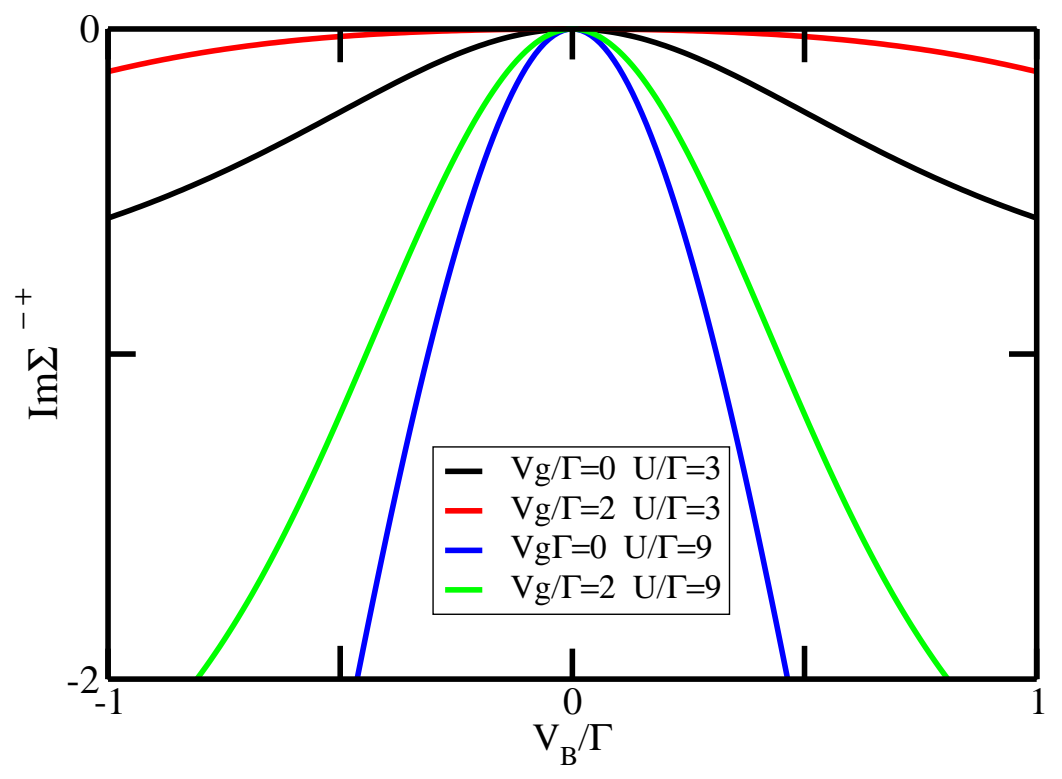

Figure 4.10: $\Im m \Sigma^{-+} / \Gamma$ as function of the bias voltage $V_{B} / \Gamma$ for several gate voltages $V_{G}$ and Coulomb interactions $U / \Gamma$. The plot shows a quadratic behaviour of $\Im m \Sigma^{-+} / \Gamma$ as function of the bias voltage which tends to zero in the limit $V_{B} / \Gamma \rightarrow 0$.

strongly dependent on $U$, except for $V_{B}=0$, where due to the unitary limit at $T=0$ we always find $G=G_{0}$. As already anticipated from the current in Fig. 4.11, a minimum in $G$ starts to form around $V_{B} / \Gamma \approx 0.5$ for $U / \Gamma>5$, which is accompanied by a peak at $V_{B} / \Gamma \approx 2$. A similar behavior in the conductance was observed in a perturbative treatment [16], which in contrast to our current approximation involves the full energy-dependence in the self-energy. This at least qualitative agreement - we of course cannot resolve structures like the Hubbard bands with an energy independent self-energy - again supports our claim that despite the violation of the relation Eq.(4.45) we can obtain reasonable results from $G^{\alpha \beta}$.

We finally discuss the variation of the conductance with $V_{G}$ for fixed $U$ and $V_{B}$. We again emphasize, that for $V_{G} \neq 0, \Delta J=0$ only holds to leading order in $U$. In Fig. 4.13 we present the curves for two different values of $U$, namely $U / \Gamma=1$ (upper panel in Fig. 4.13) and $U / \Gamma=15$ (lower panel in Fig. 4.13). In the former case, the variation of $G$ with $V_{B}$ is rather smooth, as is to be expected from the current in Fig. 4.11. For large $U$, we observe an extended plateau at zero bias, which is a manifestation of the fact that in the strong coupling regime a pinning of spectral weight at the Fermi energy occurs. This feature is also observed in the imaginary-time fRG as well as in NRG calculations. [42] Increasing $V_{B}$ quickly leads to a similarly extended region of negative differential conductance, which, assuming that this result is a true feature of the model, therefore seems to be linked to the 


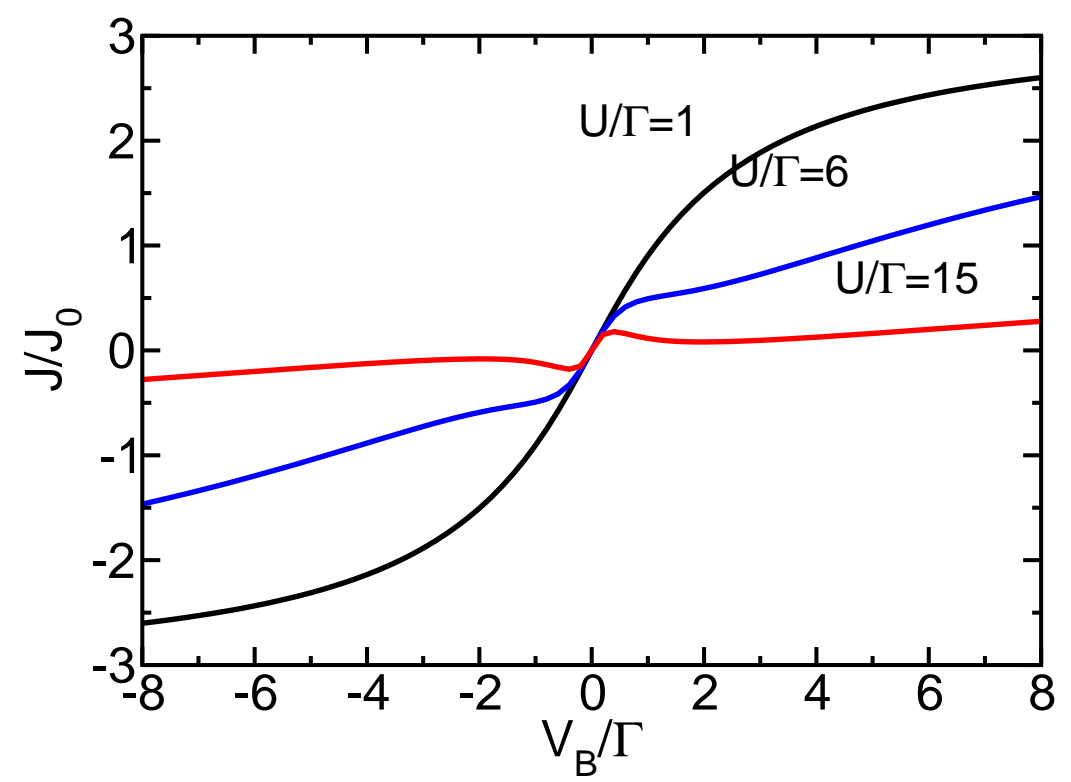

Figure 4.11: Current normalized to $J_{0}=G_{0} \frac{\Gamma}{e}$ (after reintroducing $e$ and $\hbar$ ) as function of $V_{B}$ for $U / \Gamma=1,6$ and 15 and $V_{G}=0$. For $U / \Gamma=15$ we find a region of negative differential conductance in the region $\left|V_{B} / \Gamma\right| \approx 0.5$ (c.f. Fig. 4.12).

"Kondo" pinning. We note that it is unlikely that the appearance of the negative differential conductance is related to the breaking of current conservation at order $U^{2}$ as it also appears for $V_{G}=0$ where $\Delta J=0$. For large $V_{B}$ multiple structures appear in $G$, which are related to the energy scales $V_{B}$ and $U$. 


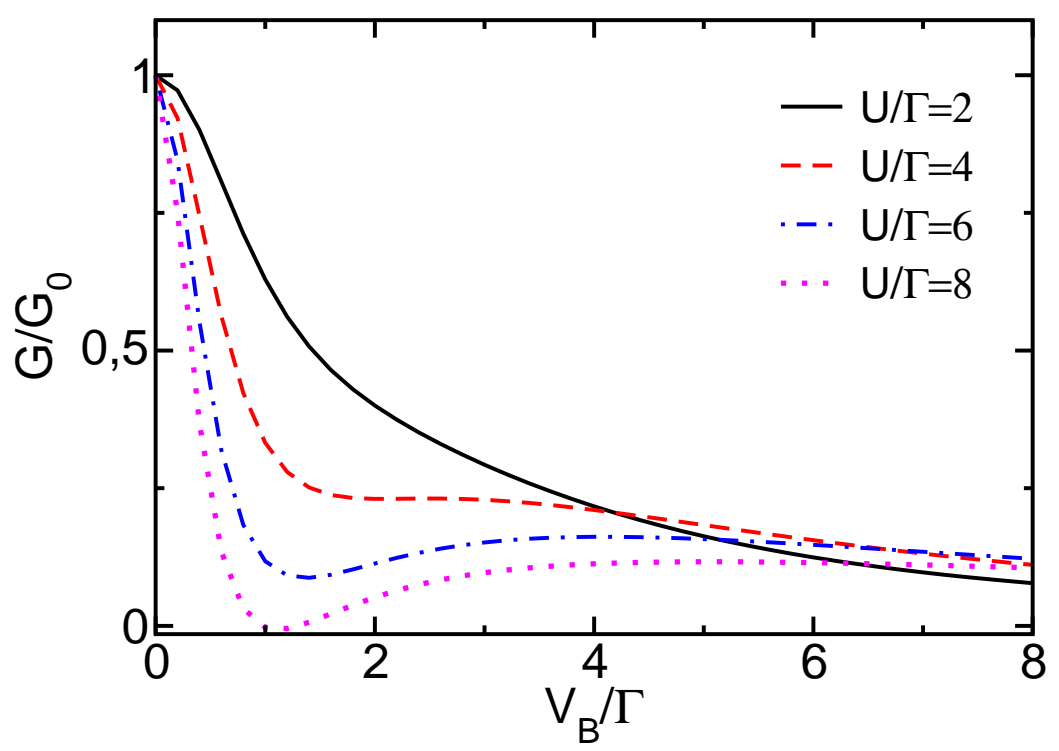

Figure 4.12: Differential conductance $G$ as function of $V_{B}$ for $V_{G}=0$ and various values of $U$. For $U / \Gamma>5$ a distinct minimum around $V_{B} / \Gamma \approx 0.5$ appears.



Figure 4.13: Differential conductance $G$ as function of $V_{G}$ for different values of $V_{B}$ and $U / \Gamma=1$ (upper panel) and $U / \Gamma=15$ (lower panel). Note the extended plateau at $V_{B}=0$ for $U / \Gamma=15$, which is a manifestation of the pinning of spectral weight at the Fermi level. 


\section{Transport Properties in a Magnetic Field}

\subsection{Introduction}

This section is dedicated to the analysis of the influence of an external magnetic field $B$ on the transport properties of a single-level quantum dot under a constant applied bias $V_{B}$ at $T=0$. We discuss how the current $J$ and the differential conductance $G$ are affected by $B$ and by the competition between magnetic field and bias voltage. We show in particular that $B$ is responsible for a switching behaviour in $J$ as function of $V_{B}$. Interesting are also the individual contributions of spin up and down electrons, split by the presence of the magnetic field, to the transport parameters. To test our non-equilibrium FRG we have again studied, as limiting case, the equilibrium situation $\left(V_{B}=0\right)$, in order to compare the results of the imaginary-time FRG [42]. In the next paragraph we will show the flow equations for the non-equilibrium FRG in an applied magnetic field, then we present the results. The equilibrium limit $V_{B}=0$ is shown and explained first, afterwards we discuss how the transport parameters $J, G$ behave as functions of the bias-voltage with magnetic field. Finally we consider the range of applicability of the non-equilibrium FRG in the presence of $B$.

\subsection{Flow Equations in a Magnetic Field}

A spin dependent quantum dot consisting of a single level $\varepsilon_{\sigma}$, coupled to left and right leads (whose electrons are non interacting and in equilibrium) through the energy independent terms $V_{\vec{k} \sigma \alpha}$, and subjected to Coulomb repulsion $U$, can be described by the Anderson Hamiltonian [4] (see Section. 1.4.2). The only difference with respect to the non magnetic case is that the magnetic field $B$ splits the dot level in its "up" and "down" components

$$
\varepsilon_{\uparrow,(\downarrow)}=V_{G} \pm B / 2
$$

introducing de facto a spin-dependence of the dot level, which must be now taken into account as additional parameter. In Section. 4.3 we have already discussed the flow equations of a single-level quantum dot without magnetic field, therefore we 
show now only the changes due to the spin dependence in the flow equations. As in the previous chapter, in addition to the truncation of the hierarchy of differential equations, obtained in the FRG, we neglect the energy dependence of the selfenergy $\Sigma$ and the vertex function $\gamma_{2}$. The system of differential equations we are going to integrate is then given by

$$
\frac{d}{d \Lambda} \Sigma_{\sigma_{1}^{\prime}, \sigma_{1}}^{\alpha \beta, \Lambda}=-\frac{1}{2 \pi} \sum_{\omega= \pm \Lambda} \sum_{\sigma_{2}^{\prime}, \sigma_{2}} \sum_{\mu \nu} \mathcal{G}_{d}^{\mu \nu, \Lambda}(\omega) \gamma_{2, \sigma_{1}^{\prime}, \sigma_{2}^{\prime}, \sigma_{1}, \sigma_{2}}^{\alpha \nu \beta \mu, \Lambda}
$$

$$
\begin{array}{r}
\frac{d}{d \Lambda} \gamma_{\sigma_{1}^{\prime}, \sigma_{2}^{\prime} ; \sigma_{1}, \sigma_{2}}^{\alpha \beta \gamma \delta, \Lambda}=\frac{1}{4 \pi} \sum_{\omega= \pm \Lambda} \sum_{\sigma_{3}, \sigma_{4}} \sum_{\mu, \nu \rho, \eta}\left(\mathcal{G}_{\sigma_{3}, d}^{\rho \eta, \Lambda}(-\omega) \mathcal{G}_{\sigma_{4}, d}^{\nu \mu, \Lambda}(\omega) \gamma_{\sigma_{1}^{\prime}, \sigma_{2}^{\prime} ; \sigma_{3}, \sigma_{4}}^{\alpha \beta \rho, \Lambda} \gamma_{\sigma_{3}, \sigma_{4} ; \sigma_{1}, \sigma_{2}}^{\eta \mu \gamma \delta, \Lambda}\right. \\
-\mathcal{G}_{\sigma_{3}, d}^{\eta \rho, \Lambda}(\omega) \mathcal{G}_{\sigma_{4}, d}^{\nu \mu, \Lambda}(\omega)\left[\gamma_{\sigma_{1}^{\prime}, \sigma_{4} ; \sigma_{1}, \sigma_{3}}^{\alpha \mu \gamma \eta, \Lambda} \gamma_{\sigma_{3}, \sigma_{2}^{\prime} ; \sigma_{4}, \sigma_{2}}^{\rho \beta \nu \delta \delta, \Lambda}+\gamma_{\sigma_{1}^{\prime}, \sigma_{3} ; \sigma_{1}, \sigma_{4}}^{\alpha \rho \gamma \nu, \Lambda} \gamma_{\sigma_{4}, \sigma_{2}^{\prime} ; \sigma_{3}, \sigma_{2}}^{\mu \beta \eta \delta, \Lambda}\right. \\
\left.\left.-\gamma_{\sigma_{2}^{\prime}, \sigma_{4} ; \sigma_{1}, \sigma_{3}}^{\beta \mu \gamma \eta, \Lambda} \gamma_{\sigma_{3}, \sigma_{1}^{\prime} ; \sigma_{4}, \sigma_{2}}^{\rho \alpha \nu \delta, \Lambda}-\gamma_{\sigma_{2}^{\prime}, \sigma_{3} ; \sigma_{1}, \sigma_{4}}^{\beta \rho \gamma \nu, \Lambda} \gamma_{\sigma_{4}, \sigma_{1}^{\prime} ; \sigma_{3}, \sigma_{2}}^{\mu \alpha \eta \delta, \Lambda}\right]\right)
\end{array}
$$

In expressions (5.1) and (5.2) $\hat{\mathcal{G}}_{\sigma, d}$ is given by Eq. (4.28). The initial conditions for the system (5.1)-(5.2) have been already discussed in Section 4.3. Eq. (5.1) and (5.2), compared to system obtained in Section 4.3 (without the presence of a magnetic field), show a more complicated structure which manifests itself in a spin-dependent flow for the selfenergy and the vertex. As further step, we apply the same parametrization for the vertex which we have introduced in the previous chapter (see Eq. (4.25)) leading to the flow equations

$$
\frac{d}{d \Lambda} \Sigma_{\sigma}^{\alpha \beta, \Lambda}=-\frac{1}{2 \pi} \sum_{\omega= \pm \Lambda, \gamma, \delta}\left(\mathcal{G}_{d, \sigma}^{\gamma \delta, \Lambda}(\omega)+\mathcal{G}_{d, \bar{\sigma}}^{\gamma \delta, \Lambda}(\omega)\right) U^{\alpha \beta \gamma \delta, \Lambda}-\mathcal{G}_{d, \sigma}^{\gamma \delta, \Lambda}(\omega) U^{\beta \alpha \gamma \delta, \Lambda}
$$




$$
\begin{array}{r}
\frac{d}{d \Lambda} U^{\alpha \beta \gamma \delta, \Lambda}=\frac{1}{4 \pi} \sum_{\omega= \pm \Lambda} \sum_{\mu, \nu \rho, \eta}\left(\mathcal{G}_{\bar{\sigma}}^{\rho \eta, \Lambda}(-\omega) \mathcal{G}_{\sigma}^{\nu \mu, \Lambda}(\omega)\left[U^{\alpha \beta \eta \nu, \Lambda} U^{\rho \mu \gamma \delta, \Lambda}\right]\right. \\
+\mathcal{G}_{\sigma}^{\rho \eta, \Lambda}(-\omega) \mathcal{G}_{\bar{\sigma}}^{\nu \mu, \Lambda}(\omega)\left[U^{\beta \alpha \eta \nu, \Lambda} U^{\mu \rho \gamma \delta, \Lambda}\right]- \\
{\left[\mathcal{G}_{\sigma}^{\eta \rho, \Lambda}(\omega) \mathcal{G}_{\sigma}^{\nu \mu, \Lambda}(\omega)+\mathcal{G}_{\bar{\sigma}}^{\eta \rho, \Lambda}(\omega) \mathcal{G}_{\bar{\sigma}}^{\nu \mu, \Lambda}(\omega)\right] U^{\alpha \mu \gamma \eta, \Lambda} U^{\rho \beta \nu \delta, \Lambda}-} \\
{\left[\mathcal{G}_{\sigma}^{\eta \rho, \Lambda}(\omega) \mathcal{G}_{\sigma}^{\nu \mu, \Lambda}(\omega)\right] U^{\alpha \mu \gamma \eta, \Lambda} U^{\beta \rho \nu \delta, \Lambda}-} \\
\\
{\left[\mathcal{G}_{\bar{\sigma}}^{\eta \rho, \Lambda}(\omega) \mathcal{G}_{\bar{\sigma}}^{\nu \mu, \Lambda}(\omega)\right] U^{\mu \alpha \gamma \eta, \Lambda} U^{\rho \beta \nu \delta, \Lambda}+} \\
{\left[\mathcal{G}_{\sigma}^{\eta \rho, \Lambda}(\omega) \mathcal{G}_{\sigma}^{\nu \mu, \Lambda}(\omega)+\mathcal{G}_{\bar{\sigma}}^{\eta \rho, \Lambda}(\omega) \mathcal{G}_{\bar{\sigma}}^{\nu \mu, \Lambda}(\omega)\right] U^{\alpha \rho \gamma \nu, \Lambda} U^{\mu \beta \eta \delta, \Lambda}-} \\
{\left[\mathcal{G}_{\sigma}^{\eta \rho, \Lambda}(\omega) \mathcal{G}_{\sigma}^{\nu \mu, \Lambda}(\omega)\right] U^{\alpha \rho \gamma \nu, \Lambda} U^{\beta \mu \eta \delta \delta, \Lambda}-} \\
{\left[\mathcal{G}_{\bar{\sigma}}^{\eta \rho, \Lambda}(\omega) \mathcal{G}_{\bar{\sigma}}^{\nu \mu, \Lambda}(\omega)\right] U^{\rho \alpha \gamma \nu, \Lambda} U^{\mu \beta \eta \delta, \Lambda}-} \\
\mathcal{G}_{\sigma}^{\eta \rho, \Lambda}(\omega) \mathcal{G}_{\bar{\sigma}}^{\nu \mu, \Lambda}(\omega)\left[U^{\mu \beta \gamma \eta, \Lambda} U^{\alpha \rho \nu \delta, \Lambda}\right]+ \\
\left.\mathcal{G}_{\bar{\sigma}}^{\eta \rho, \Lambda}(\omega) \mathcal{G}_{\sigma}^{\nu \mu, \Lambda}(\omega)\left[U^{\rho \beta \gamma \nu, \Lambda} U^{\alpha \mu \eta \delta, \Lambda}\right]\right)
\end{array}
$$

By investigating the vertex structure of the full flow at $\Lambda=0$ it turns out, however, that symmetries, implied by the parametrization, are broken for large voltages and magnetic fields. Due to the significantly reduced numerical effort for integrating Eq. (5.3) and Eq. (5.4), it remains interesting to examine its value as an approximation. Comparing the full flow according to Eqs. (5.1) and (5.2) with the parametrized one (Eqs. (5.3) and (5.4)) we observe a good agreement, at low $V_{B}$, for $\Sigma^{-+, \Lambda}$ (see Fig. 5.1 (b)), while deviations appear in $\Sigma^{--, \Lambda}$ (Fig. 5.1 (a)). However, since $\left|\Sigma^{-+, \Lambda}\right| \gg\left|\Sigma^{--, \Lambda}\right|$, this does not affect the behaviour of experimentally relevant quantities like conductance $G$ as function of the gate voltage, even for larger $B$ (see Fig. 5.2). Increasing the bias voltage, we obtain, for small $B$ (see Fig. 5.4, full curve and circles), again a good agreement.

As soon as we increase the magnetic field, $\operatorname{Im} \Sigma^{-+, \Lambda}$ shows deviations (see Fig. 5.3) which even affect numerical values and the shape of the conductance (see Fig. 5.4). In particular, the flow of the parametrized system gives rise to a small real component of $\Sigma^{+-, \Lambda}$ at $\Lambda=0$ (Fig. $5.3(\mathrm{~b})$ ), which actually should not exist, and leads to unphysical breaking of the particle-hole symmetry.

After integration of system Eqs. (5.1)-(5.2) or Eqs. (5.3)-(5.4) we insert the resulting selfenergy into the Meir-Wingreen formula for the current [19, 63]

$$
J_{\sigma}=\frac{i e \Gamma}{2 \pi \hbar} \int d \epsilon\left[f_{L}(\epsilon)-f_{R}(\epsilon)\right]\left(G_{d, \sigma}^{+-}(\epsilon)-G_{d, \sigma}^{-+}(\epsilon)\right) .
$$

In passing we observe that, with respect to the non-magnetic case, we have to substitute into the expressions of the free Green functions of the dot (Eqs. (4.18)- 




Figure 5.1: (a) Flow of real (full curve and stars) and imaginary part (dashed curve and crosses) of $\Sigma_{\downarrow}^{--, \Lambda} / \Gamma$, for $V_{G} / \Gamma=0, U / \Gamma=5, V_{B} / \Gamma=1, B / \Gamma=0.116$ The continuous lines represent the solutions of Eqs.(5.1),(4.30), the symbols the parametrized version Eqs.(4.29),(4.32). (b) Flow of $\Sigma_{\downarrow}^{-+, \Lambda} / \Gamma$. 


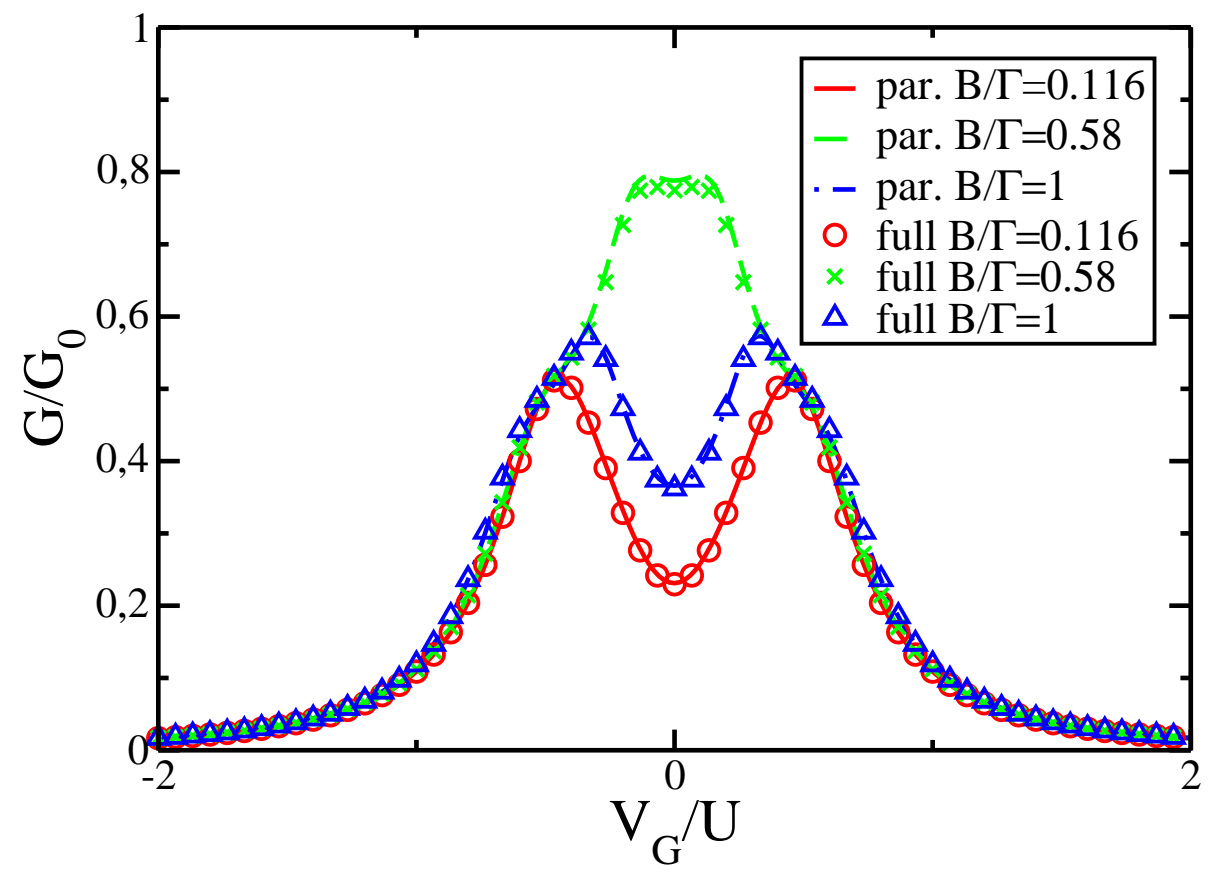

Figure 5.2: Total conductance $G=G_{\uparrow}+G_{\downarrow}$ normalized to $G_{0}=2 e^{2} / h$ as function of $V_{G}$ for $U / \Gamma=5, V_{B}=1$ and several values of $B / \Gamma$.



Figure 5.3: The same parameters range as in Fig.1 except for $V_{B} / \Gamma=3$ and $B / \Gamma=1$. In (b) the real part of $\Sigma^{-+}$has been rescaled by a factor ten to make it visible. 


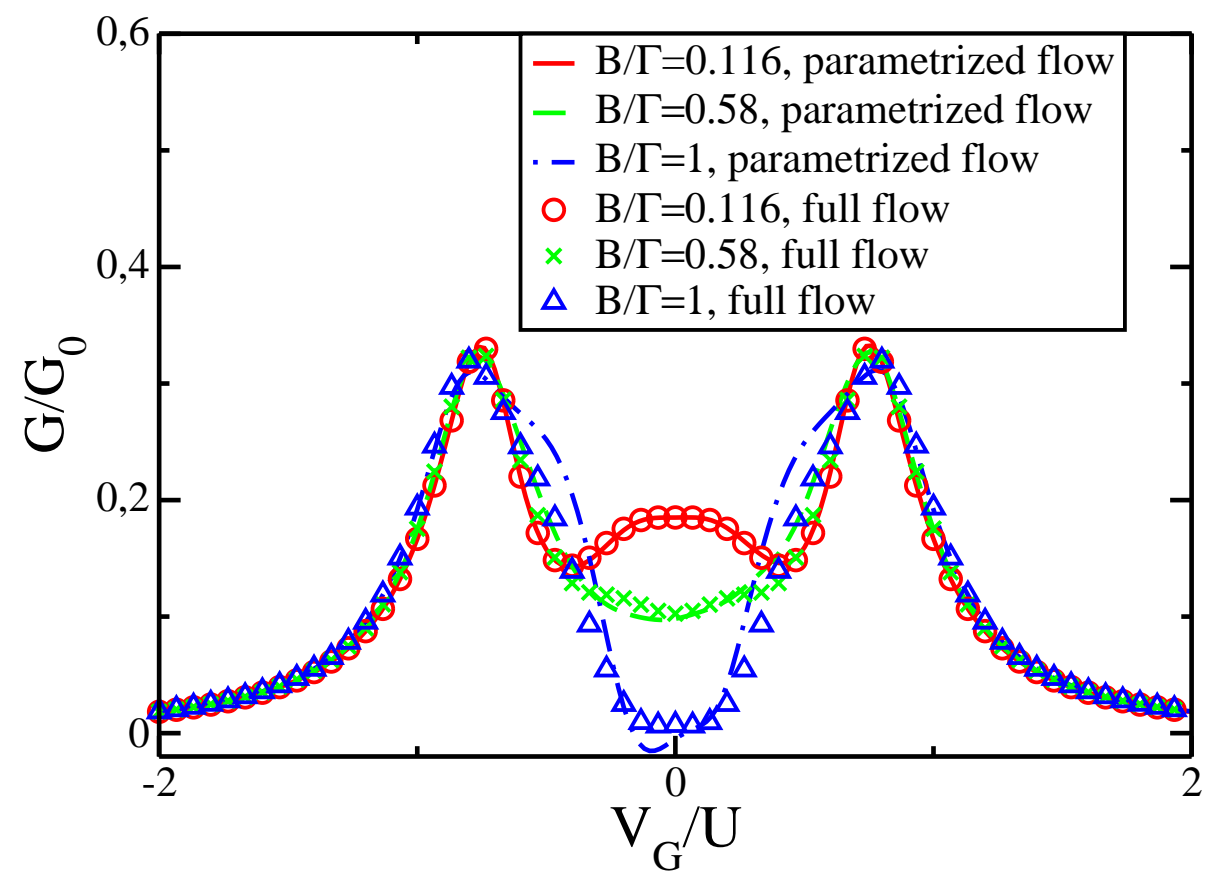

Figure 5.4: The same parameters range as in Fig.2 except for $V_{B} / \Gamma=3$

(4.21)) the bare dot level with the spin-dependent one

$$
\varepsilon \rightarrow \varepsilon_{\uparrow, \downarrow}=V_{G} \pm B / 2 \text {. }
$$

\subsection{Results}

\subsubsection{Equilibrium Case}

Before studying the stationary non-equilibrium case at $T=0$, we consider the equilibrium situation, namely the limit $V_{B}=\mu_{L}-\mu_{R}=0$. The latter can be seen as a test for our non-equilibrium FRG which should reproduce the imaginary-time FRG results [42]. Let us begin with the differential conductance $\mathrm{G}$ as function of the gate voltage $V_{G}$ with and without a magnetic field in the strong coupling regime $\frac{U}{\pi \Gamma}>1$. In Fig. 5.5 (a) we compare the total conductance $G=G_{\uparrow}+G_{\downarrow}$ for different values of $B$ with the results obtained by Karrasch et. al [42]. We note, that for $V_{B} / \Gamma=0$ our calculations perfectly reproduce the results of Karrasch et. al [42]. We see that, as soon as the magnetic field increases, $G$ starts to split in two peaks indicating the "up" and "down" contributions, which reflects the splitting in the spectral function. For an extensive discussion of the linear response results with an applied magnetic field we refer the reader to the seminal paper by Karrasch et. al [42]. As last remark 




Figure 5.5: Total conductance $G=G_{\uparrow}+G_{\downarrow}$ normalized to $G_{0}=2 e^{2} / h$ as function of $V_{G} / U$ for $U / \Gamma=5, V_{B} / \Gamma=0,1,3,5$ and several values of $B / \Gamma$. The black curves are obtained from the imaginary-time FRG.



Figure 5.6: Individual spin contributions to the total conductance in the same parameters regime as in Fig. 5.2 
we note that also the individual spin contribution agree perfectly with [42] as shown in Fig. 5.6.

\subsubsection{Non-Equilibrium}

Switching on the bias voltage $V_{B}$ we observe different behaviours of the conductance $G$ as a function of the gate voltage $V_{G}$ depending on the competition between the voltage and the magnetic field. In Fig. 5.5, we present $G$ as function of $V_{G}$ for a fixed Coulomb interaction $U / \Gamma=5$ and $V_{B} / \Gamma=0,1,3,5$ for different values of $B$. We observe a drastic change of the structure due to the interplay of $V_{B}$ and $B$. In particular, we see that for small bias $\left(V_{B} / \Gamma=1\right)$ and fields $(B / \Gamma=0.116), G$ consists of just two peaks separated by $U$. If we now increase the value of the magnetic field, the conductance, at small $V_{G}$, initially strongly increases (dashed and dot-dashed curves in Fig. 5.5 (b)), the peaks disappear and a small plateau appears. Increasing $B$ further, the plateau disappears and we get back the two peaks separated by a rather deep valley and two shoulders whose spacing is $\Delta \approx U+V_{B}$.

This behaviour can be explained as follows: The spectral density is split by $V_{B}$ in two peaks moving the spectral weight to higher frequencies and decreasing it in the region $\omega \approx 0$. Switching on $B$ we observe an additional split of each peak, due to the spin contributions. Increasing then $B$, the two external peaks move away from each other and the internal ones get closer and even merge enhancing the spectral weight at $\omega \approx 0$. As direct consequence we observe an enhancement of $G$, which manifests itself in a small plateau for $V_{G} / U \approx 0$ (see Fig. $5.5(\mathrm{~b})$ ). With a further increase of $B$, the "merged" peaks move away from each other, leading to a collapse of the conductance, and disappearance of the plateau. Completely different is the behaviour for $V_{B} / \Gamma=3$ and $V_{B} / \Gamma=5$ (Fig. 5.5 (c) and (d)). We find a nonmonotonic decrease of $\mathrm{G}$ with the magnetic field. In addition the field dependence is initially weaker than in Fig. 5.5 (b). We interpret this behaviour in the following way: For large $V_{B}$, the Fermi window in eg. Eq. (5.5) will lead to an averaging over a large energy region. Thus structures due to the magnetic field at too small energies will be washed out.

\subsubsection{Current and Conductance as Function of the Applied Bias}

The non-monotonic behaviour of $G\left(V_{G}=0\right)$ is an interesting feature we want to explore in somewhat more detail in the following. To this end we calculated the current $J$ and the differential conductance $G=d J / d V_{B}$, at $V_{G} / \Gamma=0$ and $U / \Gamma=5$, as function of the applied bias for different values of $B$. The results are collected in Fig. 5.7. Compared to the non-magnetic case (full line) we see that a finite magnetic field $B$ basically induces two features. First, as is also for the case for $B=0$, at large 




Figure 5.7: Total current (normalized to $J_{0}=G_{0} \frac{\Gamma}{e}$, with $G_{0}=2 e^{2} / h$ ) and conductance as function of the bias voltage $V_{B} / \Gamma$ for $V_{G} / \Gamma=0, U / \Gamma=5$, and several values of $B$

$U$, we observe a small region of negative differential conductance. More interesting is the appearence of an almost unitary conductance peak at $V_{B} \approx 2 B$ for intermediate fields. For large fields the features is suppressed again. Thus we observe that $B$ can act as a switch, increasing the current dramatically in the voltage range $V_{B} \approx B$. For $V_{B} \gg B$ the curves tend to the same values, so that $B$ does not influence the current any longer. This behaviour can be explained by noting that when $V_{B} \approx 2 B$, the electrochemical potentials $\mu_{L, R}$ of the leads are close to the split dot levels, respectively, therefore the tunnel probability from the leads to the dot is enhanced as long as the $V_{B}=\mu_{L}-\mu_{R} \approx 2 B$.

Let us finally discuss in which range of parameters the non-equilibrium FRG furnishes reliable results. In Fig. 5.8 we show the transport parameters plotted as function of the magnetic field for different bias voltages in the weak $\left(\frac{U}{\pi \Gamma}<1\right.$, Fig. 5.8 (a) ) and intermediate coupling regime $\left(\frac{U}{\pi \Gamma}>1\right.$, Fig. 5.8 (b) ). While for $\frac{U}{\pi \Gamma}<1$ we did not find any parameter regime with a breakdown of the FRG, we see that for $\frac{U}{\pi \Gamma}>1$ the curves acquire a discontinuity in $G$ (see Fig. 5.8 (b) curve with $V_{B} / \Gamma=1$ ). Increasing $V_{B}$ the discontinuity disappears but (for $V_{B} / \Gamma=2 . .3$ ) the conductance overshoots the unitary limit in the range $1<B<2$. This means that our approach is not reliable in this parameter range and possibly for larger magnetic fields. It would be interesting to see whether an energy-dipendent selfenergy and vertex function can extend the range of applicability of the non-equilibrium FRG. 




Figure 5.8: Total conductance normalized to $G_{0}=2 e^{2} / h$ plotted versus $B / \Gamma$ for $V_{G} / \Gamma=0$, and several values of $V_{B}$ in Weak $(U / \Gamma=1,(\mathrm{~b}))$ and strong coupling regime $(U / \Gamma=5$, (a)) 
At higher bias $V_{B} \geq U$ the plot does not show this problem any longer. 


\section{Temperature Dependence of the Transport Parameters}

\subsection{Introduction}

The aim of this chapter is the study of the influence of the temperature $T$ on the transport properties of a single-level quantum dot under a constant applied bias $V_{B}$. First, we will again consider, as limiting case, the equilibrium situation $V_{B} / \Gamma=0$. We will compare our results regarding the conductance $G$ as function of the gate voltage $V_{G}$, for several values of $T$, to the ones coming from the numerical renormalization group (NRG) [88]. Then we will see how $T$ affects the selfenergies resulting from the numerical integration of the system Eq. (4.29)-(4.32). Furthermore, we will show how the current $J$ and the differential conductance $G$ are affected by the temperature and we will see that the transport parameters, calculated by means of the non-equilibrium FRG, reproduce very well the ones obtained by Hershfield et al. [33] with perturbation theory up to second order in the Coulomb repulsion $U$. At the end of this chapter we will show that the non-equilibrium FRG can be used not only when a finite temperature and the magnetic field $B$ are applied separately, but, as long as the Coulomb interaction is not too large, $B$ and $T$ can be "switched on" together. In particular we will show how the interplay of these two scales affects the transport through a single-level quantum dot.

\subsection{Temperature Dependence of the Selfenergies}

In this section we discuss how the energy-independent selfenergies, at the end of the flow $\Lambda=0$, are affected by the temperature $T$, for the electron-hole symmetric case $V_{G} / \Gamma=0$ and in a sligthly asymmetric situation where $V_{G} / \Gamma=0.1$. The essential structure of the flow equations Eq. (5.1)-(4.30) is not modified by the introduction of a finite temperature $T \neq 0$. We note in particular that the $T$-dependence appears in the system of differential equations only through the Fermi functions in the four free dot Green functions Eq. (4.18)-(4.21).

As usual we begin our analysis with the equilibrium case $V_{B} / \Gamma=0$ (see Fig. 6.1 (b)). What we see is that the real part of $\Sigma^{\alpha, \alpha, \Lambda=0}$ remains constant in $T$ and equal to zero for the electron-hole symmetric case. As soon as we move a little bit away from $V_{G} / \Gamma=0, \Re e\left[\sum^{\alpha, \alpha, \Lambda=0}\right]$ is no longer constant, it increases and saturates at 
larger temperatures. The imaginary part of $\Sigma^{\alpha, \alpha, \Lambda=0}$ (for $V_{G} / \Gamma=0.1$ ) is equal to zero in the range $T / \Gamma \approx 0 \ldots 1$ and then it moves slightly away from zero like in the non-equilibrium case at $T=0$.
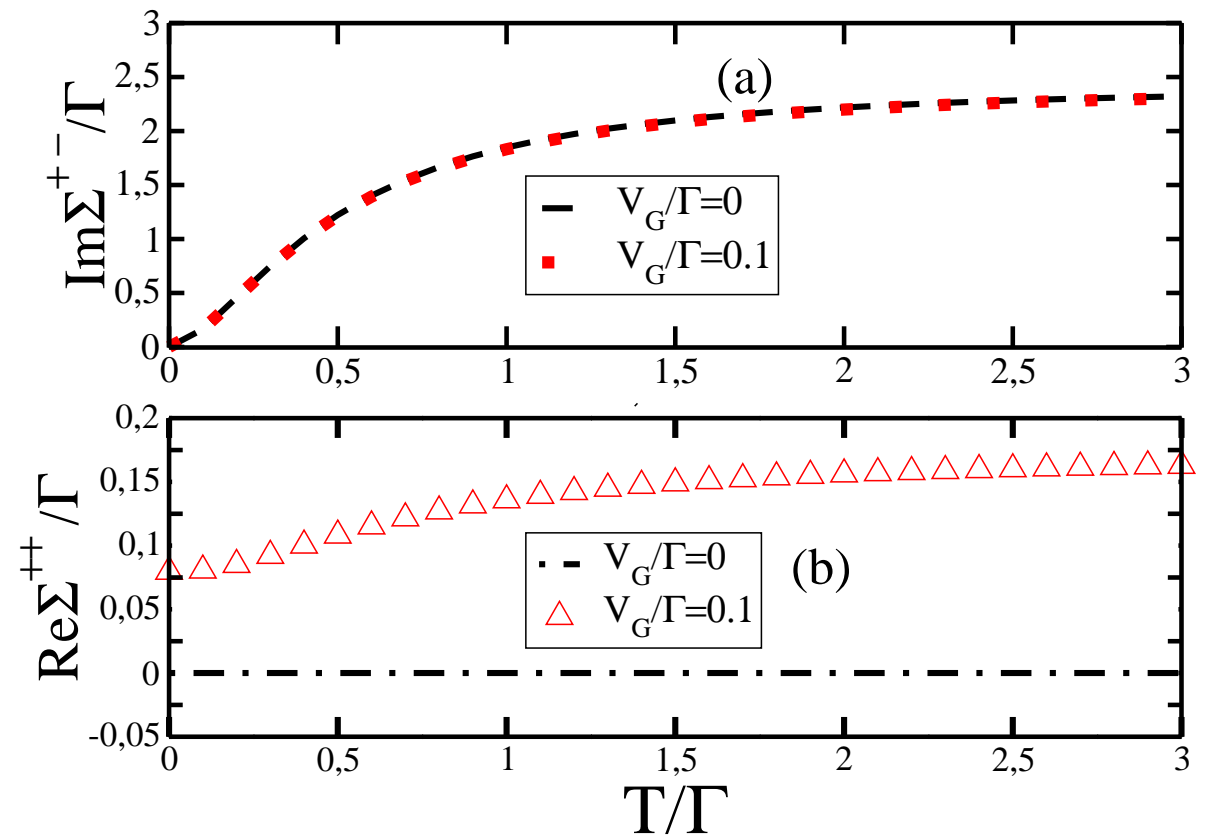

Figure 6.1: (a) Imaginary part $\Sigma^{+-, \Lambda} / \Gamma$, plotted versus $T / \Gamma$ (dashed line $V_{G} / \Gamma=0$, dots $V_{G} / \Gamma=0.1$ ) for $U / \Gamma=5, V_{B} / \Gamma=0$. (b) Flow of real part $\Sigma^{++, \Lambda} / \Gamma$ in the same range of parameter of (a).

Interesting is also the behaviour of the imaginary part of $\Sigma^{+-, \Lambda=0}$ (see Fig. 6.1 (a)). $\Im m\left[\Sigma^{+-, \Lambda=0}\right]$ grows with some power law, for small $T$, then it raises quickly for intermediate $T$ and finally saturates at larger temperatures. To summarize, we can say that, in equilibrium, the temperature acts on the selfenergies in the same qualitative way as the bias voltage $V_{B}$ did in the case $T=0$. Switching on the external voltage $V_{B}$ we observe a difference in the $\Im m\left[\Sigma^{+-, \Lambda=0}\right]$ (see Fig. $6.2(\mathrm{~b})$ ) with respect to the equilibrium case. Now $\Im m\left[\Sigma^{+-, \Lambda=0}\right]$ does not start from zero, at $T / \Gamma=0$, because $V_{B}$ generates off-diagonal components (at $T / \Gamma=0$ ) which renormalize the tunneling rate in the expression of the current and of the life-time broadening (see Eq. 4.50-4.51) The gate voltage $V_{G}$, at least in the small range $0<V_{G} / \Gamma<0.1$, does not affect the temperature behaviour of $\Im m\left[\Sigma^{+-, \Lambda=0}\right]$. The $T$-dependence of $\Re e\left[\Sigma^{\alpha \alpha, \Lambda=0}\right]$ (see Fig. $6.2(\mathrm{a})$ ) is not essentially modified by the introduction of $V_{B}$ with respect to the equilibrium situation.

It is interesting to observe that $T$ has a stronger influence on $\Sigma^{+-, \Lambda=0}$ than on $\Sigma^{\alpha \alpha, \Lambda=0}$. The imaginary part of $\Sigma^{+-, \Lambda=0}$ is enhanced by roughly a factor three in the range $0<T / \Gamma \approx 1$, while the real part of $\Sigma^{\alpha \alpha, \Lambda=0}$ grows slowly and its values 



Figure 6.2: The same parameters range of Fig. 6.1 except for $V_{B} / \Gamma=1$.

remain rather small. This reflects itself in the expression for current Eq. (4.33), since in the integrand of Eq. (4.33) the contributions coming from $\Im m\left[\Sigma^{+-, \Lambda=0}\right]$ are much more relevant then the ones due to $\Sigma^{\alpha \alpha, \Lambda=0}$.

\subsection{Results}

\subsubsection{Equilibrium}

This section is dedicated to the discussion of the temperature behaviour of the conductance $G$ as function of the gate voltage $V_{G}$ in the limit $V_{B} / \Gamma=0$.

We compare the results coming from the non-equilibrium FRG with the ones obtained by the numerical renormalization group (NGR)[88] (see Fig. 6.3 and Fig. 6.5 ). In contrast to the previous chapters, where we compared the non-equilibrium FRG (at $V_{B} / \Gamma=0$ ) to the imaginary-time $\mathrm{FRG}$, we have to take into account another method (NRG) to compare our results in equilibrium, since the imaginarytime FRG does not furnish reliable results at finite temperatures. This could be due to the fact that, within non-equilibrium FRG, no analytical prolongation is needed, while this is a problematic step for the imaginary-time FRG.

The good quality of the results, if $T$ is not too high, is mainly due to two factors: First, the Coulomb interaction is not too large $(U / \Gamma=5)$, therefore the non-equilibrium FRG can reach the same qualitative results given by perturbation 


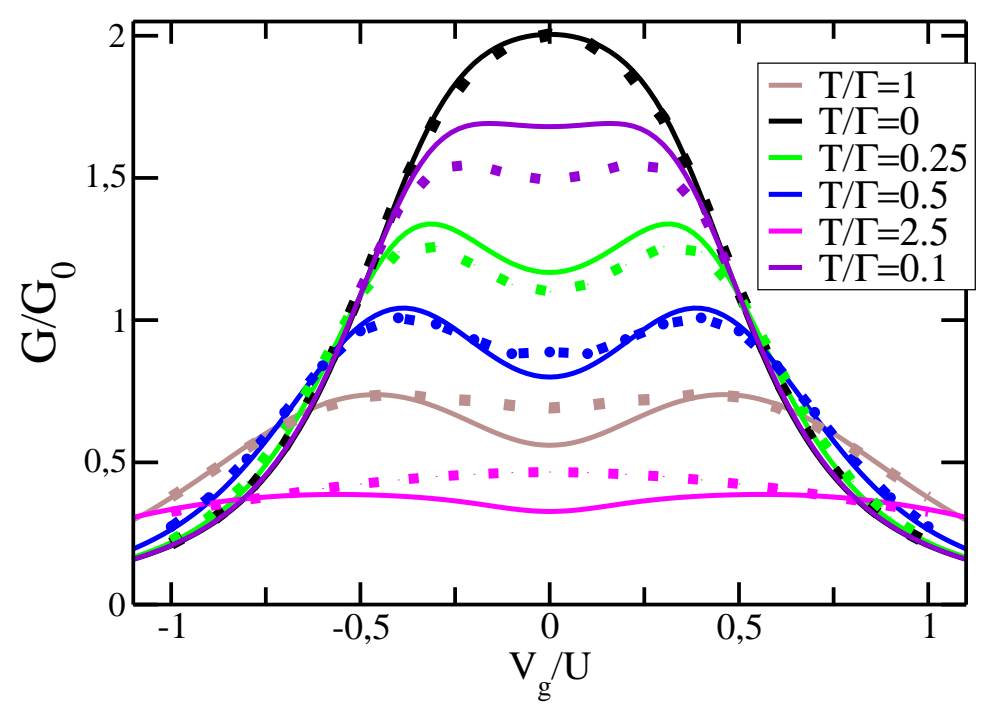

Figure 6.3: Total conductance $G=G_{\uparrow}+G_{\downarrow}$ (normalized to $G_{0}=2 \frac{e^{2}}{h}$ ) as function of the gate voltage $V_{G}$, for $U / \Gamma=5, V_{B} / \Gamma=0, B / \Gamma=0$ and for several temperature $T$. The dot curves represent the results coming from the NRG.

theory. Second, the difference $G^{+-}-G^{-+}$shows a similar behaviour with respect to the spectral density calculated by means of the NRG (see Fig. 6.4 ). In the range between $-0.5<V_{G} / U<0.5$ we note that, at $T \neq 0, G$ is not monotonic. The reason for this is due to the fact that the difference $G^{+-}-G^{-+}$, calculated at $\omega=0$, increases in the range $0<V_{G} / U<0.5$ and then decreases again (see Fig. 6.4), therefore resulting in the non monotonic behaviour depicted in Fig. 6.3 . For larger values of $V_{G} / U$ the difference $G^{+-}-G^{-+}$does not depend any longer on the temperature and we recover again the noninteracting case.

The agreement between the non-equilibrium FRG and the NRG remains good also in an applied magnetic field $B$ as witnessed by Figure 6.5 . In the parameter range in which $B / \Gamma>T / \Gamma$ the conductance peaks, corresponding to the individual spin contributions (spaced by $\approx U / \Gamma$ ), are prominent. As soon as $T / \Gamma \approx B / \Gamma$, the temperature becomes the dominating energy scale and the characteristics peaks induced by the applied magnetic field strongly decrease in magnitude and then disappear, signalling that the correlation between the dot and the leads has disappeared too.

\subsubsection{Current and Conductance as Function of the Applied Voltage}

We begin this section showing the current voltage characteristics, for different temperatures $T$, obtained with the non-equilibrium FRG (see Fig. 6.6). By comparing our results with the ones obtained by Hershfield et al. [33], with perturbation theory 

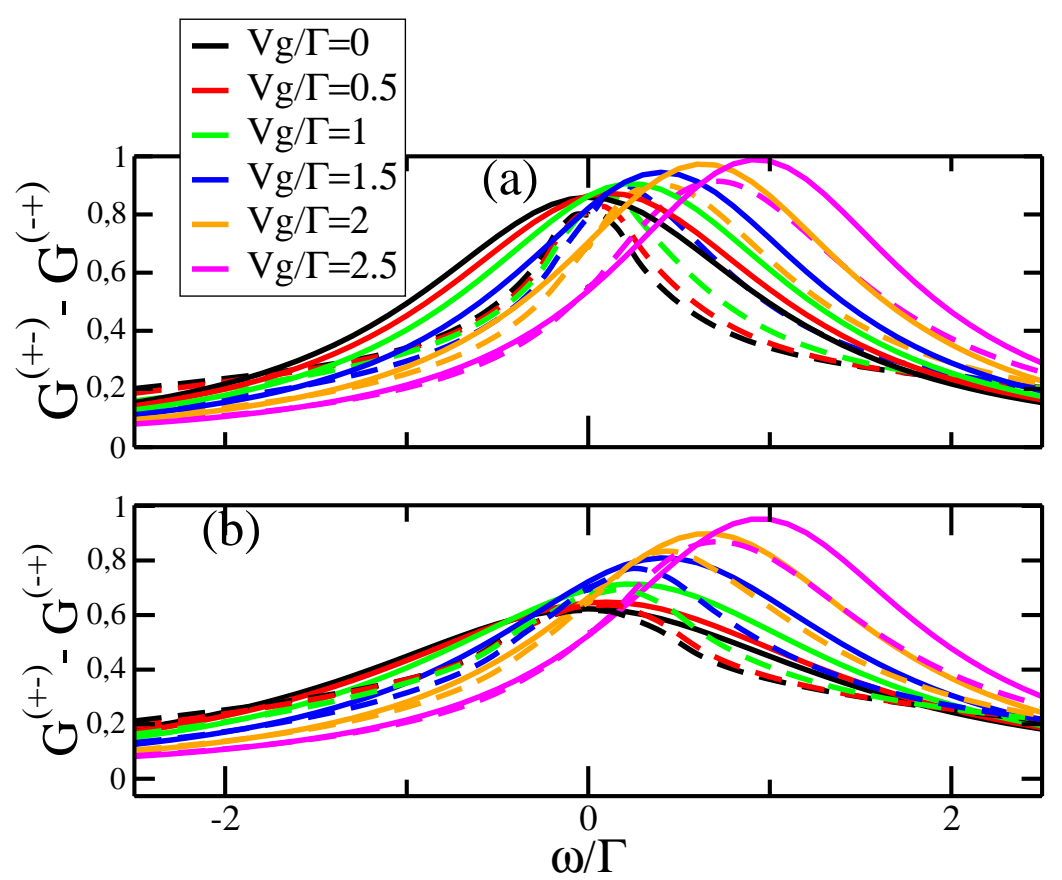

Figure 6.4: Comparison between $G_{d}^{+-}-G_{d}^{-+}$(continuous curves represent results coming from the FRG) and $\rho=-\frac{\Im m\left[G^{R}\right]}{\pi}$ (slashed curves represent the results coming from the NRG ) as function of the frequency $\omega$, for $U / \Gamma=5$ and for several values of the gate voltage $V_{G}$. In the upper plot (a) $T / \Gamma=0.1$, in the lower one $T / \Gamma=0.25$. 




Figure 6.5: The same parameter range of Fig. 6.3 except for $B / \Gamma=0.58$.

up to second order in the Coulomb repulsion $U$, we observe that the non-equilibrium FRG is capable to reproduce the qualitative behaviour of the current calculated using perturbation theory [33]. What we see is that the sharp current increase at $T / \Gamma=0$, for small voltages, which is followed by a shoulder and then by a linear behaviour, is smeared out by the temperature (dotted curve in Fig. 6.6) and finally it is completely suppressed at larger $T$ (dashed curve) in favour of a almost linear current-voltage characteristics. This is due to the fact that $T$ tends to destroy the correlation between the dot and the leads, represented by the spectral function [37]. As direct consequence a decrease in $J$ follows together with the disappeareance of all manybody effects in the current.

In the plot (see Fig. 6.7) we show that within our approach several parameters such as $B, V_{B}$ and $T$ can coexist as long as the Coulomb interaction $U$ is not too large. Roughly speaking the higher $U$ the lower is the magnetic field applicable and the lower is the temperature range in which the non-equilibrium FRG furnishes reliable results. In Figure 6.7 (a)-(b) the current is plotted versus the applied voltage in an external magnetic field, for several temperatures. We see that $B$ tends to shift the current peak to larger $V_{B}$, but even in a parameter range where $B>T$ (see curve at $T / \Gamma=0.1$ ), we observe that a small temperature is enough to smear out the current peak. Furthermore, if we increase $B$ up to $B / \Gamma=0.8$, then we have, for low temperatures (see curve at $T / \Gamma=0.05$ ), a small discontinuity signalling that we are outside of the range of applicability of the non-equilibrium FRG. In any case, as 


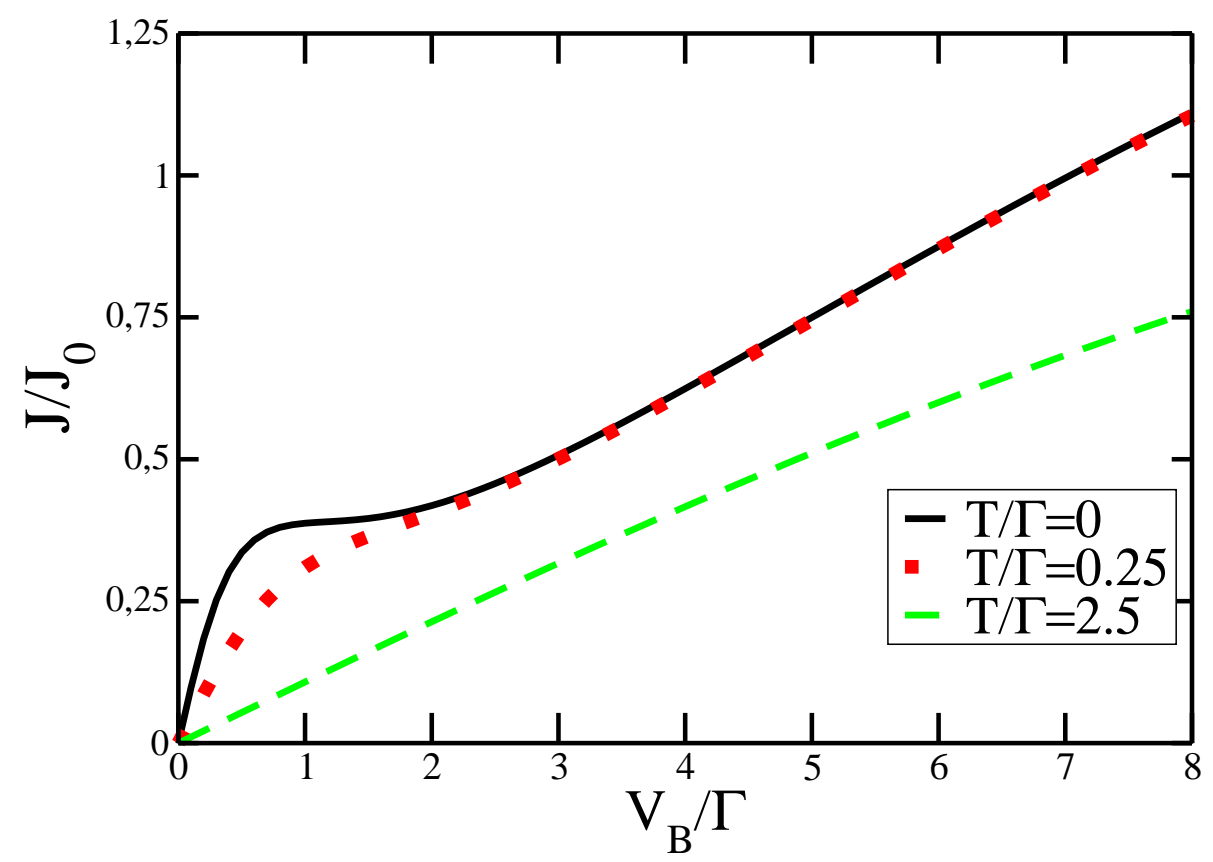

Figure 6.6: Current (normalized to $J_{0}=G_{0} \frac{\Gamma}{e}$ ) as function of $V_{B} / \Gamma$ for $V_{G} / \Gamma=0$, $U / \Gamma=7.51$, and several values of $T$.

soon as $T$ is raised the discontinuity disappears together with the peak, this means that the temperature starts to be the dominating energetic scale.

\subsubsection{Conductance as Function of $B$}

In the following we consider the behaviour of the conductance $G$ as function of the magnetic field, in and off-equilibrium, for several values of $T$. Starting from the equilibrium situation, we see that, for low temperatures (continuous curve in Fig. 6.8 (a)), we still have a relativly sharp peaked $G$. As $B$ grows $G$ decreases and at $B / \Gamma \approx 0.8$ appears a discontinuity and a small region of negative conductance, which again indicates the failure of our method. Enhancing the temperature, but remaining in the range in which $0<B / \Gamma \approx 0.8$, the conductance peak is replaced by a region with constant $G$ up to $B / \Gamma \approx 0.8$ and then $G$ decreases quickly, without showing any kind of discontinuity.

The reason for such a behaviour may be found in the interplay between $B$ and $T$ which takes place in the range $0<T / \Gamma \approx 0.75$. In this range the temperature is not strong enough to suppress the central peak of the spectral density completely. The latter is lowered and broadened indicating a lowering of the correlation between the dot and the electrodes [37]. This means that, as soon as $B$ is large enough to split the spectral density into two subpeaks (at $B / \Gamma \approx 1$ ), the spectral weight is shifted 

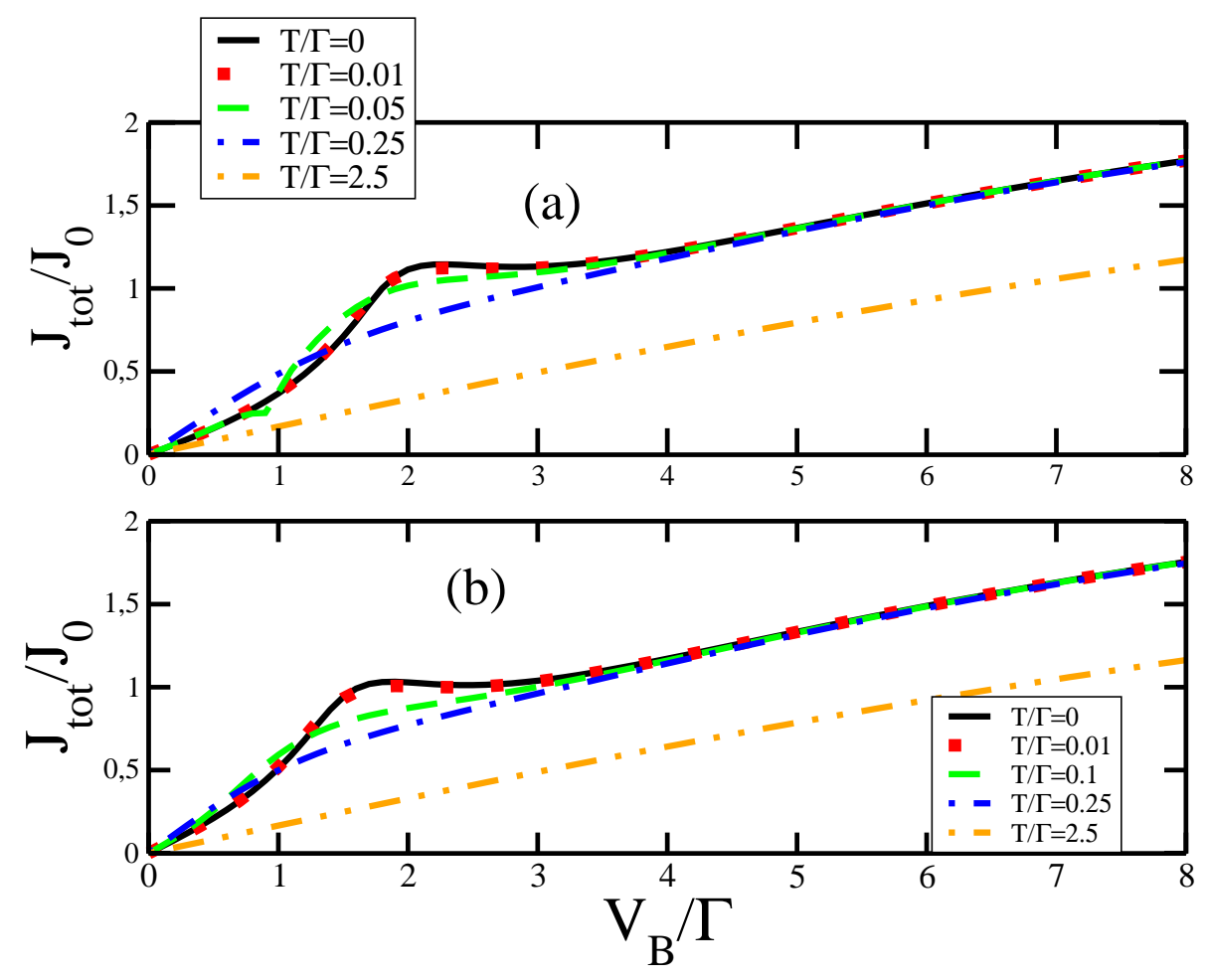

Figure 6.7: Total current: $J_{t o t}=J_{\uparrow}+J_{\downarrow}$ (normalized to $J_{0}=2 G_{0} \frac{\Gamma}{e}$ ) as function of the bias voltage for $V_{G} / \Gamma=0, U / \Gamma=5, B / \Gamma=0.8$ (a), $B / \Gamma=0.6$ (b) and for several temperatures. 
far from the Fermi level and $G$ collapses.

At larger temperatures (above $T / \Gamma=0.75$ ), the spectral weight is far from the Fermi level because the resonance representing the correlation between the dot and the leads does not exist any longer [37]. What remains is just a constant conductance signalling that $T$ has washed out the central resonance in the spectral density. Switching on the bias voltage (see Fig. 6.8 (b)), we observe a discontinuity for small

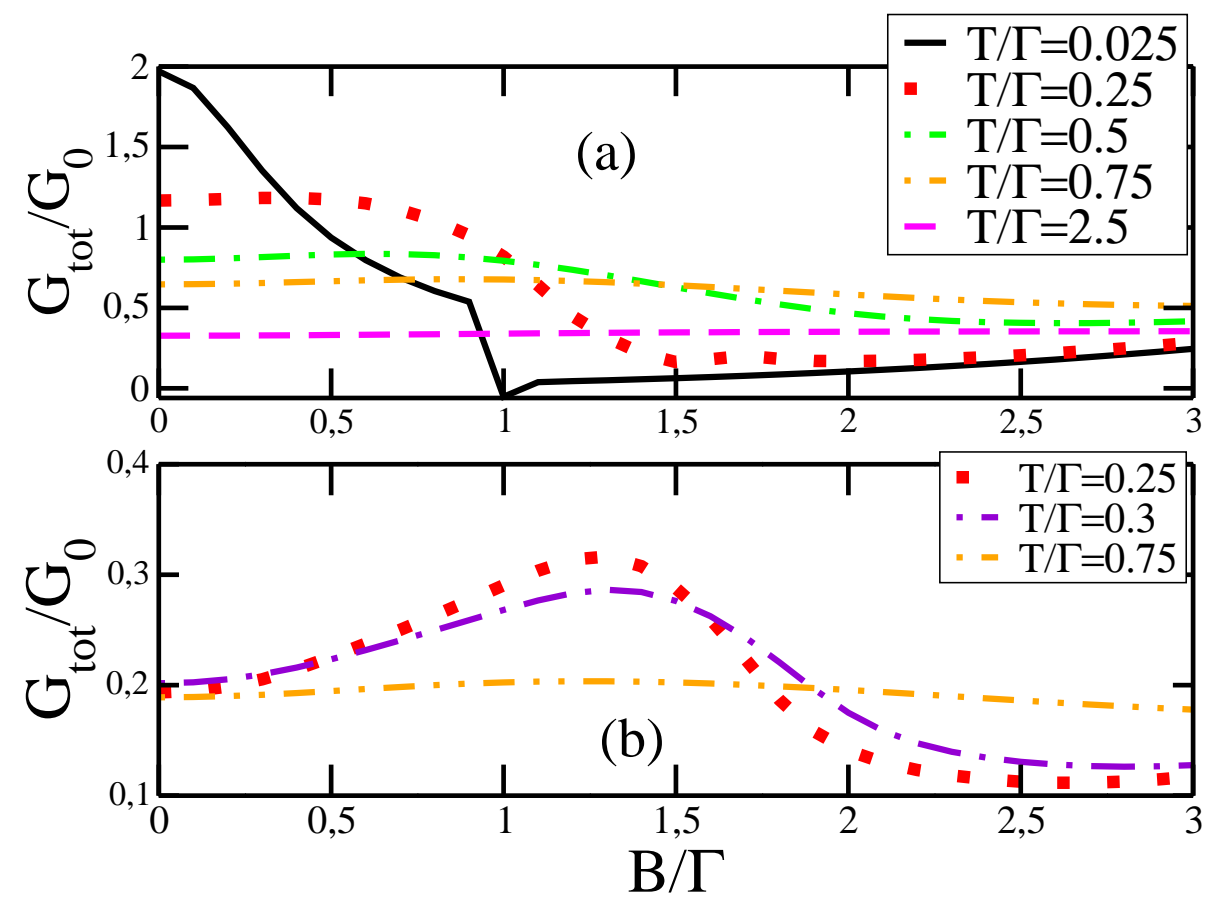

Figure 6.8: Total conductance: $G_{t o t}=G_{\uparrow}+G_{\downarrow}$ (normalized to $G_{0}=2 \frac{\Gamma}{e}$ ) as function of the magnetic field for $V_{G} / \Gamma=0, U / \Gamma=5, V_{B} / \Gamma=0$ (a), $V_{B} / \Gamma=1$ (b) and for several temperatures.

temperatures in the range $0<T / \Gamma \approx 0.1$, which makes the result and therefore the method meaningless. As soon as we consider higher $T$ the problem disappears and we see that the conductance peak is shifted to larger values of $B$, due to the interplay between $V_{B}$ and $B$ (see Section 5.3.3). In any case the peak is again, as expected, not stable versus $T$. In fact we have now another parameter $\left(V_{B}\right)$ which tends to suppress the central resonance in the spectral density, therefore the temperature influence together with $B$ and $T$ destroys the peak in $G$. 


\section{Summary and Outlook}

This work was motivated by the question whether the FRG formalism can be extended to describe the non-equilibrium properties of an interacting quantum manybody system. We were able to derive a formalism that can be used to set up a system of differential equations for e.g. irreducible vertex functions

Although the derivation of the non-equilibrium FRG flow equations is formally identical to the imaginary-time one, the final equations show a more complicated tensor structure. This additional tensor structure is due to the necessity to introduce additional indices taking care of time ordering or, more formally, the different branches of the Keldysh contour. We saw that this formalism is sufficiently general to treat both fermions and bosons at $T \geq 0$. Moreover, the derivation of the non-equilibrium FRG flow equations does not depend on the assumption of a stationary state of the system in question. This means that it can be adopted to describe time-dependent phenomena, too. A further advantage, with respect to the imaginary-time FRG, is due to the fact that the non-equilibrium FRG relies on real quantities. This means that the non-equilibrium FRG does not need any analytical prolongation to the real axis which can be a problematic step in certain cases.

Since the FRG leads to an infinite hierarchy of coupled differential equations, one has to introduce approximations, at least a truncation at a certain level. Typically one neglects the flow of the three-particle vertex. As it has been demonstrated in Ref. [30] for the imaginary-time FRG, one can solve the remaining system of flow equations for simple models like the single impurity Anderson model (SIAM) numerically, keeping the full energy-dependence. Due to the fact that the vertex function carries three continuous frequency arguments in addition to the discrete quantum numbers of the system, such a calculation is computationally quite expensive. To reduce the numerical effort, further approximations can be introduced. A particularly important and successful one is obtained by neglecting the energy dependence of the vertex functions [2], which already leads to a surprisingly accurate description of local and transport properties of interacting quantum dots in the linear response regime.

As a simple but nontrivial application to test our non-equilibrium FRG, we chose the single impurity Anderson model (SIAM). The reason for this choice is that the SIAM represents the paradigm for correlation effects in condensed matter physics. Besides, it is the standard model for the description of the transport properties of interacting single-level quantum dots to which this work has been dedicated.

First, we analyzed the "easiest" case, namely we applied the non-equilibrium FRG 
formalism to the SIAM with finite bias voltage in the stationary state at $T=0$. It turned out, that for the simplest approximation where only the flow of the selfenergy is kept, the analytic structure of the differential equation leads to problems in the numerical solution. In addition, this approximation leads to a violation of the causality relation (Eq. 4.45) to order $U^{2}$. The first problem was resolved by including the two-particle vertex in the flow at least up to the largest interaction considered here $(U / \Gamma=15)$. At the present stage this was for computational reasons done by assuming it to be energy-independent, yielding again an energy-independent selfenergy. Although this approximation also violates Eq. (4.45) to order $U^{2}$ for a fixed $V_{G}$ and $V_{B}$ the error is significantly smaller compared to the simplest scheme. We were able to obtain reasonable expressions and numerical results for the current and the conductance using the functions $G^{\alpha \beta}(\omega)$ instead of $G^{R}(\omega)$ in the current formula. We reproduced nonequilibrium features of the current and differential conductance known from the application of other approximate methods to the SIAM.

In the more advanced truncation scheme and for $V_{G} \neq 0$, the current conservation $\Delta J=0$ only holds to leading order in $U$. This defect can be traced back to the energy independence of the two-particle vertex, leading to finite, but energy-independent $\Sigma^{-+}$and $\Sigma^{+-}$. Unfortunately this deficiency cannot be cured by assuming a coarsegrained energy dependence of the form

$$
F(\omega)= \begin{cases}2, & \text { if } \omega<V_{B} / 2 \\ 1, & \text { if }-V_{B} / 2<\omega<V_{B} / 2 \\ 0, & \text { if } V_{B} / 2<\omega .\end{cases}
$$

since such a minimal energetic lattice is too raw to be able to capture the behaviour of the selfenergy, leading therefore to unphysical results.

Furthermore, we applied an external magnetic field $B$ in order to observe the effect of $B$ on the transport properties of a single-level quantum dot. We saw that, even within our crude approximation, the non-equilibrium FRG furnished reasonable results concerning the transport parameters $J$ and $G$, as long as Coulomb energy and magnetic field are not too large. Besides, we introduced a further approximation, namely we neglected the spin-dependence in the vertex function $\gamma_{2}$ (but not for the selfenergy) and we compared the results with the spin-dependent case, showing and explaining in which range of parameters this second approximation can be adopted. A particularly interesting observation is the switching behaviour in the current, which we could explain with the interplay of the different structures we got in the spectra as function of the gate voltage and $B$. We also showed that, as soon as the bias voltage is switched off $\left(V_{B} \rightarrow 0\right)$, we reobtain, as expected, the linear response results [42]. This means that the non-equilibrium FRG can be also applied to treat systems in equilibrium.

Finally, we considered the temperature dependence in the non-equilibrium FRG. As first we analyzed the equilibrium case comparing our results with the ones coming 
from the numerical renormalization group (NRG). In contrast to the imaginary-time FRG, the non-equilibrium FRG furnishes rasonable results, in small and intermediate coupling regime, at finite temperatures. This could be due to the fact that, within non-equilibrium FRG, no analytical prolongation is needed, while this is a problematic step for the imaginary-time FRG. Afterwards, we studied the temperature dependence of the transport parameters with and without an applied magnetic field.

More interesting is the fact that, for intermediate Coulomb repulsion $U / \Gamma \approx 5$, the non-equilibrium FRG can describe the transport properties of a single-level quantum dot when several external parameters such as the bias, the magnetic field and the temperature are switched on. This is one of the most interesting results we have obtained because, up to now, a theory that allows to access intermediate coupling, bias voltage, magnetic field and temperature on a unique footing is missing.

The main problem still to solve is the introduction of the energy dependence in the non-equilibrium FRG flow equations, in order to get rid of causality problems and to see whether the energy dependence can extend the range of applicability of our method to larger $U$ and to larger $B$ and $T$. 


\section{Appendices}

\subsection{The Morris Lemma}

In this appendix we want to show how to handle products involving a Dirac delta function multiplied by a function whose argument contains a Heaviside step function [64]:

$$
\delta(x) f(\Theta(x)) .
$$

First of all introduce two "smeared" functions

$$
f\left(\Theta_{\varepsilon}(x)\right), \delta_{\varepsilon}(x)
$$

where $f\left(\Theta_{\varepsilon}\right)(x)$ is an any function which remains continuous in the limit $\varepsilon \rightarrow 0$ and

$$
\delta_{\varepsilon}(x):=-\frac{d}{d x} \Theta_{\varepsilon}(x) \rightarrow \delta(x)
$$

as $\varepsilon \rightarrow 0$. Out of the last two equations and from the Newton-Liebnitz theorem we can write Eq.(8.1) as

$$
\begin{aligned}
\delta(x) f(\Theta(x)) & =\lim _{\varepsilon \rightarrow 0} \delta_{\varepsilon}(x) f\left(\Theta_{\varepsilon}(x)\right)=\left.\lim _{\varepsilon \rightarrow 0} \frac{d}{d x} \int_{0}^{\Theta_{\varepsilon}(x)} d t f(t)\right|_{x^{\prime}=x}= \\
= & \frac{d}{d x}\left[\theta(x) \int_{0}^{1} f(t) d t\right]=\delta(x) \int_{0}^{1} d t f(t),
\end{aligned}
$$

In order to obtain the Eq. (8.3), we have supposed that it is possible to exchange the limit with the derivative, moreover we have used the following relation:

$$
\frac{d}{d x}\left[\lim _{\varepsilon \rightarrow 0} \int_{0}^{\Theta_{\varepsilon}(x)} f(t) d t\right]=\left\{\begin{array}{rll}
\frac{d}{d x} \int_{0}^{1} f(t) d t & \text { for } & x>0 \\
0 & \text { for } & x<0
\end{array}\right.
$$

The r.h.s. of Eq. (8.3) is what we need to handle (as we will see in the next paragraph) equations involving the single scale propagator $\mathcal{S}^{\Lambda}$ and products between it and the Green functions $G^{\Lambda}$. 


\subsection{Expressions Involving the Single Scale Propagator}

In order to be able to treat and simplify products involving $\mathcal{S}^{\Lambda} G^{\Lambda}$ we will apply basically what we have learnt in the previous section regarding the Morris lemma. We begin with the easiest case, namely the single scale propagator, then we will take into account the product $\mathcal{S}^{\Lambda} G^{\Lambda}$. In the following we won't use the hat to indicate the matrices, but keep in mind that $\mathcal{S}^{\Lambda}$ and $G^{\Lambda}$ must be rad as matrices with respect to the Keldysh indices. We start with the definition of the cutoff-Green functions and single scale propagator

$$
\begin{gathered}
G_{0}^{\Lambda}(\omega)=\Theta(|\omega|-\Lambda) G_{0}^{\Lambda}(\omega), \\
\left.G_{(}^{\Lambda} \omega\right)=\frac{1}{\left(G_{0}^{\Lambda}(\omega)\right)^{-1}-\Sigma^{\Lambda}(\omega)}, \\
\mathcal{S}^{\Lambda}(\omega)=G^{\Lambda}(\omega) \frac{d}{d \Lambda}\left[\left(G_{0}^{\Lambda}(\omega)\right)^{-1}\right] G^{\Lambda}(\omega) .
\end{gathered}
$$

Inserting the Eq. (8.4) into Eq. (8.5)

$$
\begin{gathered}
\mathcal{S}^{\Lambda}(\omega)=G^{\Lambda}(\omega) \frac{d}{d \Lambda}\left[G_{0}(\omega)\right]^{-1}\left[(\Theta(|\omega|-\Lambda))^{-1}\right] G^{\Lambda}(\omega)= \\
G^{\Lambda}(\omega)\left[G_{0}(\omega)\right]^{-1}[-\delta(|\omega|-\Lambda)]\left[(\Theta(|\omega|-\Lambda))^{-2}\right] G^{\Lambda}(\omega)=
\end{gathered}
$$

Multiplying in the matrix sense $G_{0} * G_{0}^{-1}=\mathbf{1}$ to the right results in

$$
\begin{gathered}
G^{\Lambda}(\omega)\left[G_{0}^{-1} \Theta(|\omega|-\Lambda)\right]\left[-\delta(|\omega|-\Lambda) G_{0}(\omega)\right]\left[G_{0}^{-1} \Theta(|\omega|-\Lambda)\right] G^{\Lambda}(\omega)= \\
{\left[G^{\Lambda}(\omega) G_{0}^{\Lambda,-1}\right]\left[-\delta(|\omega|-\Lambda) G_{0}(\omega)\right]\left[G_{0}^{\Lambda,-1}(\omega) G^{\Lambda}(\omega)\right]}
\end{gathered}
$$

where we have used Eq. (8.4) in the last step. Applying the Dyson equation morover delivers

$$
\mathcal{S}^{\Lambda}(\omega)=\left[\frac{1}{1-G_{0}^{\Lambda}(\omega) \Sigma^{\Lambda}(\omega)}\right]\left[-\delta(|\omega|-\Lambda) G_{0}(\omega)\right]\left[\frac{1}{1-\Sigma^{\Lambda}(\omega) G_{0}^{\Lambda}(\omega)}\right]
$$

Substituting the right square bracket, by means of the Dyson equation using

$\frac{1}{1-G_{0}^{\Lambda} \Sigma^{\Lambda}}=G_{0}^{-1} \frac{1}{1-\Sigma^{\Lambda} G_{0}^{\Lambda}}$ and applying the matrix identity $[A B]^{-1}=B^{-1} A^{-1}$ to the previous expression we obtain

$$
\mathcal{S}^{\Lambda}(\omega)=\frac{1}{\left[1-\Sigma^{\Lambda(\omega)} G_{0}^{\Lambda}(\omega)\right]^{2}} G_{0}(\omega) \delta(|\omega|-\Lambda) .
$$


Eq. (8.6) must now be rewritten through the Morris lemma

$$
\begin{gathered}
\frac{1}{\left[1-\Sigma^{\Lambda}(\omega) G_{0}^{\Lambda}(\omega)\right]^{2}} G_{0}(\omega) \delta(|\omega|-\Lambda) \rightarrow G_{0} \delta(|\omega|-\Lambda) \int_{0}^{1} d x \frac{1}{\left[1-x \Sigma^{\Lambda}(\omega) G_{0}^{\Lambda}(\omega)\right]^{2}}= \\
=\delta(|\omega|-\Lambda) \frac{1}{\left(G_{0}(\omega)\right)^{-1}-\Sigma^{\Lambda}(\omega)} .
\end{gathered}
$$

Therefore we can write

$$
\mathcal{S}^{\Lambda}(\omega) \rightarrow \delta(|\omega|-\Lambda) \frac{1}{\left(G_{0}\right)^{-1}(\omega)-\Sigma^{\Lambda}(\omega)} .
$$

\subsubsection{More Complicated Products}

We are now interested in simplifications of products involving

$$
\mathcal{S}^{\Lambda}(\omega) G^{\Lambda}\left(\omega^{\prime}\right)
$$

Inserting Eq. (8.7) into Eq. (8.8) we obtain

$$
\mathcal{S}^{\Lambda}(\omega) G^{\Lambda}\left(\omega^{\prime}\right)=\left[\delta(|\omega|-\Lambda) \frac{1}{\left(G_{0}\right)^{-1}(\omega)-\Sigma^{\Lambda}(\omega)}\right] * \frac{1}{\left(G_{0}\left(\omega^{\prime}\right)\right)^{-1}-\Sigma^{\Lambda}\left(\omega^{\prime}\right)}
$$

We then right right-multiply with $G_{0}\left(\omega^{\prime}\right) \Theta\left(\left|\omega^{\prime}\right|-\Lambda\right)$

$$
\begin{aligned}
& {\left[\delta(|\omega|-\Lambda) \frac{1}{\left(G_{0}\right)^{-1}-\Sigma^{\Lambda}(\omega)}\right] * \frac{G_{0}\left(\omega^{\prime}\right) \Theta\left(\left|\omega^{\prime}\right|-\Lambda\right)}{1-\Sigma^{\Lambda}\left(\omega^{\prime}\right) G_{0}^{\Lambda}\left(\omega^{\prime}\right)}=} \\
& {\left[\delta(|\omega|-\Lambda) \frac{1}{\left(G_{0}\right)^{-1}-\Sigma^{\Lambda}(\omega)}\right] * \frac{\Theta\left(\left|\omega^{\prime}\right|-\Lambda\right)}{\left(G_{0}\right)^{-1}\left(\omega^{\prime}\right)-\Sigma^{\Lambda}\left(\omega^{\prime}\right)} .}
\end{aligned}
$$

Out of the latter can we therefore write Eq. (8.8) as

$$
\mathcal{S}^{\Lambda}(\omega) G^{\Lambda}\left(\omega^{\prime}\right) \rightarrow \delta(|\omega|-\Lambda) \Theta\left(\left|\omega^{\prime}\right|-\Lambda\right) G^{\Lambda}(\omega) G^{\Lambda}\left(\omega^{\prime}\right)
$$

\subsection{Derivation of Eq. (3.11)}

The goal of this section is the detailed derivation of Eq. (3.11). To this end start with the logarithm of the generator of the connected Green functions

$$
\operatorname{Ln}\left[\mathcal{W}^{\Lambda}(\{\bar{\eta}\},\{\eta\})\right]:=\mathcal{W}^{c, \Lambda}(\{\bar{\eta}\},\{\eta\})=
$$




$$
\operatorname{Ln}\left[\frac{1}{\Xi_{0}^{\Lambda}} \int \mathcal{D} \bar{\psi} \psi \exp \left\{\left(\bar{\psi},\left[\hat{G}_{0}^{\Lambda}\right]^{-1} \psi\right)-i S_{\mathrm{int}}(\{\bar{\psi}\},\{\psi\})-(\bar{\psi}, \eta)-(\bar{\eta}, \psi)\right\}\right]
$$

with

$$
\Xi_{0}^{\Lambda}=\int \mathcal{D} \bar{\psi} \psi \exp \left\{\left(\bar{\psi},\left[\hat{G}_{0}^{\Lambda}\right]^{-1} \psi\right)\right\}
$$

Differentatiating $\mathcal{W}^{c, \Lambda}$ with respect to $\Lambda$ results in

$$
\begin{aligned}
\frac{d \mathcal{W}^{c, \Lambda}}{d \Lambda}= & \frac{1}{\mathcal{W}^{\Lambda}} \int \mathcal{D} \bar{\psi} \psi \frac{d}{d \Lambda}\left[\frac{1}{\Xi_{0}^{\Lambda}} \exp \left\{\left(\bar{\psi},\left[\hat{G}_{0}^{\Lambda}\right]^{-1} \psi\right)\right\}\right] \\
& \times \exp \left\{-i S_{\mathrm{int}}-(\bar{\psi}, \eta)-(\bar{\eta}, \psi)\right\} \\
= & \dot{\mathcal{W}}^{c, \Lambda}
\end{aligned}
$$

We now have to evaluate the derivative appearing in the integrand in Eq. (8.11) obtaining

$$
\begin{aligned}
& \frac{d}{d \Lambda} \frac{1}{\Xi_{0}^{\Lambda}} \exp \left\{\left(\bar{\psi},\left[\hat{G}_{0}^{\Lambda}\right]^{-1} \psi\right)\right\}=\frac{1}{\Xi_{0}^{\Lambda}}\left(\bar{\psi},\left[\mathcal{G}^{0, \Lambda}\right]^{-1} \psi\right) \exp \left\{\left(\bar{\psi},\left[\hat{G}_{0}^{\Lambda}\right]^{-1} \psi\right)\right\}- \\
& -\left(\frac{\dot{\Xi}_{0}^{\Lambda}}{\left(\Xi_{0}^{\Lambda}\right)^{2}}\right) \exp \left\{\left(\bar{\psi},\left[\hat{G}_{0}^{\Lambda}\right]^{-1} \psi\right)\right\},
\end{aligned}
$$

leading us by insertion into Eq. (8.11)

$$
\begin{aligned}
& \frac{d \mathcal{W}^{c, \Lambda}}{d \Lambda}=\frac{1}{\mathcal{W}^{\Lambda}} \frac{-\dot{\Xi}_{0}^{\Lambda}}{\left(\Xi_{0}^{\Lambda}\right)^{2}} \int \mathcal{D} \bar{\psi} \psi \exp \left\{\left(\bar{\psi},\left[\hat{G}_{0}^{\Lambda}\right]^{-1} \psi\right)\right\} F(\{\bar{\eta}\},\{\eta\})+ \\
& +\frac{1}{\mathcal{W}^{\Lambda}} \frac{1}{\Xi_{0}^{\Lambda}} \int \mathcal{D} \bar{\psi} \psi\left(\bar{\psi},\left[\hat{\dot{G}}_{0}^{\Lambda}\right]^{-1} \psi\right) \exp \left\{\left(\bar{\psi},\left[\hat{G}_{0}^{\Lambda}\right]^{-1} \psi\right)\right\} F(\{\bar{\eta}\},\{\eta\}),
\end{aligned}
$$

where $F(\{\bar{\eta}\},\{\eta\}):=\exp \left\{-i S_{\text {int }}(\{\bar{\psi}\},\{\psi\})-(\bar{\psi}, \eta)-(\bar{\eta}, \psi)\right\}$. Explicitely writing

$$
\dot{\Xi}_{0}^{\Lambda}=\int \mathcal{D} \bar{\psi} \psi\left(\bar{\psi},\left[\hat{\dot{G}}_{0}^{\Lambda}\right]^{-1} \psi\right) \exp \left\{\left(\bar{\psi},\left[\hat{G}_{0}^{\Lambda}\right]^{-1} \psi\right)\right\}
$$


by means of the substitution into Eq. (8.12) we obtain ${ }^{1}$

$$
\begin{aligned}
& \frac{d \mathcal{W}^{c, \Lambda}}{d \Lambda}=\frac{1}{\mathcal{W}^{\Lambda}} \frac{-\mathcal{W}^{\Lambda}}{\Xi_{0}^{\Lambda}} \int \mathcal{D} \bar{\psi} \psi\left(\bar{\psi},\left[\hat{\dot{G}}_{0}^{\Lambda}\right]^{-1} \psi\right) \exp \left\{\left(\bar{\psi},\left[\hat{\dot{G}}_{0}^{\Lambda}\right]^{-1} \psi\right)\right\}+ \\
& \frac{1}{\mathcal{W}^{\Lambda}} \frac{1}{\Xi_{0}^{\Lambda}} \int \mathcal{D} \bar{\psi} \psi\left(\bar{\psi},\left[\hat{\dot{G}}_{0}^{\Lambda}\right]^{-1} \psi\right) \exp \left\{\left(\bar{\psi},\left[\hat{G}_{0}^{\Lambda}\right]^{-1} \psi\right)\right\} F(\{\bar{\eta}\},\{\eta\}) .
\end{aligned}
$$

Let us look at the first term of the previous equation in terms of a coordinate representation of a scalar product

$$
\left(\bar{\psi},\left[\hat{\dot{G}}_{0}^{\Lambda}\right]^{-1} \psi\right)=i \sum_{m, l} \bar{\psi}_{l} \dot{g}_{l m, 0}^{\Lambda,-1} \psi_{m} .
$$

The factors $\dot{g}_{l m, 0}^{\Lambda,-1}$ do not depend on $\psi$, therefore they can be moved out of the integral

$$
\begin{aligned}
& \frac{d \mathcal{W}^{c, \Lambda}}{d \Lambda}=-(i \zeta) \sum_{m, l} \dot{g}_{l m, 0}^{\Lambda,-1} \int \frac{\mathcal{D} \psi \bar{\psi}}{\Xi_{0}^{\Lambda}}\left(\psi_{l} \bar{\psi}_{m}\right) \exp \left\{\left(\bar{\psi},\left[\hat{\dot{G}}_{0}^{\Lambda}\right]^{-1} \psi\right)\right\} F(\{\bar{\eta}\},\{\eta\})+ \\
& \quad+\frac{1}{\mathcal{W}^{\Lambda}} \int \frac{\mathcal{D} \psi \bar{\psi}}{\Xi_{0}^{\Lambda}}\left(\bar{\psi},\left[\hat{\dot{G}}_{0}^{\Lambda}\right]^{-1} \psi\right) \exp \left\{\left(\bar{\psi},\left[\hat{G}_{0}^{\Lambda}\right]^{-1} \psi\right)\right\} F(\{\bar{\eta}\},\{\eta\}) .
\end{aligned}
$$

The integral in the first term of Eq. (8.14) is exactly the definition of the 1-particle Green function

$$
\left[G_{0}^{\Lambda}\right]_{m l}=i \zeta\left\langle\psi_{l} \bar{\psi}_{m}\right\rangle_{0}
$$

which we reexpress in a trace form

$$
\begin{gathered}
\frac{d \mathcal{W}^{c, \Lambda}}{d \Lambda}=-\operatorname{Tr}\left(\left[\dot{G}^{0, \Lambda}\right]^{-1} G_{0}^{\Lambda}\right)+ \\
\frac{1}{\mathcal{W}^{\Lambda}} \int \frac{\mathcal{D} \psi \bar{\psi}}{\Xi_{0}^{\Lambda}} \bar{\psi} \psi\left(\bar{\psi},\left[\hat{\dot{G}}_{0}^{\Lambda}\right]^{-1} \psi\right) \exp \left\{\left(\bar{\psi},\left[\hat{G}_{0}^{\Lambda}\right]^{-1} \psi\right)\right\} F(\{\bar{\eta}\},\{\eta\}) .
\end{gathered}
$$

Let us take into account the second term of Eq.(8.15) and use again Eq. (8.13) together with

$$
\psi_{l}=i \frac{\delta}{\delta \bar{\eta}_{l}}
$$

\footnotetext{
${ }^{1}$ Here we have used

$$
\mathcal{W}^{\Lambda}=\frac{1}{\left(\Xi_{0}^{\Lambda}\right)} \int \mathcal{D} \bar{\psi} \psi \exp \left\{\left(\bar{\psi},\left[\hat{G}_{0}^{\Lambda}\right]^{-1} \psi\right)\right\} F(\{\bar{\eta}\},\{\eta\}) .
$$
}




$$
\bar{\psi}_{m}=-i \frac{\delta}{\delta \eta_{m}}
$$

Eq. (8.15) then becomes

$$
\begin{gathered}
\frac{d \mathcal{W}^{c, \Lambda}}{d \Lambda}=-\operatorname{Tr}\left(\left[\dot{G}^{0, \Lambda}\right]^{-1} G_{0}^{\Lambda}\right)- \\
-i^{3} \frac{1}{\mathcal{W}^{\Lambda}} \sum_{l, m}\left(\dot{g}_{l m, 0}^{\Lambda,-1} \frac{\delta}{\delta \bar{\psi}_{l}} \frac{\delta}{\delta \psi_{m}}\right)\left(\int \frac{\mathcal{D} \psi \bar{\psi}}{\Xi_{0}^{\Lambda}} \exp \left\{\left(\bar{\eta},\left[\hat{G}_{0}^{\Lambda}\right]^{-1} \eta\right)\right\} F(\{\bar{\eta}\},\{\eta\})\right) .
\end{gathered}
$$

The second term of Eq. (8.16) can be arranged as scalar product

$$
\frac{d \mathcal{W}^{c, \Lambda}}{d \Lambda}=-\operatorname{Tr}\left(\left[\dot{G}^{0, \Lambda}\right]^{-1} G_{0}^{\Lambda}\right)+i \frac{1}{\mathcal{W}^{\Lambda}}\left(\frac{\delta}{\delta \bar{\eta}}, \hat{G}_{0}^{\Lambda,-1} \frac{\delta}{\delta \bar{\eta}}\right) \mathcal{W}^{\Lambda}
$$

which can be furtherly transformed according to

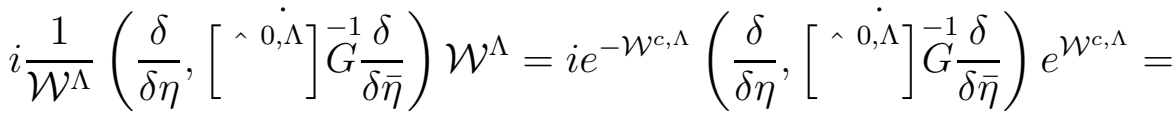



where we used

$$
\mathcal{W}^{c, \Lambda}:=\operatorname{Ln}\left[\mathcal{W}^{\Lambda}\right]
$$

in the first step, from which follows

$$
\mathcal{W}^{\Lambda}=e^{\mathcal{W}^{c, \Lambda}}
$$

By applying the cyclic property of the trace to the first term of Eq. (8.18) we obtain

$$
i \operatorname{Tr}\left(\left[\left[{ }^{\wedge} \cdot \dot{0},\right]^{-1}\right]^{t} \frac{\delta^{2} \mathcal{W}^{c, \Lambda}}{\delta \eta \delta \bar{\eta}}\right)=i \zeta \operatorname{Tr}\left(\frac{\delta^{2} \mathcal{W}^{c, \Lambda}}{\delta \bar{\eta} \delta \eta}\left[{ }^{\wedge} \cdot \dot{0, \Lambda}\right]^{-1}\right),
$$

where the $\zeta$ factor comes from the Grassmann rules. The latter expression, inserted into Eq. (8.18), and then into Eq. (8.17) permits us to finally obtain Eq. (3.11)

$$
\frac{d \mathcal{W}^{c, \Lambda}}{d \Lambda}=-\operatorname{Tr}\left(\left[\dot{G}^{0, \Lambda}\right]^{-1} G_{0}^{\Lambda}\right)+i \zeta \operatorname{Tr}\left(\frac{\delta^{2} \mathcal{W}^{c, \Lambda}}{\delta \bar{\eta} \delta \eta}\left[{ }^{\wedge} \dot{0, \Lambda}\right] \bar{G}\right)+i\left(\frac{\delta \mathcal{W}^{c, \Lambda}}{\delta \eta},\left[{ }^{\wedge} \cdot \dot{0, \Lambda}\right]^{-1} \frac{\delta \mathcal{W}^{c, \Lambda}}{\delta \bar{\eta}}\right)
$$




\section{Bibliography}

[1] B. Altshuler and Y. Aharonov, JETP 48, 812 (1978).

[2] S. Andergassen, T. Enss, and V. Meden, Phys. Rev. B 73, 153308 (2006).

[3] F. B. Anders and A. Schiller (2006), cond-mat/0604517.

[4] P. W. Anderson, Phys. Rev. 124, 41 (1961).

[5] C.W.J. Beenakkerand H. Van Houten, Phys. Rev. Lett. 66, 3056 (1991).

[6] M.T. Bjork, C Thelander, A.E. Hansen, L.E. Jensen, M.W. Larsson, L.R. Wallenberg, L. Samuelson, and Nano Letters 4, 1621 (2004).

[7] L. Canet, B. Delamotte, O. Deloubriere, and N. Wschebor, Phys. Rev. Lett. 92, 195703 (2004).

[8] T. Costi, Phys. Rev. B 55, 3003 (1997).

[9] T. Costi, Phys. Rev. B 64, 241310(R) (2001).

[10] R. A. Craig, J. Math. Phys. 9, 605 (1968).

[11] S.M. Cronenwett and et al. Rep. Prog. Phys. 64, 701 (2001).

[12] P. Danielewicz, Annals of Physics 152, 239 (1984).

[13] S. Datta (2006), cond-mat/0603034.

[14] A. Fetter and J. Walecka, Quantum Theory of Many-Particle Systems, International Series in Pure and Applied Physics (McGraw-Hill, New York, 1971).

[15] G. A. Fiete and E. J. Heller, Rev. Mod. Phys. 75, 933 (2003).

[16] T. Fujii and K. Ueda, Phys. Rev. B 68, 155310 (2003).

[17] T.A. Fulton, G.J. Dolan, Phys. Rev. Lett. 59, 109 (1987).

[18] A. Georges, G. Kotliar, W. Krauth, and M. J. Rozenberg, Rev. Mod. Phys. 68, 13 (1996).

[19] R. Gezzi, T. Pruschke, and V. Meden, Phys. Rev. B 75, 045324 (2007). 
[20] S. D. Glazek and P. B. Wiegmann, Phys. Rev. D 48, 5863 (1993).

[21] L.I. Glazman, M.E. Raikh, JETP Lett. 47, 452 (1988).

[22] D. Goldhaber-Gordon and et al. (1997), Cond-mat/9707311.

[23] C.J. Gorter, Physica 17, 777 (1951).

[24] G. Gruener and A. Zawadowski, Rep.Prog.Phys. 37, 1497 (1974).

[25] M. Hamasaki (2005), cond-mat/0506752.

[26] J. H. Han (2004), cond-mat/0405477.

[27] J. E. Han, Phys. Rev. B 73, 125319 (2006).

[28] J. E. Han (2006), cond-mat/0604583.

[29] H. Haug and A.-P. Jauho, Quantum Kinetics and Optics of Semiconductors (Springer Verlag, 1996).

[30] R. Hedden, V. Meden, T. Pruschke, and K. Schönhammer, J. Phys.: Condens. Matter 16, 5279 (2004).

[31] R. Hedden Single Particle Dynamics of Anderson-like Impurity Models : A Functional Renormalization Group Study,Ph.D. thesis, Goettingen 2007

[32] S. Hershfield, J. H. Davies, and J. W. Wilkins, Phys. Rev. Lett. 67, 3720 (1991).

[33] S. Hershfield, J. H. Davies, and J. W. Wilkins, Phys. Rev. B 46, 7046 (1992).

[34] A. C. Hewson, The Kondo Problem to Heavy Fermions, Cambridge Studies in Magnetism (Cambridge University Press, Cambridge, 1993).

[35] C. Honerkamp, D. Rohe, S. Andergassen, and T. Enss, Phys. Rev. B 70, 235115 (2004).

[36] C. Honerkamp and M. Salmhofer, Phys. Rev. B 67, 174504 (2003).

[37] B. Horvatic D. Sokcevicand and V. Zlatic, Phys. Rev. B 36, 675 (1987).

[38] S. Jakobs, Diploma thesis, RWTH Aachen (2003).

[39] S. Jakobs, V. Meden, and H. Schoeller, in preparation.

[40] L.P. Kadanoff, G. Baym, Quantum Statistical Mechanics: Green's Function Methods in Equilibrium and Nonequilibrium Problems (Addison-Wesley, 1962). 
[41] A. Kamenev, in Les Houches, Volume Session LX, edited by H. Bouchiat, Y. Gefen, S. Guéron, G. Montambaux, and J. Dalibard (Elsevier, NorthHolland, 2004), cond-mat/0412296.

[42] C. Karrasch, T. Enss, and V. Meden, Phys. Rev. B 73, 235337 (2006).

[43] M. A. Kastner, Rev. Mod. Phys. 64, 849 (1992).

[44] M. Keil and H. Schoeller, Phys. Rev. B 63, 180302 (2001).

[45] L. P. Keldysh, JETP 20, 1018 (1965).

[46] A. Komnik and A. O. Gogolin, Phys. Rev. Lett. 94, 216601 (2005).

[47] J. Kondo, Prog. Theor. Phys. 32, 37 (1964).

[48] V. Koremann, Ann. of Phys. 39, 72 (1966).

[49] L. P. Kouwenhoven et al., in Mesoscopic Electron Transport, edited by L. L. Sohn et al. (Dordrecht: Kluwer, 1997), p. 105.

[50] L. P. Kouwenhoven, D. G. Austing, and S. Tarucha, Rep. Prog. Phys. 64, 701 (2001).

[51] L.P. Kouwenhoven D.G. Austing and S. Tarucha (2001), Cond-mat/9707311.

[52] L. Landau and E. Lifshitz, Physical Kinetics (Akademie-Verlag Berlin, 1983).

[53] L. Landau and E. Lifshitz, Theorie des kondensierten Zustandes (AkademieVerlag Berlin, 1980).

[54] D. Langreth, in NATO advanced study institute Series B, edited by J. Devreese and E. van Doren (Plenum New York/London, 1967), vol. 17.

[55] A. Levy Yeyati, A. Martín-Rodero, and F. Flores, Phys. Rev. Lett. 71, 2991 (1992).

[56] J. Li, W.-D. Schneider, R. Berndt, and B. Delley, Phys. Rev. Lett. 80, 2893 (1998).

[57] D. Lobaskin and S. Kehrein, Phys. Rev. B 71, 193303 (2005).

[58] V. Madhavan, W. Chen, T. Jamneala, M. F. Crommie, and N. S. Wingreen, Science 280, 567 (1998).

[59] G.D. Mahan, Many Particle Physics New York:Plenum (1990). 
[60] T. Maier, M. Jarrell, T. Pruschke, and M. H. Hettler, Rev. Mod. Phys. 77, 1027 (2005).

[61] V. Meden, lecture notes on the "Functional renormalization group", http://www.theorie.physik.uni-goettingen.de/ meden/funRG/

[62] Y. Meir, N. S. Wingreen, and P. A. Lee, Phys. Rev. Lett. 70, 2601 (1993).

[63] Y. Meir and N. S. Wingreen, Phys. Rev. Lett. 68, 2512 (1992).

[64] T. R. Morris, Int. J. Mod. Phys. A 9, 2411 (1994).

[65] J. Negele and H. Orland, Quantum Many-Particle Physics (Addison-Wesley, 1988).

[66] J. Nygård, D. H. Cobden, and P. E. Lindelof, Nature 408, 342 (2000).

[67] J. Park, A.N. Pasupathy, J.I. Goldsmith, C. Chang, Y. Yaish, J.R. Petta, M. Rinokoski, J.P. Sethna, H.D. Abruna, and P.L. Mceuen, Nature 417, $722(2002)$.

[68] J.R. Petta, D.C.. Ralph, Phys. Rev. Lett. 87, 266801 (2001).

[69] J. Polchinski, Nucl. Phys. B 231, 269 (1984).

[70] M. Pustilnik and L. Glazman, J. Phys.: Condens. Matter 16, R513 (2004).

[71] D.C. Ralph, R.A. Buhrman, Phys. Rev. Lett. 72, 3401 (1994).

[72] J. Rammer and H. Smith, Rev. Mod. Phys. 58, 323 (1986).

[73] A. Rosch, J. Paaske, J. Kroha, and P. Wölfle, Phys. Rev. Lett. 90, 076804 (2003).

[74] A. Rosch, J. Paaske, J. Kroha, and P. Wölfle, J. Phys. Soc. Jpn. 74, 118 (2005).

[75] M. Salmhofer, Renormalization (Springer, Berlin, 1998).

[76] M. Salmhofer and C. Honerkamp, Prog. Theor. Phys. 105, 1 (2001).

[77] U. Schollwöck, Rev. Mod. Phys. 77, 259 (2005).

[78] H. Schoeller, in Low-Dimensional Systems, edited by T. Brandes (Springer Verlag, 1999), vol. 17, p. 137.

[79] H. Schoeller and J. König, Phys. Rev. Lett. 84, 3686 (2000).

[80] J.H.F. Scott-Thomas and, Phys. Rev. Lett. 62, 583 (1994). 
[81] J.R. Schrieffer and P.A. Wolff, Phys. Rev. 149, 491 (1966).

[82] J. Schwinger J. Math. Phys. 2, 407 (1961).

[83] J. Takahashi and S. Tasaki (2006), cond-mat/0603337.

[84] K. Thygesen and A. Rubio (2006), cond-mat/0609223.

[85] J. von Delft, D.C.. Ralph, Physisics Reports 345, 61 (2001).

[86] F. Wegner, Ann. Physik (Leipzig) 3, 77 (1994).

[87] C. Wetterich, Phys. Lett. B 301, 90 (1993).

[88] K. G. Wilson, Rev. Mod. Phys. 47, 773 (1975).

[89] N. S. Wingreen and Y. Meir, Phys. Rev. B 49, 11040 (1994).

[90] H.R. Zeller, I. Giaever, Phys. Rev. B. 181, 789 (1969). 


\section{Lebenslauf}

Persönliche Angaben:

Geboren am 27 März 1973 In: Rom

Saatsangehörigkeit: italienisch

Berufserfahrung:

2004-2007: Institut für theoretische Physik an der Georg August Universität Göttingen

2002-2004: SIEMENS (Erlangen), Forschungstätigkeit

2000-2002: Forschungstätigkeit bei der Firma ST- Microelectronics Mailand (Italien)

1999-2000: Unix-System Administrator bei der Firma Brionregina s.r.l. Rom (Italien)

1999: Lehrerfahrung in Rom: Anstellung: Lehrer am Gymnasium Leonardo Da Vinci (Rom)

Bildungsweg:

November 2007: Promotion in Physik (Universität Göttingen)

September 1998: Diplom in Physik (Universita' degli studi Roma 3)

September 1987 bis Juli 1992: Besuch des Gymnasiums: Liceo Scientifico Gianbattista Morgagni (Rom)

Juli 1992: Abitur

September 1984 bis Juli 1987: Besuch der Mittelschule (scuola media inferiore): Trilussa (Rom)

September 1979 bis Juni 1984: Besuch der Grundschule: Giorgio Franceschi (Rom) 